\title{
INTEGRAL POINTS ON SYMMETRIC VARIETIES AND SATAKE COMPATIFICATIONS
}

\author{
ALEXANDER GORODNIK, HEE OH AND NIMISH SHAH
}

\begin{abstract}
Let $V$ be an affine symmetric variety defined over $\mathbb{Q}$. We compute the asymptotic distribution of the angular components of the integral points in $V$. This distribution is described by a family of invariant measures concentrated on the Satake boundary of $V$. In the course of the proof, we describe the structure of the Satake compactifications for general affine symmetric varieties and compute the asymptotic of the volumes of norm balls.
\end{abstract}

\section{Contents}

1. Introduction

2. Examples

3. Affine symmetric spaces and representations

4. Structure of Satake compactification

5. Invariant measures at infinity

6. Volume asymptotics

7. Distribution of integral points

8. Comparison with Chambert-Loir-Tschinkel conjecture 45 References

\section{INTRODUCTION}

Let $V=\left\{x \in \mathbb{R}^{n}: f_{1}(x)=\cdots=f_{s}(x)=0\right\}$ with $f_{i} \in \mathbb{Z}\left[x_{1}, \ldots, x_{n}\right]$ be an affine variety. It is a fundamental problem of Diophantine geometry to understand the set of integral points $V(\mathbb{Z})$ in $V$. In particular, when the number of integral points is infinite, one may ask

Question 1.1. Given a norm $\|\cdot\|$ on $\mathbb{R}^{n}$, determine the asymptotic of

$$
N_{T}(V):=\#\{x \in V(\mathbb{Z}):\|x\|<T\}
$$

as $T \rightarrow \infty$.

We are interested in a more refined question:

The first and the second authors partially supported by NSF 0400631 and NSF 0333397, 0629322 respectively. 
Question 1.2. For a radial cone $\mathcal{C}$ in $\mathbb{R}^{n}$ centered at the origin, determine the asymptotic of

$$
N_{T}(V, \mathcal{C}):=\#\{x \in V(\mathbb{Z}) \cap \mathcal{C}:\|x\|<T\}
$$

as $T \rightarrow \infty$.

One can also state an analogous question in terms of convergence of measures. We define a probability measure $\mu_{T}$ on the unit sphere $S^{n-1}$ in $\mathbb{R}^{n}$ :

$$
\mu_{T}:=\frac{1}{N_{T}(V)} \sum_{x \in V(\mathbb{Z}): 0<\|x\|<T} \delta_{\pi(x)},
$$

where $\pi: \mathbb{R}^{n} \backslash\{0\} \rightarrow S^{n-1}$ denotes the radial projection, and $\delta_{z}$ denotes the Dirac measure at $z$. As $T \rightarrow \infty$, these measures characterize the asymptotic distribution of the angular components of points of $V(\mathbb{Z})$.

Question 1.3. Determine the weak ${ }^{*}$ limits of the measures $\mu_{T}$ as $T \rightarrow \infty$.

Recall that a sequence of measures $\left\{\mu_{i}\right\}$ on $S^{n-1}$ converges to $\mu$ in weak* topology if $\int_{S^{n-1}} \phi d \mu_{i} \rightarrow \int_{S^{n-1}} \phi d \mu$ for every $\phi \in C\left(S^{n-1}\right)$.

In this paper we give a complete solution to Questions 1.2 and 1.3 when $V$ is an affine symmetric variety. In this case, Question 1.1 was answered by Duke, Rudnick, Sarnak [DRS] and Eskin, McMullen [EM], though explicit asymptotics in terms of $T$ were not computed in general. Later Eskin, Mozes and Shah developed an approach using the ergodic theory on homogeneous spaces, based on the work of Dani, Margulis ([DM1], DM2]) and Ratner [Ra on unipotent flows.

We note that the method of [EM] shows that $N_{T}(V, \mathcal{C})$ is asymptotic to the volume of the set $\mathcal{C}_{T}:=\{x \in V \cap \mathcal{C}:\|x\|<T\}$, provided the family $\left\{\mathcal{C}_{T}\right\}$ satisfies the so-called well roundedness property. However it is a highly nontrivial task to check whether the sets $\mathcal{C}_{T}$ are well rounded, and this is precisely where the main technical difficulties of this paper lies (see section 1.2 for more discussion on this point).

The above questions 1.2 and 1.3 are motivated by the conjectures of Manin BM, FMT, Peyre [P], and Batyrev, Tschinkel [BT], which describe the distribution of rational points on projective Fano varieties (see Remark 1.13 and [Ts]). Recently, Chambert-Loir and Tschinkel proposed an analogous conjecture for integral points. We expect that our results will support this conjecture (see Section 8 ).

We illustrate our main results by the following example of a quadratic surface. We refer to Section 2 for further examples.

Example 1.4. Fix $n \geq 4$ and $k \in \mathbb{Z} \backslash\{0\}$. Let $Q$ be an integral non-degenerate indefinite quadratic form in $n$ variables such that $Q(x)=k$ has at least one integral solution. Let $\Omega$ be a Borel subset of $S^{n-1}$ such that the interior of $\Omega$ intersects $\{Q=$ $0\}$, and the boundary of $\Omega$ has measure zero with respect to a smooth measure on $\{Q=0\} \cap S^{n-1}$. Then setting $\mathcal{C}=\mathbb{R}^{+} \cdot \Omega$, we have

$$
N_{T}(\{Q=k\}, \mathcal{C}) \sim_{T \rightarrow \infty} \operatorname{Vol}(\{x \in \mathcal{C}: Q(x)=k,\|x\|<T\}) \sim_{T \rightarrow \infty} d_{\mathcal{C}} \cdot T^{n-2},
$$

where the volume is computed with respect to a suitably normalized $\mathrm{SO}(Q)$-invariant measure on $\{Q=k\}$ and $d_{\mathcal{C}}>0$ is a computable constant. 


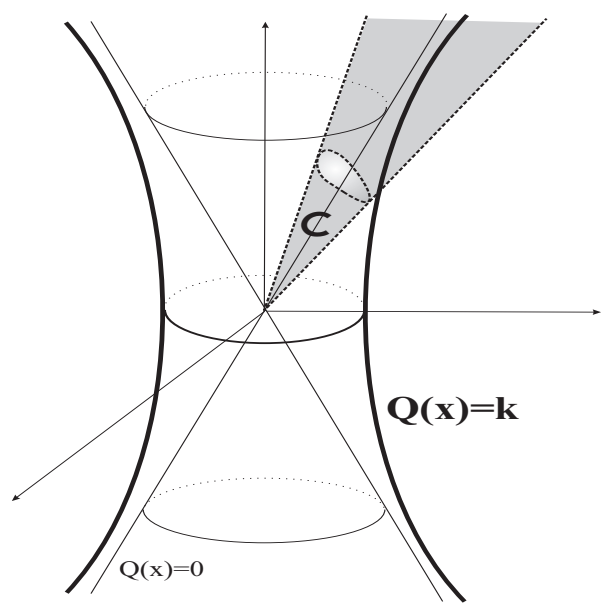

We denote by $\nu$ the unique $\mathrm{SO}(Q)$-invariant measure on $\left\{x \in \mathbb{R}^{n} \backslash\{0\}: Q(x)=0\right\}$ normalized so that

$$
\nu\left(\left\{x \in \mathbb{R}^{n} \backslash\{0\}: Q(x)=0,\|x\|<1\right\}\right)=1 .
$$

Define the measure $\mu$ on $S^{n-1}$ by

$$
\mu:=\pi_{*}\left(\left.\nu\right|_{B_{1}}\right)
$$

where $B_{1}=\left\{x \in \mathbb{R}^{n}:\|x\|<1\right\}$. Then $\mu_{T} \rightarrow \mu$ as $T \rightarrow \infty$.

1.1. Main results. Let $\mathbf{G}$ be a connected $\mathbb{Q}$-simple algebraic $\mathbb{Q}$-group isotropic over $\mathbb{R}$ with a given $\mathbb{R}$-irreducible $\mathbb{Q}$-rational representation $\iota: \mathbf{G} \rightarrow \mathrm{GL}(\mathbf{W})$. Suppose that there exists $v_{0} \in \mathbf{W}(\mathbb{Q})$ such that $\mathbf{G} v_{0}$ is Zariski closed and that $V:=\mathbf{G}(\mathbb{R})^{\circ} v_{0}$ is an affine symmetric (real) variety, that is, the stabilizer $\mathbf{H}$ of $v_{0}$ in $\mathbf{G}$ is the set of fixed point of an involution $\sigma$ of $\mathbf{G}$. Examples of affine symmetric varieties are provided by Proposition 3.16.

Notation. $G=\mathbf{G}(\mathbb{R})^{\circ}, W=\mathbf{W}(\mathbb{R})$, and $H=\mathbf{H}(\mathbb{R}) \cap G$. Then $V \cong G / H$. We assume that $V(\mathbb{Z}) \neq \emptyset$ and that $\mathbf{H}$ has no nontrivial $\mathbb{Q}$-characters.

We fix a basis, say $\mathcal{B}$, of $W$, and define

$$
S(W)=\left\{\sum_{\mathbf{e} \in \mathcal{B}} x_{\mathbf{e}} \mathbf{e} \in W: \sum_{\mathbf{e} \in \mathcal{B}} x_{\mathbf{e}}^{2}=1\right\} .
$$

Let $\pi: W \backslash\{0\} \rightarrow S(W)$ denote the radial projection.

We define the Satake boundary $V^{\infty}$ of $V$ :

$$
V^{\infty}:=\{\lim \pi(v): v \in V, v \rightarrow \infty\}=\overline{\pi(V)}-\pi(V) .
$$

For example, when $V=\left\{x \in \mathbb{R}^{n}: Q(x)=k\right\}$ is a quadratic surface,

$$
V^{\infty}=\left\{x \in S^{n-1}: Q(x)=0\right\} .
$$

The map $\pi$ embeds $V$ homeomorphically into $\overline{\pi(V)}$ as an open dense subset (see Proposition 4.8), and we call $\overline{\pi(V)}$ the Satake compactification of $V$. For a Riemannian symmetric space, this compactification was introduced by Satake in [Sa]. We note that 
Satake [Sa] considered only the special case when $\iota$ is a representation on the space of bi-linear forms.

The action of $G$ on $W$ induces, via $\pi$, a $G$-action on $S(W)$. In Section 4 we will prove that $V^{\infty}$ is a union of finitely many $G$-orbits, which are locally closed.

Given a measure $\mu$ on $V^{\infty}$ which is a linear combination of smooth positive measures on some $G$-orbits, we say that $\mu$ is concentrated on the union of these $G$-orbits.

Let $\mathcal{C}$ be a Borel cone in $W$ centered at the origin. In order to have meaningful results about the sets $V(\mathbb{Z}) \cap \mathcal{C}$, it is necessary to assume that the intersection $V \cap \mathcal{C}$ is large in a suitable sense. As we will see below, the "size" of $V(\mathbb{Z}) \cap \mathcal{C}$ depends quite sensitively on the set of $G$-orbits in $V^{\infty}$ that $\mathcal{C}$ intersects.

A Borel cone $\mathcal{C} \subset W$ centered at 0 is called admissible if the closure of $\mathcal{C}$ has nonempty intersection with $V^{\infty}$, and the boundary of $\mathcal{C}$ is of zero measure with respect to the smooth measure class on each of the finitely many $G$-orbits on $V^{\infty}$. A Borel cone $\mathcal{C} \subset W$ centered at 0 is called generic if the closure of $\mathcal{C}$ and the interior of $\mathcal{C}$ intersect the same collection of $G$-orbits in $V^{\infty}$.

The following theorem gives a natural generalization of Example 1.4. We fix any norm $\|\cdot\|$ on $W$ and set

$$
B_{T}=\{v \in W:\|v\|<T\} .
$$

Theorem 1.6. For every admissible generic cone $\mathcal{C} \subset W$,

$$
\#\left(V(\mathbb{Z}) \cap B_{T} \cap \mathcal{C}\right) \sim_{T \rightarrow \infty} \operatorname{Vol}\left(V \cap B_{T} \cap \mathcal{C}\right) \sim_{T \rightarrow \infty} d_{\mathcal{C}} \cdot T^{a_{\mathcal{C}}}(\log T)^{b_{\mathcal{C}}-1},
$$

where the volume is computed with respect to a suitably normalized $G$-invariant measure on $V$, and $d_{\mathcal{C}}>0, a_{\mathcal{C}} \in \mathbb{Q}^{+}, b_{\mathcal{C}} \in \mathbb{N}$ are computable constants.

Given a Euclidean norm $\|\cdot\|$ on $W$ and $v_{0} \in V^{\infty}$, the cone $\mathcal{C}=\left\{v:\left\|\pi(v)-v_{0}\right\|<\varepsilon\right\}$ is admissible and generic for all sufficiently small $\varepsilon>0$ (see subsection 7.1). Hence, we get the following application of Theorem 1.6 to Diophantine approximation.

Corollary 1.7. Let $\|\cdot\|$ be a Euclidean norm on $W$ and $v_{0} \in V^{\infty}$. Then for any sufficiently small $\varepsilon>0$, there exist $c=c\left(v_{0}, \varepsilon\right)>0, a=a\left(v_{0}\right) \in \mathbb{Q}^{+}, b=b\left(v_{0}\right) \in \mathbb{N}$ such that

$$
\begin{aligned}
\#\left\{v \in V(\mathbb{Z}) \cap B_{T}:\left\|\pi(v)-v_{0}\right\|<\varepsilon\right\} & \sim_{T \rightarrow \infty} \operatorname{Vol}\left(\left\{v \in V \cap B_{T}:\left\|\pi(v)-v_{0}\right\|<\varepsilon\right\}\right) \\
& \sim_{T \rightarrow \infty} c \cdot T^{a}(\log T)^{b-1} .
\end{aligned}
$$

Now we describe the structure of the Satake boundary $V^{\infty}$ of $V$. Let $K$ be a maximal compact subgroup of $G$ compatible with $H$ and $\mathfrak{a}$ a Cartan subalgebra corresponding to the pair $K$ and $H$, so that the Cartan decomposition $G=K \exp (\mathfrak{a}) H$ holds ([Sc, Ch. 7]). We fix a system of simple roots $\Delta_{\sigma}$ of $\mathfrak{a}$ and denote by $\mathfrak{a}^{+} \subset \mathfrak{a}$ the closed positive Weyl chamber. One can choose a subset $\mathcal{W}$ of the normalizer of $\mathfrak{a}$ in $K$ such that we have a decomposition

$$
G=K \exp \left(\mathfrak{a}^{+}\right) \mathcal{W H}
$$

where the $\mathfrak{a}^{+}$-component and the $\mathcal{W}$-component of each element of $G$ are uniquely defined (see Section 3). 
For any subset $J \subset \Delta_{\sigma}$, we set

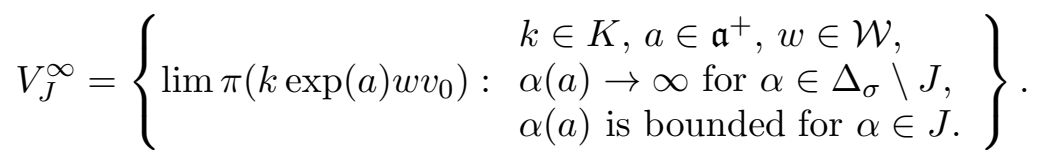

Note that $V_{\Delta_{\sigma}}^{\infty}=\pi(V)$ and

$$
V^{\infty}=\bigcup_{J \subsetneq \Delta_{\sigma}} V_{J}^{\infty}
$$

Every set $V_{J}^{\infty}$ is a union of finitely many $G$-orbits (see Theorem 1.22).

Denoting by $2 \rho$ the sum (with multiplicities) of all positive roots of $\mathfrak{a}$ and by $\lambda_{\iota}$ the highest weight of the representation $\iota$ with respect to $\mathfrak{a}^{+}$(see Section [3.2), we have decompositions

$$
2 \rho:=\sum_{\alpha \in \Delta_{\sigma}} u_{\alpha} \alpha \quad \text { and } \quad \lambda_{\iota}:=\sum_{\alpha \in \Delta_{\sigma}} m_{\alpha} \alpha .
$$

Note that $u_{\alpha}>0, m_{\alpha}>0$, and $u_{\alpha}, m_{\alpha} \in \mathbb{Q}([\mathrm{OV}$, p. 85]). Define

$$
\begin{aligned}
& a_{\iota}=\max \left\{\frac{u_{\alpha}}{m_{\alpha}}: \alpha \in \Delta_{\sigma}\right\}, \\
& I_{\iota}=\left\{\alpha \in \Delta_{\sigma}: \frac{u_{\alpha}}{m_{\alpha}}<a_{\iota}\right\}, \\
& b_{\iota}=\#\left(\Delta_{\sigma} \backslash I_{\iota}\right) \geq 1 .
\end{aligned}
$$

Theorem 1.11. For an admissible cone $\mathcal{C} \subset W$, the limits

$$
\lim _{T \rightarrow \infty} \frac{\#\left(V(\mathbb{Z}) \cap B_{T} \cap \mathcal{C}\right)}{T^{a_{\iota}}(\log T)^{b_{\iota}-1}} \quad \text { and } \quad \lim _{T \rightarrow \infty} \frac{\operatorname{Vol}\left(V \cap B_{T} \cap \mathcal{C}\right)}{T^{a_{\iota}}(\log T)^{b_{\iota}-1}}
$$

exist and are equal, where the volume is computed with respect to a suitably normalized $G$-invariant measure on $V$. Moreover, if $\mathcal{C}^{\circ} \cap V_{I_{\iota}}^{\infty} \neq \emptyset$, then the limits are strictly positive.

We also extend the result about convergence of measures in Example 1.4 to general affine symmetric spaces. There is an explicitly given $G$-invariant measure $\nu_{\iota}$ on $\mathbb{R}^{+} \cdot V_{I_{\iota}}^{\infty}$, normalized such that $\nu_{\iota}\left(B_{1}\right)=1$. The measure $\nu_{\iota}$ is homogeneous of degree $a_{\iota}$, and we have a decomposition

$$
d \nu_{\iota}(t \cdot \theta)=t^{a_{\iota}-1} d t d \theta, \quad t \in \mathbb{R}^{+}, \theta \in V_{I_{\iota}}^{\infty},
$$

where $d t$ is a Lebesgue measure on $\mathbb{R}^{+}$and $d \theta$ is a smooth measure on $V_{I_{\iota}}^{\infty}$. We define the probability measure $\mu_{\iota}$ on $V_{I_{\iota}}^{\infty}$ by $\mu_{\iota}=\pi_{*}\left(\left.\nu_{\iota}\right|_{B_{1}}\right)$ or equivalently,

$$
d \mu_{\iota}(\theta)=\|\theta\|^{-a_{\iota}} d \theta, \quad \theta \in V_{I_{\iota}}^{\infty} .
$$

Note that the norm $\|\cdot\|$ need not be constant on $S(W)$ for our fixed choice of the sphere $S(W)$. Later on, we give an explicit formula for $\mu_{\iota}$ (see (7.17)).

Theorem 1.12. As $T \rightarrow \infty$, we have

$$
\mu_{T} \rightarrow \mu_{\iota}
$$


Theorem 1.12 also holds for representations $\iota$ which are not irreducible (see Remark 6.11).

In the case of the group variety (i.e., when $G=L \times L$ and $H=\{(l, l): l \in L\}$ ), these equidistribution results (Theorem 1.11 and Theorem 1.12) were first proved by Maucourant [M], although not in terms of the Satake boundary.

Remark 1.13. It is interesting to compare Theorem 1.12 with a result in GMO (see also [STT]), which describes distribution of rational points of bounded height. Let $\mathbf{G}$ be a connected adjoint absolutely simple algebraic $\mathbb{Q}$-group and $\iota: \mathbf{G} \rightarrow \mathrm{GL}_{N}$ an absolutely irreducible representation defined over $\mathbb{Q}$. We denote by $\bar{\iota}$ the corresponding map from $\mathbf{G}$ to the projective space $\mathbb{P}^{N^{2}-1}$ and by $\mathrm{H}=\mathrm{H}_{\infty} \cdot \prod_{p \text { :prime }} \mathrm{H}_{p}$ a height function on $\mathbb{P}^{N^{2}-1}(\mathbb{Q})$ where $\mathrm{H}_{\infty}$ is a norm on $\mathbb{R}^{N^{2}}$. Let $G=\mathbf{G}(\mathbb{R})^{\circ}, G(\mathbb{Q})=G \cap \mathbf{G}(\mathbb{Q})$ and $G(\mathbb{Z})=G \cap \mathbf{G}(\mathbb{Z})$. As explained in Section 2.4. $V=\iota(G)$ is an affine symmetric variety, and we have a decomposition

$$
\overline{\bar{\imath}(G)}=\bigcup_{I \subset \Delta_{\sigma}} V_{I}^{\infty}
$$

It follows from [GMO] that for every $\phi \in C(\overline{\bar{\iota}(G)})$,

$$
\lim _{T \rightarrow \infty} \frac{1}{\#\{\gamma \in G(\mathbb{Q}): \mathrm{H}(\bar{\iota}(\gamma))<T\}} \sum_{\gamma \in G(\mathbb{Q}): \mathrm{H}(\bar{\iota}(\gamma))<T} \phi(\bar{\iota}(\gamma))=\int_{V_{\Delta_{\sigma}}^{\infty}} \phi(\omega) \frac{d \omega}{\mathrm{H}_{\infty}(\omega)^{a_{\iota}^{\prime}}}
$$

where $d \omega$ is a $G$-invariant measure on $V_{\Delta_{\sigma}}^{\infty}=\bar{\iota}(G)$ and $a_{\iota}^{\prime}>a_{\iota}$. On the other hand, it follows from Theorem 1.12 that for every $\phi \in C(\overline{\bar{l}(G)})$,

$$
\lim _{T \rightarrow \infty} \frac{1}{\#\left\{\gamma \in G(\mathbb{Z}): \mathrm{H}_{\infty}(\bar{\iota}(\gamma))<T\right\}} \sum_{\gamma \in G(\mathbb{Z}): \mathrm{H}_{\infty}(\bar{\iota}(\gamma))<T} \phi(\bar{\iota}(\gamma))=\int_{V_{I_{\iota}}^{\infty}} \phi(\theta) \frac{d \theta}{\mathrm{H}_{\infty}(\theta)^{a_{\iota}}}
$$

Note that for affine symmetric varieties of higher rank, $\mu_{\iota}=\lim _{T \rightarrow \infty} \mu_{T}$ is concentrated on $V_{I_{L}}^{\infty}$, which might have empty interior in $V^{\infty}$ (see Section 2 for examples). In particular, Theorem 1.12 does not imply Theorem 1.6. To prove Theorem 1.6, we need to investigate the accumulation of integral points on all components of $V^{\infty}$.

Definition 1.14. A subset $I \subset \Delta_{\sigma}$ is called $\lambda_{\iota}$-connected if the Dynkin diagram of $\left\{\lambda_{\iota}\right\} \cup I$ is connected. In other words, if $I \cup\left\{\lambda_{\iota}\right\}=S_{1} \cup S_{2}, S_{i} \neq \emptyset$, then $S_{1} \not \perp S_{2}$ with respect to the identification of $\mathfrak{a}^{*}$ with $\mathfrak{a}$ via the Killing form.

We show (see Theorem 1.22) that

$$
V^{\infty}=\bigsqcup_{\lambda_{\iota} \text {-connected } I \subsetneq \Delta_{\sigma}} V_{I}^{\infty},
$$

and for $\lambda_{\iota}$-connected $I, J \subset \Delta_{\sigma}$,

$$
I \subsetneq J \Longleftrightarrow V_{I}^{\infty} \subset \partial\left(V_{J}^{\infty}\right) .
$$


For $\lambda_{\iota}$-connected $I \subsetneq \Delta_{\sigma}$, we set

$$
\begin{aligned}
& a_{\iota}(I):=\max \left\{\frac{u_{\alpha}}{m_{\alpha}}: \alpha \in \Delta_{\sigma} \backslash I\right\}>0, \\
& I_{\iota}(I):=I \cup\left\{\alpha \in \Delta_{\sigma} \backslash I: \frac{u_{\alpha}}{m_{\alpha}}<a_{\iota}(I)\right\} \subsetneq \Delta_{\sigma}, \\
& b_{\iota}(I):=\#\left(\Delta_{\sigma} \backslash I_{\iota}(I)\right) \geq 1 .
\end{aligned}
$$

Note that $I_{\iota}(\emptyset)=I_{\iota}$. We will show in Proposition 5.12 that $I_{\iota}(I)$ is $\lambda_{\iota}$-connected. We consider the lexicographical order on the set of pairs $(a, b) \in \mathbb{R} \times \mathbb{R}$. Note that for $\lambda_{\iota}$-connected subsets $I$ and $J$ of $\Delta_{\sigma}$,

$$
I \subset J \Longrightarrow I_{\iota}(I) \subset I_{\iota}(J) \Longrightarrow\left(a_{\iota}(I), b_{\iota}(I)\right) \geq\left(a_{\iota}(J), b_{\iota}(J)\right) .
$$

For $\Omega \subset W$ with $\overline{\pi(\Omega \backslash\{0\})} \cap V^{\infty} \neq \emptyset$, we define

$$
\begin{aligned}
\Theta_{\Omega} & :=\left\{I \subsetneq \Delta_{\sigma}: I \text { is } \lambda_{\iota} \text {-connected and } \overline{\pi(\Omega \backslash\{0\})} \cap V_{I}^{\infty} \neq \emptyset\right\}, \\
\left(a_{\iota}(\Omega), b_{\iota}(\Omega)\right) & :=\max \left\{\left(a_{\iota}(I), b_{\iota}(I)\right): I \in \Theta_{\Omega}\right\}, \\
\Theta_{\iota}(\Omega) & :=\left\{I_{\iota}(I): I \in \Theta_{\Omega},\left(a_{\iota}(I), b_{\iota}(I)\right)=\left(a_{\iota}(\Omega), b_{\iota}(\Omega)\right)\right\} \subset \Theta_{\Omega} .
\end{aligned}
$$

Roughly speaking, we show that the asymptotic number of points in $V(\mathbb{Z})$, with norm less than $T$, whose images under $\pi$ accumulate on $\Omega$, is of the order $T^{a_{\iota}(\Omega)}(\log T)^{b_{\iota}(\Omega)-1}$.

It might happen that in Theorem 1.11, both of the limits are zero. This simply means that the normalization term $T^{a_{\iota}}(\log T)^{b_{\iota}-1}$ is not suitable. We prove a refined version of Theorem 1.11.

Theorem 1.18. For every admissible cone $\mathcal{C} \subset W$, the limits

$$
\lim _{T \rightarrow \infty} \frac{\#\left(V(\mathbb{Z}) \cap B_{T} \cap \mathcal{C}\right)}{T^{a_{\iota}(\mathcal{C})}(\log T)^{b_{\iota}(\mathcal{C})-1}} \quad \text { and } \quad \lim _{T \rightarrow \infty} \frac{\operatorname{Vol}\left(V \cap B_{T} \cap \mathcal{C}\right)}{T^{a_{\iota}(\mathcal{C})}(\log T)^{b_{\iota}(\mathcal{C})-1}}
$$

exist and are equal, where the volume is computed with respect to a suitably normalized $G$-invariant measure on $V$. Moreover, if $\mathcal{C}^{\circ} \cap V_{I}^{\infty} \neq \emptyset$ for some $I \in \Theta_{\iota}(\mathcal{C})$, then the limits are strictly positive.

Moreover if $\mathcal{C}$ is generic, then for any $I \in \Theta_{\mathcal{C}}, \mathcal{C}^{\circ} \cap V_{I}^{\infty} \neq \emptyset$; and by (1.15), we get $\mathcal{C}^{\circ} \cap V_{I_{\iota}(I)}^{\infty} \neq \emptyset$. Therefore Theorem 1.18 implies Theorem 1.6.

Let $\mathcal{D}_{\iota}=\left\{I_{\iota}(I): \lambda_{\iota}\right.$-connected $\left.I \subsetneq \Delta_{\sigma}\right\}$. To state the next result, we will introduce a family of smooth measures $\mu_{I}$ on $V_{I}^{\infty}$ for $I \in \mathcal{D}_{\iota}$. For each $I \in \mathcal{D}_{\iota}$, here is an explicitly given $G$-invariant measure $\nu_{I}$ on $\mathbb{R}^{+} \cdot V_{I}^{\infty}$, which is a finite union of $G$-orbits. Note that $a_{I}=\frac{u_{\alpha}}{m_{\alpha}}$ is constant for $\alpha \in \Delta_{\sigma} \backslash I$, and the measure $\nu_{I}$ is homogeneous of degree $a_{I}$. We have a decomposition

$$
d \nu_{I}(t \cdot \theta)=t^{a_{I}-1} d t d \theta, \quad t \in \mathbb{R}^{+}, \theta \in V_{I}^{\infty},
$$

where $d t$ is a Lebesgue measure on $\mathbb{R}$ and $d \theta$ is a smooth measure on $V_{I}^{\infty}$. We define $\mu_{I}=\pi_{*}\left(\left.\nu_{I}\right|_{B_{1}}\right)$ or equivalently,

$$
d \mu_{I}(\theta)=\|\theta\|^{-a_{I}} d \theta, \quad \theta \in V_{I}^{\infty} .
$$


An explicit formula for $\mu_{I}$ is given in (7.18). For $\mathcal{I} \subset \mathcal{D}_{\iota}$, we define

$$
\mu_{\mathcal{I}}=\sum_{I \in \mathcal{I}} \mu_{I} \quad \text { and } \quad \nu_{\mathcal{I}}=\sum_{I \in \mathcal{I}} \nu_{I}
$$

Theorem 1.19. For every $\phi \in C(S(W))$ with $\operatorname{supp} \phi \cap V^{\infty} \neq \emptyset$, we have

$$
\lim _{T \rightarrow \infty} \frac{1}{T^{a_{\iota}(\phi)}(\log T)^{b_{\iota}(\phi)-1}} \sum_{x \in V(\mathbb{Z}): 0<\|x\|<T} \phi(\pi(x))=c \int_{S(W)} \phi d \mu_{\Theta_{\iota}(\phi)},
$$

where $c>0$ depends only on $V(\mathbb{Z})$, and $a_{\iota}(\phi)=a_{\iota}(\operatorname{supp} \phi), b_{\iota}(\phi)=b_{\iota}(\operatorname{supp} \phi), \Theta_{\iota}(\phi)=$ $\Theta_{\iota}(\operatorname{supp} \phi)$.

Note that if $\operatorname{supp} \phi \cap V^{\infty} \neq \emptyset$, then $\phi(\pi(x))=0$ for all but finitely many $x \in V(\mathbb{Z})$.

The measures $\mu_{\mathcal{I}}$ are analogues of the Patterson-Sullivan measures, which are concentrated on the visual boundary of a Riemannian symmetric space.

In order to prove Theorems 1.18 and 1.19, we compare the asymptotic distribution of integral points to the corresponding continuous asymptotic distribution, which is given in the following theorem.

Theorem 1.20. For every $f \in C_{c}(W \backslash\{0\})$ with $\pi(\operatorname{supp} f) \cap V^{\infty} \neq \emptyset$,

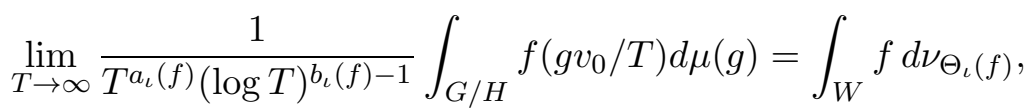

where $a_{\iota}(f)=a_{\iota}(\pi(\operatorname{supp} f)), b_{\iota}(f)=b_{\iota}(\pi(\operatorname{supp} f))$, and $\Theta_{\iota}(f)=\Theta_{\iota}(\pi(\operatorname{supp} f))$.

Note that the limit measure $\nu_{\Theta_{\iota}(f)}$ is homogeneous of degree $a_{\iota}(f)$.

Also note that if $f \in C_{c}(W \backslash\{0\})$ and $\pi(\operatorname{supp} f) \cap V^{\infty}=\emptyset$, then for all large $T$, $f\left(g v_{0} / T\right)=0$ for all $g \in G$.

Remark 1.21. The condition that the group $\mathbf{G}$ is $\mathbb{Q}$-simple can be relaxed. In fact, it suffices to assume that every $\mathbb{Q}$-simple factor $\mathbf{G}_{0}$ of $\mathbf{G}$ is isotropic over $\mathbb{R}$ and $\mathbf{G}=\mathbf{G}_{0} \mathbf{H}$. A small modification in the proof is required only in Section 7 (see Remark 7.2).

1.2. Ingredients of the proof. A common strategy for estimating the number of integral points in various domains involves two steps:

(1) establishing suitable regularity of domains and their volumes;

(2) comparing the number of integral points with the volumes of the domains.

The second step, discussed in Section 7 , is essentially done using standard techniques developed in [DRS, EM] in view of the equidistribution theorem (Theorem 7.1) available in the symmetric setting. Checking the first step for the domains defined by the intersection of a cone with the norm balls contains the main technical difficulties of the paper and requires, in particular, the analysis of the structure of the Satake boundary (Section 4) and asymptotic estimates for renormalized volumes with respect to the invariant measure (Sections 5 and (6). For instance, one of the reasons we are working in the setting of a symmetric space, rather than of a general homogeneous space, is the lack of the structure theory for Satake compactification needed to establish (1), since Theorem 7.1 is available in a more general setting of homogeneous spaces as obtained in [EMS. 
We mention that checking the well-roundedness property of [EM] for the domains amounts to carrying out the first step. Setting, for a radial cone $\mathcal{C}$ and $T>0$,

$$
\mathcal{C}_{T}:=\{x \in V \cap \mathcal{C}:\|x\|<T\},
$$

$\left\{\mathcal{C}_{T}: T \gg 1\right\}$ is in general not well-rounded. We introduce the notion of an admissible generic cone in terms of its intersection with the Satake boundary of $V$. Showing that the family $\left\{\mathcal{C}_{T}: T \gg 1\right\}$ is well-rounded for an admissible generic cone $\mathcal{C}$ is a consequence of two main ingredients of the paper:

(i) Tube Lemma (Coro 1.23); in showing this lemma, we needed to generalize Satake's theory in an affine symmetric setting.

(ii) The computation of the asymptotic limit of the invariant measures on $V$ (Theorem 1.20).

We emphasize that in order to compute the explicit volume asymptotic of $\mathcal{C}_{T}$, we need only the part (ii). However in order to obtain that $N_{T}(V, \mathcal{C}) \sim \operatorname{Vol}\left(\mathcal{C}_{T}\right)$ (not to mention the explicit asymptotic), we need to use both (i) and (ii).

In the rest of this section, we explain the generalization of Satake's result [Sa] on Riemannian symmetric spaces, which summarizes properties of the decomposition (1.8) and Tube lemma.

Theorem 1.22. (a) For every $J \subset \Delta_{\sigma}, V_{J}^{\infty}$ is a union of finitely many $G$-orbits.

(b) For every $J \subset \Delta_{\sigma}$,

$$
\overline{V_{J}^{\infty}}=\bigcup_{I \subset J} V_{I}^{\infty} .
$$

(c) For every $J \subset \Delta_{\sigma}, V_{J}^{\infty}=V_{I}^{\infty}$ where $I$ is the largest $\lambda_{\iota}$-connected subset of $J$.

(d) For distinct $\lambda_{\iota}$-connected subsets $I, J \subset \Delta_{\sigma}, V_{I}^{\infty} \cap V_{J}^{\infty}=\emptyset$.

Similar constructions can be also found in $[\mathrm{Sa}], \mathrm{Os}]$, and $[\mathrm{CP}]$, but they are not suitable for our purpose: Satake [Sa] considered only representations on the space of bilinear forms, the Oshima compactification [Os] is defined abstractly, and the de ConciniProcesi compactification [CP] is defined with respect to the Zariski topology and applies only to a specific type of representations which have regular highest weights.

While Theorem 1.22 is not used in the proofs of Theorem 1.11 and Theorem 1.12 , it is essential for the proofs of Theorem 1.6, Theorem 1.18, and Theorem 1.19, The crucial observation is the following corollary that describes neighborhoods of subsets in $V^{\infty}$. For $I \subset \Delta_{\sigma}$, we set

$$
\mathfrak{a}_{I}=\operatorname{ker} I=\{a \in \mathfrak{a}: \alpha(a)=0, \forall \alpha \in I\},
$$

and $\mathfrak{a}_{I}^{+}=\mathfrak{a}_{I} \cap \mathfrak{a}^{+}$, which is a face of the closed Weyl chamber $\mathfrak{a}^{+}$.

Corollary 1.23 (Tube lemma). Let $I$ be a $\lambda_{\iota}$-connected subset of $\Delta_{\sigma}$ and $\Omega$ a compact subset of $S(W)$ contained in $\bigcup_{J \supset I} V_{J}^{\infty}$. Then there exists a compact set $U \subset \mathfrak{a}^{+}$such that

$$
\Omega \cap \pi(V) \subset \pi\left(K \exp \left(U+\mathfrak{a}_{I}^{+}\right) \mathcal{W} v_{0}\right)
$$


Note that by Theorem $1.22(\mathrm{~b}), \bigcup_{J \supset I} V_{J}^{\infty}$ is the smallest open subset of $\overline{\pi(V)}=$ $V^{\infty} \cup \pi(V)$ which contains $V_{I}^{\infty}$ and is a union of cells. The following picture illustrates the structure of the Satake boundary for an affine symmetric variety associated to an $\mathbb{R}$-irreducible representation of $\mathrm{SL}_{3}$ (see Section 2.4) with the highest weight $\lambda_{\iota}$ which is not orthogonal to the simple roots $\alpha$ and $\beta$. The shaded regions correspond to neighborhoods of points in the components of $V^{\infty}$.

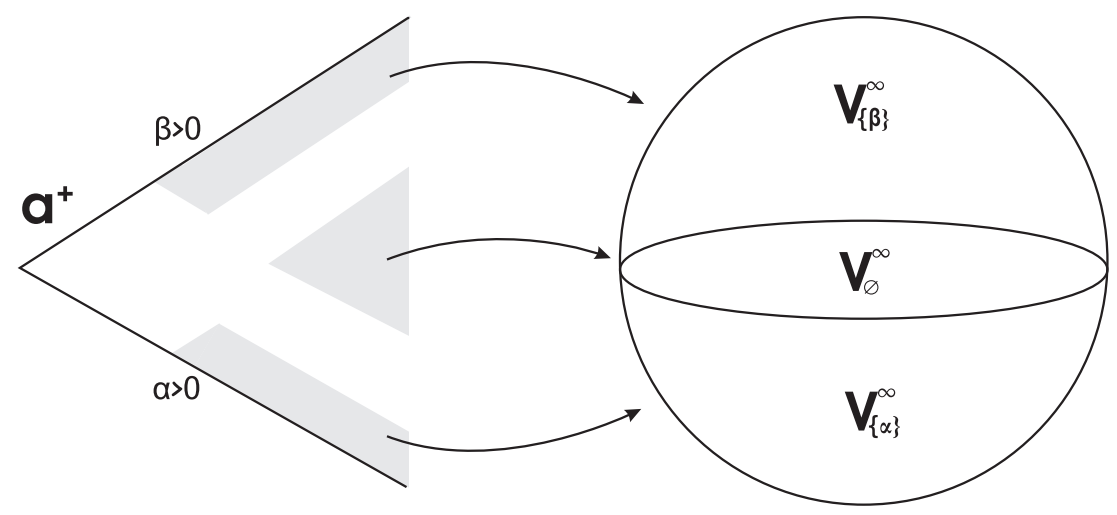

On the other hand, if $\lambda_{\iota} \perp \alpha$, we get

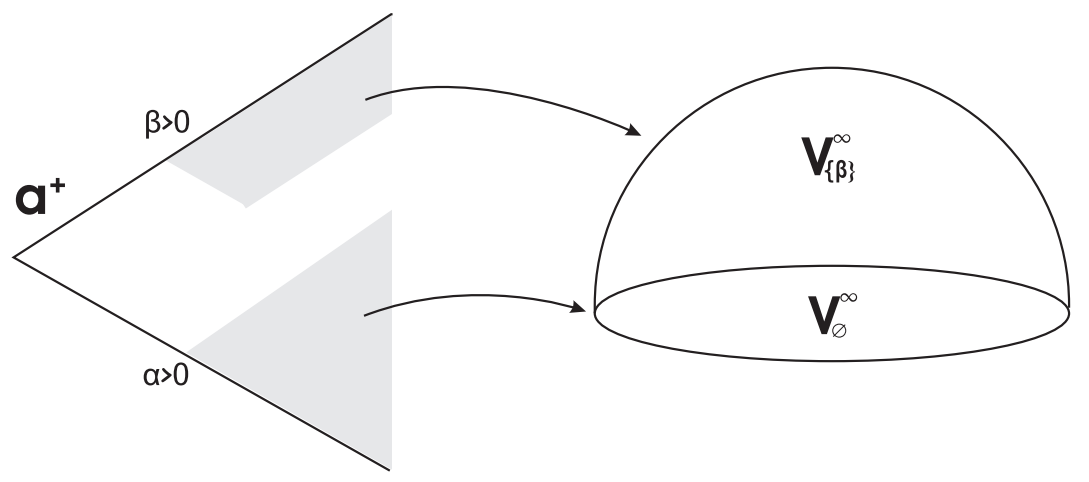

As in the earlier works on counting integral points [DRS, EM], the basic dynamical or ergodic theoretic ingredient in our proof is the result on limiting distributions of translates of closed $H$-orbits in $G / G(\mathbb{Z})$ as established in [EM] (see Theorem [7.1).

1.3. Organization of the paper. Section 2 contains examples. In Section 3 , we review some basic properties of affine symmetric spaces and representation. The structure of the Satake boundary, including the proofs of Theorem 1.22 and Corollary 1.23, is discussed in Section 4. Explicit formula for the measures $\nu_{I}$ are obtained in Section 5 , Section 6 contains results on volume asymptotics (Theorem 1.20), which are described via $G$-invariant measures on the boundary. Finally, the main theorems are proved in Section 7. In Section 8, we state a version of our main result using the language of arithmetic geometry.

1.4. Acknowledgment. The authors would like to thank Gopal Prasad for providing us some important arguments used in the proof of Proposition 4.4 . 


\section{EXAMPLES}

2.1. Quadric. We start with an example of a rank-one symmetric space where the structure of the Satake boundary is quite simple. Let $Q$ be an integral non-degenerate quadratic form on $\mathbb{R}^{p+q}$ of signature $(p, q), p, q \geq 1, p+q \geq 4$, and $k \in \mathbb{N}$. We are interested in the distribution of integral points lying on the the quadratic surface $V:=\left\{Q=k^{2}\right\}$. To simplify notation, we assume that

$$
Q\left(x_{1}, \ldots, x_{p+q}\right)=x_{1}^{2}+\cdots+x_{p}^{2}-x_{p+1}^{2}-\cdots-x_{p+q}^{2}
$$

Let $v_{0}=(k, 0, \ldots, 0) \in V$ and $H=\operatorname{Stab}_{G}\left(v_{0}\right)$. Note that $H$ is the set of fixed points of the involution of $G$ :

$$
\sigma: g \mapsto \operatorname{diag}(-1,1, \ldots, 1) \cdot g \cdot \operatorname{diag}(-1,1, \ldots, 1),
$$

which commutes with the Cartan involution $\theta(g)={ }^{t} g^{-1}$. We have the Cartan decomposition $G=K \exp \left(\mathfrak{a}^{+}\right) \mathcal{W H}$ where

$$
K=\mathrm{SO}(p) \times \mathrm{SO}(q), \quad \mathfrak{a}^{+}=\mathbb{R}_{\geq 0} \cdot\left(E_{p+q, 1}+E_{1, p+q}\right),
$$

and $\mathcal{W}=\{e\}$ if $q>1$ and $\mathcal{W}=\operatorname{diag}( \pm 1,1, \ldots, 1)$ if $q=1$. (Here $E_{i j}$ is the matrix with 1 at $(i, j)$-entry and 0 at the other entries.) For $p>1, V=G v_{0}$ and for $p=1$, $V=G v_{0} \cup G\left(-v_{0}\right)$. Setting $v_{0}^{\infty}=(1 / \sqrt{2}, 0, \ldots, 0,1 / \sqrt{2})$, we have

$$
V^{\infty}= \begin{cases}K v_{0}^{\infty} & \text { for } p, q>1, \\ K v_{0}^{\infty} \sqcup-K v_{0}^{\infty} & \text { otherwise. }\end{cases}
$$

Note that in all cases, $V^{\infty} \simeq S^{p-1} \times S^{q-1}$, where we set $S^{0}=\{ \pm 1\}$. One can check that $a_{\iota}=p+q-2$ and $b_{\iota}=1$. If $V(\mathbb{Z}) \neq \emptyset$, the limiting distribution of the points $\{\pi(v): v \in V(\mathbb{Z}),\|v\|<T\}$ as $T \rightarrow \infty$ is given by the probability measure $\frac{d v}{\|v\|^{p+q-2}}$ where $d v$ is the suitably normalized invariant measure on $S^{p-1} \times S^{q-1}$ (see (7.17)); note that $\|\cdot\|$ can be any given norm on $\mathbb{R}^{p+q}$.

2.2. Determinant surface. For $k \in \mathbb{Z} \backslash\{0\}$, let

$$
V=\{v \in \mathrm{M}(n, \mathbb{R}): \operatorname{det}(v)=k\} .
$$

Fix $v_{0} \in V$. Note that $V$ is a homogeneous space of $G=\mathrm{SL}(n, \mathbb{R}) \times \mathrm{SL}(n, \mathbb{R})$ for the action

$$
\left(g_{1}, g_{2}\right) \cdot v \mapsto g_{1} v\left(v_{0}^{-1} g_{2}^{-1} v_{0}\right), \quad\left(g_{1}, g_{2}\right) \in G, v \in V,
$$

and $H=\operatorname{Stab}_{G}\left(v_{0}\right)$ is the diagonal embedding of $\operatorname{SL}_{n}(\mathbb{R})$ in $G$, which is the fixed point set of the involution $\sigma\left(g_{1}, g_{2}\right)=\left(g_{2}, g_{1}\right)$ commuting with the standard Cartan involution. We have Cartan decomposition $G=K \exp \left(\mathfrak{a}^{+}\right) H$ (note that $\mathcal{W}=\{e\}$ ), where

$$
\begin{aligned}
K & =\mathrm{SO}(n) \times \mathrm{SO}(n), \\
\mathfrak{a}^{+} & =\left\{(a,-a): a=\operatorname{diag}\left(s_{1}, \ldots, s_{n}\right), \sum_{i} s_{i}=0, s_{i}-s_{j} \geq 0 \text { if } i<j\right\} .
\end{aligned}
$$

The simple roots are $\alpha_{i}=s_{i}-s_{i+1}, i=1, \ldots, n-1$, the fundamental weights are $\omega_{i}=\sum_{j=1}^{i} s_{i}$, and $i=1, \ldots, n-1$, and the highest weight is $\lambda_{\iota}=2 \omega_{1}$. Hence, 
the $\lambda_{\iota}$-connected subsets of the set of simple roots are $I_{0}=\emptyset$ and $I_{j}=\left\{\alpha_{1}, \ldots, \alpha_{j}\right\}$, $j=1, \ldots, n-1$. We have

$$
\begin{aligned}
& V^{\infty}=\{v \in S(\mathrm{M}(n, \mathbb{R})): \operatorname{det}(v)=0\}, \\
& V_{I_{j}}^{\infty}=\{v \in S(\mathrm{M}(n, \mathbb{R})): \operatorname{rank}(v)=j+1\} .
\end{aligned}
$$

Since

$$
2 \rho=2 \sum_{j} j(n-j) \alpha_{j} \quad \text { and } \quad \lambda_{\iota}=2 \omega_{1}=2 \sum_{j} \frac{n-j}{n} \alpha_{j},
$$

we have $a_{\iota}=n^{2}-n, b_{\iota}=1, I_{\iota}=I_{n-2}$. Hence, the results from Section 1 (see Remark 1.21) imply that for any admissible cone $\mathcal{C} \subset \mathrm{M}(n, \mathbb{R})$ that contains a degenerate matrix in its interior,

$$
\#\{v \in \mathrm{M}(n, \mathbb{Z}) \cap \mathcal{C}: \operatorname{det}(v)=k,\|v\|<T\} \sim_{T \rightarrow \infty} c(\mathcal{C}, k) \cdot T^{n^{2}-n},
$$

where $c(\mathcal{C}, k)>0$, and the measures

$$
T^{-\left(n^{2}-n\right)} \sum_{v \in V(\mathbb{Z}):\|v\|<T} \delta_{\pi(v)}
$$

converge as $T \rightarrow \infty$ to a finite smooth measure concentrated on the set of matrices of rank $n-1$ in $S(\mathrm{M}(n, \mathbb{R}))$.

2.3. Space of symmetric matrices. Let $V$ be the space of real symmetric matrices of signature $(p, q)$ of determinant $(-1)^{q}$. Put $J=\operatorname{diag}(\underbrace{1, \ldots, 1}_{p}, \underbrace{-1, \ldots,-1}_{q}) \in V$. Then

$$
V=\left\{g J^{t} g: g \in \mathrm{SL}(p+q, \mathbb{R})\right\} \simeq \mathrm{SL}(p+q, \mathbb{R}) / \mathrm{SO}(p, q) .
$$

Let $n=p+q, G=\mathrm{SL}(n, \mathbb{R})$ and $H=\mathrm{SO}(p, q)$. Note that $V$ is the orbit of $J$ for the representation $\iota$ of $G$ on the space $W$ of symmetric $n \times n$ matrices given by

$$
g \cdot w \mapsto g w^{t} g, \quad g \in G, w \in W .
$$

Also, $H$ is the the set of fixed points of the involution $\sigma: g \mapsto J^{t} g^{-1} J$, which commutes with the Cartan involution $\theta: g \mapsto{ }^{t} g^{-1}$. We have Cartan decomposition $G=K \exp \left(\mathfrak{a}^{+}\right) \mathcal{W} H$ where $K=\mathrm{SO}(n), \mathfrak{a}^{+}$is the standard Weyl chamber in $G$, and $\mathcal{W}$ is the subset of the monomial matrices which gives coset representatives for

$$
N_{K}(\mathfrak{a}) / N_{K \cap H}(\mathfrak{a}) Z_{K}(\mathfrak{a}) \simeq S_{n} /\left(S_{p} \times S_{q}\right)
$$

where $S_{n}$ denotes the group of symmetries on $n$ elements. The simple roots $\alpha_{i}$ and the fundamental weights $\omega_{i}$ are defined as in Section [2.2, and the highest weight is given by $\lambda_{\iota}=2 \omega_{1}$. In particular, it follows that the $\lambda_{\iota}$-connected sets are $I_{0}=\emptyset$, $I_{j}=\left\{\alpha_{1}, \ldots, \alpha_{j}\right\}, j=1, \ldots, n-1$, and we have

$$
\begin{aligned}
& V_{I_{j}}^{\infty}=\{v \in S(W): \operatorname{sign}(v)=(r, s), r+s=j, r \leq p, s \leq q\}, \\
& V^{\infty}=\{v \in S(W): \operatorname{sign}(v)=(r, s), r+s<n, r \leq p, s \leq q\} .
\end{aligned}
$$

Note that in this case, the sets $V_{I_{j}}^{\infty}$ are unions of several orbits of $G$ if $p, q>0$. For example, $V_{I_{n-1}}^{\infty}$ is a union of two open orbits which consist of matrices of signature $(p-1, q)$ and $(p, q-1)$ respectively. One can check (as in Section 2.2) that $a_{\iota}=$ 
$\left(n^{2}-n\right) / 2, b_{\iota}=1, I_{\iota}=I_{n-2}$. Hence, the results of Section 1 imply that for every admissible cone $\mathcal{C} \subset W$ which contains a degenerate symmetric matrix in its interior,

$$
\#\{r \in V(\mathbb{Z}):\|r\|<T\} \sim_{T \rightarrow \infty} c(\mathcal{C}) \cdot T^{\frac{n^{2}-n}{2}},
$$

where $c(\mathcal{C})>0$, and the measures

$$
T^{-\frac{n^{2}-n}{2}} \sum_{v \in V(\mathbb{Z}): 0<\|v\|<T} \delta_{\pi(v)}
$$

converge as $T \rightarrow \infty$ to a measure concentrated on the set of matrices of signature $(p-1, q)$ and $(p, q-1)$ in $S(W)$.

2.4. Group variety. Let $\mathbf{G}$ be a connected $\mathbb{Q}$-simple algebraic group isotropic over $\mathbb{R}$ and $\iota: \mathbf{G} \rightarrow \mathrm{GL}(W)$ an $\mathbb{R}$-irreducible $\mathbb{Q}$-representation of $\mathbf{G}$. We consider the distribution of integral points in the variety $\mathbf{V}:=\iota(\mathbf{G})$. Note that $\mathbf{V}(\mathbb{R})$ consists of finitely many orbits of $G=\mathbf{G}(\mathbb{R})^{\circ}$. For simplicity, we make the computation for the orbit $V=\iota(G)$.

Let $K$ be a maximal compact subgroup of $G, \mathfrak{a}$ a Cartan subalgebra associated to $K$, and $\mathfrak{a}^{+}$a positive Weyl chamber. We denote by $\Delta$ the set of simple roots of $G$ with respect to $\mathfrak{a}^{+}$, and let $\omega_{\alpha}, \alpha \in \Delta$, be the set of fundamental weights. We consider the action of $\tilde{G}=G \times G$ on $V$ :

$$
\left(g_{1}, g_{2}\right) \cdot v \mapsto g_{1} v g_{2}^{-1}, \quad\left(g_{1}, g_{2}\right) \in \tilde{G}, v \in V .
$$

Then $V \simeq \tilde{G} / H$, where $H=\{(g, g): g \in G\}$. We have Cartan decomposition $\tilde{G}=$ $\tilde{K} \exp \left(\tilde{\mathfrak{a}}^{+}\right) H$ (note that $\mathcal{W}=\{e\}$ ), where

$$
\tilde{K}=K \times K \quad \text { and } \quad \tilde{\mathfrak{a}}^{+}=\left\{(a,-a): a \in \mathfrak{a}^{+}\right\} .
$$

Note that in this case every $V_{I}^{\infty}$ is a single $G$-orbit. Let $\rho$ and $\tilde{\rho}$ be half of the sums of positive roots for $G$ and $\tilde{G}$, and $\lambda_{\iota}$ and $\tilde{\lambda}_{\iota}$ be the highest weights for $\mathfrak{a}$ and $\tilde{\mathfrak{a}}$ respectively. Since $\tilde{\rho}=2 \rho$ and $\tilde{\lambda}_{\iota}=2 \lambda_{\iota}$, the parameters $a_{\iota}, b_{\iota}, I_{\iota}$ are computed as in (1.10), and the distribution of integral points is described by the results from Section 1 (see Remark 1.21). Let us consider "generic" case, that is,

$$
2 \rho=\sum_{\alpha \in \Delta} u_{\alpha} \alpha \quad \text { and } \quad \lambda_{\iota}=\sum_{\alpha \in \Delta} m_{\alpha} \alpha=\sum_{\alpha \in \Delta} n_{\alpha} \omega_{\alpha}
$$

with all $n_{\alpha}>0$ and $\frac{u_{\alpha}}{m_{\alpha}} \neq \frac{u_{\beta}}{m_{\beta}}$ for all $\alpha \neq \beta$. Then the Satake boundary $V^{\infty}$ is a union of $2^{\operatorname{dim} \mathfrak{a}}$ orbits of $G$, and there are exactly dima open orbits $V_{\Delta \backslash\{\alpha\}}^{\infty}, \alpha \in \Delta$, but the measures

$$
T^{-\max _{\alpha}\left(u_{\alpha} / m_{\alpha}\right)} \sum_{v \in V(\mathbb{Z}):\|v\|<T} \delta_{\pi(v)}
$$

converge as $T \rightarrow \infty$ to a measure concentrated on the single open $G$-orbit $V_{\Delta \backslash\left\{\alpha_{0}\right\}}^{\infty}$ such that $\frac{u_{\alpha_{0}}}{m_{\alpha_{0}}}=\max _{\alpha} \frac{u_{\alpha}}{m_{\alpha}}$ (compare with non-generic case in Section 2.2). The number of integral points in $V$ with norm less than $T$ is of order $T^{\max _{\alpha}\left(u_{\alpha} / m_{\alpha}\right)}$ as $T \rightarrow \infty$, and 
the number of points whose projections accumulate on the open $G$-orbit $V_{\Delta \backslash\{\alpha\}}^{\infty}$ is of order $T^{u_{\alpha} / m_{\alpha}}$ as $T \rightarrow \infty$.

2.5. General affine symmetric variety. Let $G$ be a connected noncompact semisimple Lie group and $H$ a symmetric subgroup. We fix a Cartan decomposition

$$
G=K \exp \left(\mathfrak{a}^{+}\right) \mathcal{W} H .
$$

By Proposition 3.16, given an integral dominant weight $\lambda$ of $\mathfrak{a}^{+}$, there exists an $\mathbb{R}$ irreducible $H$-spherical representation $\iota: G \rightarrow \mathrm{GL}(W)$ with the highest weight $\lambda_{\iota}$ being a multiple of $\lambda$. Then $W$ contains a symmetric variety $V \simeq G / H$. The structure of the Satake boundary $V^{\infty}$ of $V$ is determined by the combinatorial data (2.2) of $2 \rho$ and $\lambda_{\iota}$. Assume that $G$ and $H$ are the groups of real points of algebraic semisimple $\mathbb{Q}$-groups $\mathbf{G}$ and $\mathbf{H}$ such that $\mathbf{G}$ is $\mathbb{Q}$-simple and $\mathbf{H}$ has no nontrivial $\mathbb{Q}$-characters, and that $\iota$ is defined over $\mathbb{Q}$. Then if $V(\mathbb{Z}) \neq \emptyset$, the distribution of integral points $V(\mathbb{Z})$ is determined by (2.2) as well. We mention two examples.

The "generic" case (i.e., all $n_{\alpha}>0$ and $\frac{u_{\alpha}}{m_{\alpha}} \neq \frac{u_{\beta}}{m_{\beta}}$ for $\alpha \neq \beta$ ) is quite similar to the discussion in Section 2.4 except that when $V$ is not a group variety, the sets $V_{I}^{\infty}$ may be unions of several $G$-orbits.

It is well known that $2 \rho$ is an integral dominant weight and all $n_{\alpha}>0$. Hence, by Proposition 3.16, there exists an $H$-spherical representation with the highest weight $\lambda_{\iota}=2 \ell \rho$ for some $\ell \in \mathbb{N}$. Moreover, if $\mathbf{G}$ is an inner form, then the corresponding representation is defined over $\mathbb{Q}$ (see Remark 3.22). We compute: $a_{\iota}=1 / \ell, b_{\iota}=\operatorname{dim} \mathfrak{a}$, $I_{\iota}=\emptyset$. Hence, the number of integral points in $V$ with norm less than $T$ is of order $T^{1 / \ell}(\log T)^{\operatorname{dim} \mathfrak{a}-1}$, and the measures

$$
\frac{1}{T^{1 / \ell}(\log T)^{\operatorname{dim} \mathfrak{a}-1}} \sum_{v \in V(\mathbb{Z}):\|v\|<T} \delta_{\pi(v)}
$$

converge as $T \rightarrow \infty$ to a measure $\mu_{\iota}$ supported on $V_{\emptyset}^{\infty}$. Note that $K$ acts transitively on $V_{\emptyset}^{\infty}$ (see Proposition 4.35), and $\mu_{\iota}=\|v\|^{-a_{\iota}} d v$ where $d v$ is a suitably normalized $K$-invariant measure on $V_{\emptyset}^{\infty}$ (cf. (7.17) ). On the other hand, for $f \in C(S(W))$ such that supp $f \cap V^{\infty} \subset V_{\Delta_{\sigma}-\{\alpha\}}^{\infty}$ for some $\alpha \in \Delta_{\sigma}$, we have

$$
T^{-1 / \ell} \sum_{v \in V(\mathbb{Z}):\|v\|<T} f(\pi(v)) \rightarrow \mu_{\Delta_{\sigma}-\{\alpha\}}(f)
$$

where $\mu_{\Delta_{\sigma}-\{\alpha\}}$ is a measure concentrated on $V_{\Delta_{\sigma}-\{\alpha\}}^{\infty}$.

\section{Affine symmetric spaces And Representations}

3.1. Affine symmetric spaces. (see [Sc, Ch. 7], [HS, Part II], $\mathrm{OS},[\mathrm{Ro}]$ )

Let $G$ be a connected noncompact semisimple Lie group with finite center and $\mathfrak{g}$ the Lie algebra of $G$. A closed subgroup $H$ of $G$, with the Lie algebra $\mathfrak{h} \subset \mathfrak{g}$, is called symmetric if $\mathfrak{h}$ is the set of fixed points of an involution $\sigma$ of $\mathfrak{g}$. Then the factor space $G / H$ is called an affine symmetric space. 
There exists a Cartan involution $\theta$ of $\mathfrak{g}$ which commutes with $\sigma$. We denote by $K$ the maximal compact subgroup of $G$ that corresponds to $\theta$ and by $\mathfrak{k}$ its Lie algebra. We have decompositions

$$
\mathfrak{g}=\mathfrak{h} \oplus \mathfrak{q} \quad \text { and } \quad \mathfrak{g}=\mathfrak{k} \oplus \mathfrak{p}
$$

into +1 and -1 eigenspaces of $\sigma$ and $\theta$ respectively.

There exists a Cartan subalgebra $\mathfrak{c}$ of $\mathfrak{g}$ stable under $\theta$ and $\sigma$ such that $\mathfrak{b}:=\mathfrak{c} \cap \mathfrak{p}$ is a maximal abelian subalgebra of $\mathfrak{p}, \mathfrak{c} \cap \mathfrak{q}$ is a maximal abelian subalgebra of $\mathfrak{q}$, and $\mathfrak{a}:=\mathfrak{c} \cap \mathfrak{p} \cap \mathfrak{q}$ is a maximal abelian subalgebra of $\mathfrak{p} \cap \mathfrak{q}$. We call $\mathfrak{b}$ a Cartan subalgebra associated to $\theta$ and $\mathfrak{a}$ a Cartan subalgebra associated to $(\theta, \sigma)$. We denote by $\Sigma_{\mathbb{C}} \subset \mathfrak{c}_{\mathbb{C}}{ }^{*}$, $\Sigma \subset \mathfrak{b}^{*}$, and $\Sigma_{\sigma} \subset \mathfrak{a}^{*}$ the root systems. One can choose a set of positive roots $\Sigma_{\mathbb{C}}^{+} \subset \Sigma_{\mathbb{C}}$ so that $\Sigma^{+}=\left.\Sigma_{\mathbb{C}}^{+}\right|_{\mathfrak{b}} \backslash\{0\}$ and $\Sigma_{\sigma}^{+}=\left.\Sigma^{+}\right|_{\mathfrak{a}} \backslash\{0\}$ are systems of positive roots in $\Sigma$ and $\Sigma_{\sigma}$

Let $\Delta_{\mathbb{C}} \subset \Sigma_{\mathbb{C}}^{+}$denote the system of simple roots. Then

$$
\Delta=\left.\Delta_{\mathbb{C}}\right|_{\mathfrak{b}} \backslash\{0\} \quad \text { and } \quad \Delta_{\sigma}=\left.\Delta\right|_{\mathfrak{a}} \backslash\{0\} .
$$

are systems of simple roots for $\Sigma$ and $\Sigma_{\sigma}$ respectively. We also set $\Delta_{0}=\{\alpha \in \Delta$ : $\left.\left.\alpha\right|_{\mathfrak{a}}=0\right\}$.

The space $\mathfrak{a}$ is a Cartan subalgebra associated to $\theta$ of the reductive Lie algebra $(\mathfrak{k} \cap \mathfrak{h}) \oplus(\mathfrak{p} \cap \mathfrak{q})$, which is the set of fixed points of the involution $\sigma \theta$. We denote by $\Sigma_{\sigma, \theta} \subset \Sigma_{\sigma}$ the corresponding root system and choose a set positive roots $\Sigma_{\sigma, \theta}^{+} \subset \Sigma_{\sigma, \theta}$ such that $\Sigma_{\sigma, \theta}^{+} \subset \Sigma_{\sigma}^{+}$.

The Weyl groups of $\Sigma_{\sigma}$ and $\Sigma_{\sigma, \theta}$ are given by

$$
\mathcal{W}_{\sigma}=N_{K}(\mathfrak{a}) / Z_{K}(\mathfrak{a}) \quad \text { and } \quad \mathcal{W}_{\sigma, \theta}=N_{K \cap H}(\mathfrak{a}) / Z_{K \cap H}(\mathfrak{a}),
$$

and one can choose a set $\mathcal{W} \subset N_{K}(\mathfrak{a}) \cap N_{K}(\mathfrak{b})$ of coset representatives of $\mathcal{W}_{\sigma} / \mathcal{W}_{\sigma, \theta}$

Denoting by $\mathfrak{a}^{+}$be the closed positive Weyl chamber for $\Sigma_{\sigma}^{+}$, we have Cartan decomposition:

$$
G=K \exp (\mathfrak{a}) H=K \exp \left(\mathfrak{a}^{+}\right) \mathcal{W} H .
$$

Note that for any $g \in G, \mathfrak{a}^{+}$-component of $g$ and the $\mathcal{W}$-component of $g$ are uniquely defined.

For a root $\alpha \in \Sigma_{\sigma} \cup\{0\}$, we denote by $\mathfrak{g}_{\alpha}$ the corresponding root space associated to a. Also for a root $\tilde{\alpha} \in \Sigma$, we denote by $\mathfrak{g}_{\tilde{\alpha}}(\mathfrak{b})$ the corresponding root space associated to $\mathfrak{b}$.

Let $\langle\cdot, \cdot\rangle$ denote the Killing form on $\mathfrak{g}$. We consider a positive definite symmetric bilinear form $B$ on $\mathfrak{g}$ :

$$
B(X, Y)=-\langle X, \theta(Y)\rangle=\operatorname{Tr}(\operatorname{ad} X \circ \operatorname{ad}(\theta(Y)) .
$$

Note that

$$
\begin{aligned}
& B\left(\mathfrak{g}_{\alpha}, \mathfrak{g}_{\beta}\right)=0 \quad \text { for all } \alpha \neq \beta \in \Sigma_{\sigma} \cup\{0\}, \\
& B^{\theta}=B^{\sigma}=B .
\end{aligned}
$$


Remark 3.5. For $\tilde{\beta} \in \Sigma$, take any $X \in \mathfrak{g}_{\tilde{\beta}}(\mathfrak{b})$ such that $B(X, X)=1$, and put $b_{\tilde{\beta}}=[X,-\theta(X)]$. Then $\theta\left(b_{\tilde{\beta}}\right)=-b_{\tilde{\beta}}$. Since $\theta\left(\mathfrak{g}_{\tilde{\beta}}\right)=\mathfrak{g}_{-\tilde{\beta}}$, we have $b_{\tilde{\beta}} \in \mathfrak{g}_{0}(\mathfrak{b})$. Hence $b_{\tilde{\beta}} \in \mathfrak{b}$. Moreover for all $b \in \mathfrak{b}$,

$$
\left\langle b, b_{\tilde{\beta}}\right\rangle=\langle b,[X,-\theta(X)]\rangle=\langle[b, X],-\theta(X)\rangle=\tilde{\beta}(b)\langle X,-\theta(X)\rangle=\tilde{\beta}(b) .
$$

Since the Killing form restricted to $\mathfrak{b}$ is nondegenerate, $\mathfrak{b}_{\tilde{\beta}}$ is the unique element, say $b_{*}$, of $\mathfrak{b}$ such that $\left\langle b, b_{*}\right\rangle=\tilde{\beta}(b)$ for all $b \in \mathfrak{b}$.

For each $\alpha \in \Sigma_{\sigma}$, the root space $\mathfrak{g}_{\alpha}$ is invariant under the involution $\sigma \theta$, and it decomposes into $( \pm 1)$-eigenspaces of $\sigma \theta$ :

$$
\mathfrak{g}_{\alpha}=\mathfrak{g}_{\alpha}^{+} \oplus \mathfrak{g}_{\alpha}^{-} ;
$$

we define

$$
l_{\alpha}^{ \pm}=\operatorname{dim} \mathfrak{g}_{\alpha}^{ \pm}, \quad \text { and } \quad l_{\alpha}=l_{\alpha}^{+}+l_{\alpha}^{-}
$$

We have

$$
2 \rho=\sum_{\alpha \in \Sigma_{\sigma}^{+}} l_{\alpha} \alpha .
$$

A Haar measure on $G / H$ is given by the formula

$$
\int_{G / H} f d \mu=\int_{K} \sum_{w \in \mathcal{W}} \int_{\mathfrak{a}^{+}} f(k \exp (a) w H) \xi(a) d a d k, \quad f \in C_{c}(G / H),
$$

where $d a$ and $d k$ denote Haar measures on $\mathfrak{a}$ and $K$, and

$$
\xi(a)=\prod_{\alpha \in \Sigma_{\sigma}^{+}}(\sinh \alpha(a))^{l_{\alpha}^{+}}(\cosh \alpha(a))^{l_{\alpha}^{-}} .
$$

To match (3.7) with the integral formula given in [Sc, Ch. 7], we note that the function $|\xi|$ is invariant under the Weyl group.

Remark 3.9. For $\alpha \in \Sigma_{\sigma}$, let $X \in \mathfrak{g}_{\alpha}^{+} \cup \mathfrak{g}_{\alpha}^{-}$such that $B(X, X)=1$ and put $a_{\alpha}:=$ $[X,-\theta(X)] \in \mathfrak{g}_{0}$. Then $\sigma\left(a_{\alpha}\right)=-a_{\alpha}$ and $\theta\left(a_{\alpha}\right)=-a_{\alpha}$. Therefore $a_{\alpha} \in \mathfrak{a}$ and by (3.6), we have $\left\langle a, a_{\alpha}\right\rangle=\alpha(a)$ for all $a \in \mathfrak{a}$. Hence $a_{\alpha}$ is the unique element of $\mathfrak{a}$ satisfying the last equation.

3.2. Representations. (see [GJT, Ch. IV], $[\mathrm{Sa},[\mathrm{CP})$ Let $\iota: G \rightarrow \mathrm{GL}(W)$ be an irreducible over $\mathbb{R}$ representation of $G$ on a real vector space $W$. We denote by $\mathfrak{g}_{\mathbb{C}}, \mathfrak{c}_{\mathbb{C}}$, $\mathfrak{h}_{\mathbb{C}}$ the complexifications of $\mathfrak{g}, \mathfrak{c}, \mathfrak{h}$. Note that $\sigma$ extends to an involution of $\mathfrak{g}_{\mathbb{C}}$, and $\mathfrak{h}_{\mathbb{C}}$ is the subalgebra of the fixed points of $\sigma$ in $\mathfrak{g}_{\mathbb{C}}$.

Let $W_{0}$ be a complex $\mathfrak{g}$-irreducible subspace of $\mathbb{C} \otimes W$. Then either $\mathbb{C} \otimes W=W_{0}$ or $\mathbb{C} \otimes W=W_{0} \oplus \bar{W}_{0}$, where bar denotes the standard complex conjugation on $\mathbb{C} \otimes W$. Note that if $\mathbb{C} \otimes W$ is not complex irreducible, then the map

$$
W_{0} \ni v \mapsto(v+\bar{v}) \in W
$$

is a $\mathfrak{g}$-equivariant isomorphism over $\mathbb{R}$; and hence in this case $W$ can be treated as vector space over $\mathbb{C}$ with $\mathbb{C}$-linear action of $\mathfrak{g}$. By abuse of notation, the representation of $\mathfrak{g}$ on $W_{0}$ over $\mathbb{C}$ will also be denoted by $\iota$. 
We denote by $\Lambda_{\iota} \in \mathfrak{c}_{\mathbb{C}}^{*}$ the highest weight of $\iota$ with respect to the ordering defined by $\Delta_{\mathbb{C}}$. Then all other weights of $\mathfrak{c}_{\mathbb{C}}$ with respect to $\iota$ are of the form

$$
\lambda=\Lambda_{\iota}-\sum_{\alpha \in \Delta_{\mathbb{C}}} n_{\alpha}(\lambda) \alpha
$$

for some non-negative integers $n_{\alpha}(\lambda)$.

The action of $\mathfrak{a}$ on $W$ is diagonalizable (over $\mathbb{R}$ ) and

$$
W=\oplus_{\lambda \in \Phi_{\iota}} W^{\lambda},
$$

where $\Phi_{\iota} \subset \mathfrak{a}^{*}$ is the set of weights and

$$
W^{\lambda}=\{w \in W: a \cdot w=\lambda(a) w, \forall a \in \mathfrak{a}\}
$$

denotes the weight space with weight $\lambda$. Given $w \in W$, we have a decomposition

$$
w=\sum_{\lambda \in \Phi_{\iota}} w^{\lambda}, \quad w^{\lambda} \in W^{\lambda}
$$

The weight $\lambda_{\iota}:=\left.\Lambda_{\iota}\right|_{\mathfrak{a}}$ is the maximal element of $\Phi_{\iota}$ with respect to the ordering defined by $\Delta_{\sigma}$. All the other weights $\lambda \in \Phi_{\iota}$ are of the form

$$
\lambda=\lambda_{\iota}-\sum_{\alpha \in \Delta_{\sigma}} n_{\alpha}(\lambda) \alpha
$$

for some non-negative integers $n_{\alpha}(\lambda)$. Let

$$
\operatorname{supp} \lambda=\left\{\alpha \in \Delta_{\sigma}: n_{\alpha}(\lambda)>0\right\} .
$$

For a subset $I$ of $\Delta_{\sigma}$ and a vector $w \in W$, we set

$$
w^{I}=\sum_{\lambda: \operatorname{supp} \lambda \subset I} w^{\lambda} \text { and } W^{I}=\sum_{\lambda: \operatorname{supp} \lambda \subset I} W^{\lambda} .
$$

Recalling Definition 1.14, a subset of $\mathfrak{a}^{*}$ is called connected if it is not a union of nonempty subsets orthogonal with respect to the form $B$; that is, if its Dynkin diagram is connected. We say that $I \subset \Delta_{\sigma}$ is $\lambda_{\iota}$-connected, if $I \cup\left\{\lambda_{\iota}\right\}$ is a connected subset of $\Delta_{\sigma}$.

Proposition 3.13. For any $\lambda \in \Phi_{\iota}, \operatorname{supp}(\lambda) \cup\left\{\lambda_{\iota}\right\}$ is connected, and for every $\lambda_{\iota}$ connected $I \subset \Delta_{\sigma}$ there exists $\lambda \in \Phi_{\iota}$ such that $\operatorname{supp}(\lambda)=I$.

Proof. The similar statement for the set of weight of $\mathfrak{b}$ was shown in [Sa, Sec. 2], and the proof applies to our situation with minor changes. The key fact is that there exists an involution $\alpha \mapsto \alpha^{\prime}$ of the set $\Delta-\Delta_{0}$ such that

$$
-\alpha^{\sigma}=\alpha^{\prime}+\sum_{\beta \in \Delta_{0}} n_{\alpha, \beta} \beta, \quad \alpha \in \Delta \backslash \Delta_{0},
$$

for some $n_{\alpha, \beta} \in \mathbb{Z}_{\geq 0}$ (see [Sc, Lemma 7.2.3]). Using that the proposition holds for the weights of $\mathfrak{b}$, one can complete the proof as in [Sa].

Remark 3.14. We set $\mathbb{K}=\mathbb{R}$ when $W \otimes \mathbb{C}=W_{0}$, and $\mathbb{K}=\mathbb{C}$ when $W \otimes \mathbb{C}=W_{0} \oplus \bar{W}_{0}$. Then $W$ can be treated as a $\mathbb{K}$-vector space with $\mathbb{K}$-linear action of $\mathfrak{g}$. 
$H$-spherical representations. Let $W^{H}$ denote the space of fixed points of $H$ on $W$. If $W^{H} \neq 0$, then the representation $\iota$ is called $H$-spherical.

Lemma 3.15 ([CP, Lemma 1.5]). If $\iota$ is $H$-spherical then the $\mathbb{K}-\operatorname{dim}\left(W^{\mathfrak{h}}\right)=1$; and $\Lambda_{\iota}^{\sigma}=-\Lambda_{\iota}$.

Using the form $B$ defined in (3.3), we introduce a scalar product on $\mathfrak{c}^{*}, \mathfrak{b}^{*}, \mathfrak{a}^{*}$. An element $\lambda \in \mathfrak{c}^{*}$ is called integral if $\frac{2\langle\lambda, \alpha\rangle}{\langle\alpha, \alpha\rangle} \in \mathbb{Z}$ for all $\alpha \in \Delta_{\mathbb{C}}$, and it is called dominant if $\langle\lambda, \alpha\rangle \geq 0$ for all $\alpha \in \Delta_{\mathbb{C}}$. For $\beta \in \Delta_{\mathbb{C}}$, we define the fundamental weights $\omega_{\beta}$ by

$$
\frac{2\left\langle\omega_{\beta}, \alpha\right\rangle}{\langle\alpha, \alpha\rangle}=\delta_{\alpha \beta}, \quad \forall \alpha \in \Delta_{\mathbb{C}}
$$

where $\delta_{\alpha \beta}$ denotes the Kronecker symbol. Similarly, we define these notions for $\mathfrak{b}^{*}$ and $\mathfrak{a}^{*}$. It is well-known that the the highest weight $\Lambda_{\iota}$ is integral and dominant, and conversely, every integral dominant weight is the highest weight of an irreducible representation of $\mathfrak{g}_{\mathbb{C}}$. We prove an analogous result for real spherical representations:

Proposition 3.16 (cf. GJT, Proposition 4.15]). The highest weight $\lambda_{\iota}$ is integral and dominant. There exists $\ell \in \mathbb{N}$ such that for every integral dominant $\lambda \in \mathfrak{a}^{*}, \ell \lambda$ is a highest weight of a real absolutely irreducible $H$-spherical representation of $\mathfrak{g}$.

Proof. The fact that $\lambda_{\iota}$ is integral and dominant follows from the representation theory of $\mathfrak{s l}(2, \mathbb{R})$ (see GJT, Lemma 4.12]).

For $\alpha \in \Delta_{\mathbb{C}}\left(\right.$ or $\alpha \in \Delta_{\sigma}$ ), we take $h_{\alpha} \in \mathfrak{c}$ and $h_{\alpha}^{*} \in \mathfrak{c}^{*}\left(\right.$ or $h_{\alpha} \in \mathfrak{a}$ and $h_{\alpha}^{*} \in \mathfrak{a}^{*}$ ) such that

$$
\left\langle h_{\beta}, h_{\alpha}\right\rangle=\beta\left(h_{\alpha}\right)=\langle\beta, \alpha\rangle \quad \text { and } \quad\left\langle h_{\alpha}^{*}, \beta\right\rangle=h_{\alpha}^{*}\left(h_{\beta}\right)=\delta_{\alpha \beta}
$$

for all $\alpha, \beta \in \Delta_{\mathbb{C}}$ (or $\alpha, \beta \in \Delta_{\sigma}$ ). For $\lambda \in \mathfrak{c}^{*}$, we denote by $\bar{\lambda} \in \mathfrak{a}^{*}$ its restriction to $\mathfrak{a}$, and for $x \in \mathfrak{c}$, we denote by $\bar{x} \in \mathfrak{a}$ its orthogonal projection to $\mathfrak{a}$. It follows from (3.1) and (3.4) that for $\alpha \in \Delta_{\mathbb{C}}$ such that $\bar{\alpha} \neq 0$ and $\beta \in \Delta_{\sigma}$,

$$
\bar{h}_{\alpha}=h_{\bar{\alpha}} \quad \text { and } \quad h_{\beta}^{*}=\sum_{\alpha \in \Delta_{\mathbb{C}}: \bar{\alpha}=\beta} \bar{h}_{\alpha}^{*} .
$$

Suppose that $\lambda=\sum_{\beta \in \Delta_{\sigma}} n_{\beta} \omega_{\beta}$ for $n_{\beta} \in \mathbb{Z}_{\geq 0}$. Since $\omega_{\beta}=\frac{1}{2}\langle\beta, \beta\rangle h_{\beta}^{*}$, we have

$$
\lambda=\sum_{\alpha \in \Delta_{\mathbb{C}}: \bar{\alpha} \neq 0} n_{\bar{\alpha}} \frac{\langle\bar{\alpha}, \bar{\alpha}\rangle}{\langle\alpha, \alpha\rangle} \cdot \bar{\omega}_{\alpha} .
$$

It is well known that $\left\langle\alpha_{1}, \alpha_{2}\right\rangle \in \mathbb{Q}$ for $\alpha_{1}, \alpha_{2} \in \Sigma_{\mathbb{C}}$. Hence, using that

$$
\bar{\alpha}=\frac{1}{4}\left(\alpha-\alpha^{\theta}-\alpha^{\sigma}+\alpha^{\sigma \theta}\right),
$$

we deduce that the coefficients in (3.17) are rational numbers. We take $\ell \in \mathbb{N}$ such that

$$
\ell \lambda=\sum_{\alpha \in \Delta_{\mathbb{C}}: \bar{\alpha} \neq 0} m_{\alpha} \bar{\omega}_{\alpha}
$$


for $m_{\alpha} \in 2 \mathbb{Z}_{\geq 0}$ and consider an irreducible complex representation $\iota: \mathfrak{g}_{\mathbb{C}} \rightarrow \operatorname{GL}\left(W_{0}\right)$ with the highest weight

$$
\Lambda=\sum_{\alpha \in \Delta_{\mathbb{C}}: \bar{\alpha} \neq 0} m_{\alpha} \omega_{\alpha}
$$

Let $\Delta_{\mathbb{C}}^{\theta}=\left\{\alpha \in \Delta_{\mathbb{C}}:\left.\alpha\right|_{\mathfrak{b}}=0\right\}$. It was shown in [Sa] that there exists an involution $\alpha \mapsto \tilde{\theta}(\alpha)$ of the set $\Delta_{\mathbb{C}} \backslash \Delta_{\mathbb{C}}^{\theta}$ such that

$$
-\alpha^{\theta}=\tilde{\theta}(\alpha)+\sum_{\beta \in \Delta_{\mathbb{C}}^{\theta}} u_{\alpha, \beta} \beta, \quad \alpha \in \Delta_{\mathbb{C}} \backslash \Delta_{\mathbb{C}}^{\theta},
$$

for some $u_{\alpha, \beta} \in \mathbb{Z}_{\geq 0}$. Moreover, according to [On, $\S 9$ ], the involution $\tilde{\theta}$ is induced by an automorphism of the Dynkin diagram of $\Delta_{\mathbb{C}}$. In particular, $\langle\tilde{\theta}(\alpha), \tilde{\theta}(\alpha)\rangle=\langle\alpha, \alpha\rangle$ for all $\alpha \in \Delta_{\mathbb{C}} \backslash \Delta_{\mathbb{C}}^{\theta}$. Also, it is clear that $\left.\alpha\right|_{\mathfrak{a}}=\left.\tilde{\theta}(\alpha)\right|_{\mathfrak{a}}$. This shows that $m_{\alpha}=m_{\tilde{\theta}(\alpha)}$ and by [On, §8], the restriction of $\iota$ to $\mathfrak{g}$ leaves the a real form $W$ of $W_{0}$ invariant.

It remain to check that the representation $\iota$ is spherical. Recall that $\mathfrak{d}:=\mathfrak{c} \cap \mathfrak{q}=\{x \in$ $\mathfrak{c}: \sigma(x)=-x\}$ is a maximal abelian subalgebra of $\mathfrak{q}$. Let $\Delta_{\mathbb{C}}^{\sigma}=\left\{\alpha \in \Delta_{\mathbb{C}}:\left.\alpha\right|_{\mathfrak{d}}=0\right\}$. It was shown in $\left[\mathrm{CP}\right.$ that there exists an involution $\alpha \mapsto \tilde{\sigma}(\alpha)$ of the set $\Delta_{\mathbb{C}} \backslash \Delta_{\mathbb{C}}^{\sigma}$ such that

$$
-\alpha^{\sigma}=\tilde{\sigma}(\alpha)+\sum_{\beta \in \Delta_{\mathbb{C}}^{\sigma}} v_{\alpha, \beta} \beta, \quad \alpha \in \Delta_{\mathbb{C}} \backslash \Delta_{\mathbb{C}}^{\sigma}
$$

for some $v_{\alpha, \beta} \in \mathbb{Z}_{\geq 0}$. Since $m_{\alpha}$ 's are even, according to [CP], the representation $\iota$ is spherical provided that $m_{\alpha}=m_{\tilde{\sigma}(\alpha)}$ for $\alpha \in \Delta_{\mathbb{C}} \backslash \Delta_{\mathbb{C}}^{\sigma}$. Hence, it suffices to show that the involution $\tilde{\sigma}$ is induced by an automorphism of the Dynkin diagram of $\Delta_{\mathbb{C}}$. Without loss of generality, we may assume that $\Delta_{\mathbb{C}}^{\sigma} \neq \emptyset$. Then one can check that $\mathfrak{c} \cap \mathfrak{h}$ is a Cartan subalgebra of $\left[\mathfrak{z}_{\mathfrak{h}}(\mathfrak{d}), \mathfrak{z}_{\mathfrak{h}}(\mathfrak{d})\right]$ with the system of simple roots $\Delta_{\mathbb{C}}^{\sigma}$. The corresponding Weyl group $\mathcal{W}_{\mathbb{C}}^{\sigma}$ is generated by reflections

$$
w_{\beta}(\alpha)=\alpha-2 \frac{\langle\alpha, \beta\rangle}{\langle\beta, \beta\rangle} \beta, \quad \beta \in \Delta_{\mathbb{C}}^{\sigma}
$$

This implies that for every $w \in \mathcal{W}_{\mathbb{C}}^{\sigma}$,

$$
\begin{aligned}
\left(\Sigma_{\mathbb{C}}^{+} \backslash\left\langle\Delta_{\mathbb{C}}^{\sigma}\right\rangle\right)^{w} & \subset \Sigma_{\mathbb{C}}^{+} \backslash\left\langle\Delta_{\mathbb{C}}^{\sigma}\right\rangle, \\
\alpha^{w} & \in \alpha+\left\langle\Delta_{\mathbb{C}}^{\sigma}\right\rangle, \quad \alpha \in \Delta_{\mathbb{C}} .
\end{aligned}
$$

Take $w_{0} \in \mathcal{W}_{\mathbb{C}}^{\sigma}$ such that $\left(\Delta_{\mathbb{C}}^{\sigma}\right)^{w_{0}}=-\Delta_{\mathbb{C}}^{\sigma}$. It follows from (3.19) and (3.20) that the map $\alpha \mapsto-\alpha^{\sigma w_{0}}$ preserves $\Sigma_{\mathbb{C}}^{+}$, and hence, it induces an automorphism of the Dynkin diagram of $\Delta_{\mathbb{C}}$. On the other hand, it follows from (3.19) and (3.21) that for $\alpha \in \Delta_{\mathbb{C}} \backslash \Delta_{\mathbb{C}}^{\sigma}$,

$$
-\alpha^{\sigma w_{0}} \in \tilde{\sigma}(\alpha)+\left\langle\Delta_{\mathbb{C}}^{\sigma}\right\rangle .
$$

This implies that $\tilde{\sigma}(\alpha)=-\alpha^{\sigma w_{0}}, \alpha \in \Delta_{\mathbb{C}} \backslash \Delta_{\mathbb{C}}^{\sigma}$, and finishes the proof.

Remark 3.22. Suppose that $G=\mathbf{G}(\mathbb{R})^{o}$ for a semisimple algebraic $\mathbb{Q}$-group $\mathbf{G}$. Choosing the Cartan subalgebra $\mathfrak{c}$ to be defined over $\mathbb{Q}$, we have the $\star$-action of the 
Galois group $\operatorname{Gal}(\overline{\mathbb{Q}} / \mathbb{Q})$ on the set of simple roots $\Delta_{\mathbb{C}}$. By $[\mathbb{T}$, Theorem 3.2], the representation constructed in Proposition 3.16 is defined over $\mathbb{Q}$ provided that the highest weight $\Lambda$ is in the root lattice, and the coefficients in (3.18) are invariant under the $\star$-action. In particular, if $\mathbf{G}$ is an inner form, then the $\star$-action is trivial, and $\ell \lambda$ is realized as a highest weight of a representation defined over $\mathbb{Q}$ for some $\ell$.

\section{Structure of Satake Compactification}

Let $G$ be a connected noncompact semisimple Lie group with a finite center and $\iota$ : $G \rightarrow \mathrm{GL}_{\mathbb{R}}(W)$ an irreducible almost faithful representation of $G$ on a finite dimensional real vector space $W$. Let $\sigma$ be an involution of $G$ and $H$ the symmetric subgroup of $G$ with respect to $\sigma$. We assume that $H$ fixes a nonzero $v_{0} \in W$.

We start with some basic observations: let

$$
\mathfrak{n}=\sum_{\alpha \in \Sigma_{\sigma}^{+}} \mathfrak{g}_{\alpha}
$$

Lemma 4.1. We have $W^{\lambda_{\iota}}=\{v \in W: \mathfrak{n} v=0\}$.

Proof. Let $\alpha \in \Sigma_{\sigma}^{+}$. Then $\mathfrak{g}_{\alpha} W^{\lambda_{\iota}} \subset W^{\lambda_{\iota}+\alpha}$. Since $\lambda_{\iota}$ is the highest weight in $\Phi_{\iota}$, we conclude that $W^{\lambda_{\iota}+\alpha}=0$. This shows that $\mathfrak{n} W^{\lambda_{\iota}}=0$.

Now let $v \in W$ such that $\mathfrak{n} v=0$. Suppose $v \notin W^{\lambda_{\iota}}$. Then there exists $y \in W^{\prime}:=$ $\sum_{\lambda<\lambda_{\iota}} W^{\lambda}$ such that $\mathfrak{n} y=0$. Let $\mathfrak{n}^{-}=\sum_{\alpha \in \Sigma_{\sigma}^{+}} \mathfrak{g}_{-\alpha}$. Then $\mathfrak{g}=\mathfrak{n}^{-} \oplus \mathfrak{g}_{0} \oplus \mathfrak{n}$. Note that

$$
U_{0}(\mathfrak{n}) y=0 ; \quad U_{0}\left(\mathfrak{g}_{0}\right) W^{\prime} \subset W^{\prime} ; \quad U_{0}\left(\mathfrak{n}^{-}\right) W^{\prime} \subset W^{\prime},
$$

where $U_{0}(\mathfrak{n})$ denotes the linear span of (non-constant) monomials formed from a basis of $\mathfrak{n}$, and the others are defined similarly. By Poincare-Birkhoff-Witt's theorem, it follows that

$$
U(\mathfrak{g}) y \subset W^{\prime},
$$

where $U(\mathfrak{g})$ is the universal enveloping algebra of $\mathfrak{g}$. This is a contradiction, because $U(\mathfrak{g}) y=W$ by the irreducibility of the $\mathfrak{g}$-action on $W$.

Lemma 4.2. For every $\lambda_{\iota}$-connected $I \subset \Delta_{\sigma}$ and every $w \in \mathcal{W}$, there exists $\lambda \in \Phi_{\iota}$ such that $\operatorname{supp} \lambda=I$ and $\left(w v_{0}\right)^{\lambda} \neq 0$.

Proof. First, we consider the case of $I=\emptyset$; that is, we show that $\left(w v_{0}\right)^{\lambda_{\iota}} \neq 0$. We denote by $\sigma_{w}=\operatorname{Ad}(w) \circ \sigma \circ \operatorname{Ad}\left(w^{-1}\right)$ the involution of $\mathfrak{g}$ corresponding to the symmetric subgroup $w H w^{-1}$. Take a maximal $\lambda \in \Phi_{\iota}$ such that $\left(w v_{0}\right)^{\lambda} \neq 0$ and suppose that $\lambda \neq \lambda_{\iota}$. Then by Lemma 4.1 there exist $\alpha \in \Sigma_{\sigma}^{+}$and $X \in \mathfrak{g}_{\alpha}$ such that $X\left(w v_{0}\right)^{\lambda} \neq 0$. Since $X+\sigma_{w}(X)$ belongs to the Lie algebra of $w H w^{-1}$,

$$
\left(X+\sigma_{w}(X)\right)\left(w v_{0}\right)=0 .
$$

Therefore there exists $\mu \in \Phi_{\iota}$ such that $\left(w v_{0}\right)^{\mu} \neq 0$ and $\lambda+\alpha=\mu+\alpha^{\sigma_{w}}$. Since $\sigma_{w}(a)=-a$ for all $a \in \mathfrak{a}$, we have $\alpha^{\sigma_{w}}=-\alpha$. Therefore $\mu=\lambda+2 \alpha>\lambda$, which contradicts the choice of $\lambda$. 
Now we prove the general case. Given a $\lambda_{\iota}$-connected $I \subset \Delta_{\sigma}$, there exists $w_{0} \in \mathcal{W}_{\sigma}$ such that the weight $\lambda_{\iota} \circ \operatorname{Ad}\left(w_{0}\right)$ has support equal to $I$ (GJT, Lemma B.8]). Then by the above case

$$
w_{0}\left(w v_{0}\right)^{\lambda_{\iota} \circ \mathrm{Ad} w_{0}}=\left(w_{0} w v_{0}\right)^{\lambda_{\iota}} \neq 0 .
$$

This proves the lemma.

\subsection{Symmetric subgroup as a stabilizer.}

Proposition 4.3. The map $G / H \rightarrow V$ given by $g H \mapsto g v_{0}$, for all $g \in G$, is proper. In particular, the orbit $G v_{0}$ is closed.

Proof. Take any $w \in \mathcal{W}$. By Lemma $4.2\left(w v_{0}\right)^{\lambda_{\iota}} \neq 0$. Since the representation is almost faithful,

$$
\lambda_{\iota}=\sum_{\alpha \in \Delta_{\sigma}} m_{\alpha} \alpha
$$

where $m_{\alpha}>0$ for all $\alpha$. Therefore the map

$$
a \mapsto \exp (a)\left(w v_{0}\right)=\sum_{\lambda \in \Phi_{\iota}} e^{\lambda(a)}\left(w v_{0}\right)^{\lambda}
$$

from $\mathfrak{a}^{+}$to $V$ is proper. Now, since $G=K \exp \left(\mathfrak{a}^{+}\right) \mathcal{W} H$, the map $g \mapsto g v_{0}$ is proper.

Proposition 4.4. $H$ is a subgroup of finite index in $\operatorname{Stab}_{G}\left(v_{0}\right)$.

Proof. Let $L=\operatorname{Stab}_{G}\left(v_{0}\right)$. Then $H \subset L$, and by Proposition $4.3 L / H$ is compact. Therefore, since $H$ is reductive and $L$ is a real almost algebraic subgroup of $G$, we conclude that $L$ unipotent radical of $L$ is trivial. Hence $L$ is reductive. Let $\mathfrak{l}$ denote the Lie subalgebra of $\mathfrak{g}$ associated to $L$, and $\mathfrak{l}^{\perp}=\{X \in \mathfrak{g}:\langle X, \mathfrak{l}\rangle=0\}$. Since $\mathfrak{l}$ is reductive, the Killing form of $\mathfrak{g}$ restricted to $\mathfrak{l}$ is nondegenerate. Therefore we get

$$
\left[\mathfrak{l}, \mathfrak{l}^{\perp}\right] \subset \mathfrak{l}^{\perp} \text { and } \mathfrak{g}=\mathfrak{l} \oplus \mathfrak{l}^{\perp} .
$$

Since $H$ is a symmetric subgroup and $H \subset L$, we note that

$$
\mathfrak{h} \cap \mathfrak{l}^{\perp}=\{0\},\left[\mathfrak{h}^{\perp}, \mathfrak{h}^{\perp}\right] \subset \mathfrak{h},[\mathfrak{h}, \mathfrak{h}] \subset \mathfrak{h} \text {, and }\left[\mathfrak{h}, \mathfrak{h}^{\perp}\right] \subset \mathfrak{h}^{\perp}
$$

Put, $\mathfrak{m}=\mathfrak{h}^{\perp} \cap \mathfrak{l}$. Then

$$
\begin{aligned}
{\left[\mathfrak{l}^{\perp}, \mathfrak{m}\right] } & \subset\left[\mathfrak{l}^{\perp}, \mathfrak{h}^{\perp}\right] \cap\left[\mathfrak{l}^{\perp}, \mathfrak{l}\right] \subset\left[\mathfrak{h}^{\perp}, \mathfrak{h}^{\perp}\right] \cap\left[\mathfrak{l}^{\perp}, \mathfrak{l}\right] \subset \mathfrak{h} \cap \mathfrak{l}^{\perp}=\{0\}, \\
{[\mathfrak{h}, \mathfrak{m}] } & \subset\left[\mathfrak{h}, \mathfrak{h}^{\perp}\right] \cap[\mathfrak{h}, \mathfrak{l}] \subset \mathfrak{h}^{\perp} \cap \mathfrak{l}=\mathfrak{m}, \\
{[\mathfrak{m}, \mathfrak{m}] } & \subset\left[\mathfrak{h}^{\perp}, \mathfrak{h}^{\perp}\right] \subset \mathfrak{h} .
\end{aligned}
$$

We put $\mathfrak{m}_{1}=[\mathfrak{m}, \mathfrak{m}] \oplus \mathfrak{m}$. Then $\mathfrak{m}_{1}$ is stable under $\operatorname{ad}(\mathfrak{h}), \operatorname{ad}\left(\mathfrak{l}^{\perp}\right)$, and $\operatorname{ad}(\mathfrak{m})$. Since $\mathfrak{h}^{\perp}=\mathfrak{m}+\mathfrak{l}^{\perp}$, we conclude that $\mathfrak{m}_{1}$ is an ideal in $G$. Since $\mathfrak{m}_{1} \subset \mathfrak{l}$, we have that $\mathfrak{m}_{1} \cdot v_{0}=0$. Hence

$$
\mathfrak{m}_{1} \cdot X v_{0} \subset X \cdot \mathfrak{m}_{1} \cdot v_{0}+\left[\mathfrak{m}_{1}, X\right] \cdot v_{0}=\{0\}, \quad \forall X \in \mathfrak{g} .
$$

Therefore, since $\mathfrak{g}$ acts irreducibly on $W, \mathfrak{m}_{1}$ acts trivially on $W$. Since $G$ acts almost faithfully on $W$, we get $\mathfrak{m}_{1}=\{0\}$, and hence $\mathfrak{l}=\mathfrak{h}$. Now the conclusion of the proposition follows because $L / H$ is compact. 
Using Proposition 4.3 and the proof of Proposition 4.4 it is straightforward to deduce the following.

Corollary 4.7. Suppose that $G$ acts linearly and almost faithfully on a finite dimensional vector space $E$ over $\mathbb{K}=\mathbb{R}$ or $\mathbb{C}$. Suppose that there exists $0 \neq w_{0} \in E$ such that $H w_{0}=w_{0}$ and $\mathbb{K}-\operatorname{span}\left(G w_{0}\right)=E$. Then the map $g H \mapsto g v$ from $G / H$ to $E$ is proper. Moreover $H$ is a subgroup of finite index in $\operatorname{Stab}_{G}\left(w_{0}\right)$.

Let $S(W)$ denote the unit sphere in $W$, and $\pi: W \backslash\{0\} \rightarrow S(W)$ denote the radial projection.

Proposition 4.8. The map $\pi: V \rightarrow \pi(V)$ is a homeomorphism.

Proof. To verify that the map $\pi$ is bijective, we suppose that $g_{1} v_{0}=\lambda g_{2} v_{0}$ for some $g_{1}, g_{2} \in G$ and $\lambda \neq \pm 1$. Then it follows that for some $g \in G$ and $\lambda \in(-1,1), g v_{0}=\lambda v_{0}$. Therefore $g^{n} v_{0} \rightarrow 0$ as $n \rightarrow \infty$, which contradicts the conclusion of Proposition 4.3 that $G v_{0}$ is closed.

It is clear that the map is continuous and $G$-equivariant. Since the orbits of $G$ in the projective space of $W$ are locally closed, it follows that $\pi\left(G \cdot v_{0}\right)$ is locally compact. Hence, the map $\pi$ is a homeomorphism.

4.2. Satake Boundary. We define the Satake boundary $V^{\infty}$ of $V$ to be the set of the limit points of the sequences $\pi\left(v_{n}\right), v_{n} \in V, v_{n} \rightarrow \infty$. Note that identifying $G / H$ with $\pi(V)$, the space $\pi(V) \cup V^{\infty}$ gives a compactification of $G / H$ similar to the Satake compactification of the Riemannian symmetric space of $G$ constructed in [Sa].

We use notations from Section 3, For $J \subset \Delta_{\sigma}$, let $\mathfrak{a}_{J}=\operatorname{ker}(J), \mathfrak{a}^{J}$ its orthogonal complement, and

$$
\mathfrak{a}^{J,+}=\left\{a \in \mathfrak{a}^{J}: \alpha(a) \geq 0 \text { for all } \alpha \in J\right\} .
$$

The set $J$ is the system of simple roots on $\mathfrak{a}^{J}$, and its Weyl group $\mathcal{W}_{J}$ can be identified with the subgroup of $\mathcal{W}_{\sigma}$ that acts trivially on $\mathfrak{a}_{J}$. We choose a set $\mathcal{W}^{J}$ of representatives of the double cosets $\mathcal{W}_{J} \backslash \mathcal{W}_{\sigma} / \mathcal{W}_{\sigma, \theta}$. In particular, $\mathcal{W}=\mathcal{W}^{\emptyset}$.

For $J \subset \Delta_{\sigma}$ and $w \in \mathcal{W}$, we set

$$
V_{J, w}^{\infty}=\left\{\lim \pi\left(k \exp (a) w v_{0}\right): \begin{array}{l}
k \in K, a \in \mathfrak{a}^{+}, \\
\alpha(a) \rightarrow \infty \text { for } \alpha \in \Delta_{\sigma} \backslash J, \\
\alpha(a) \text { is bounded for } \alpha \in J .
\end{array}\right\} .
$$

The main result of this section is the following theorem, which gives an explicit combinatorial description of the decomposition of $V^{\infty}$ into $G$-orbits.

Define

$$
\mathcal{O}_{J, w}=\bigcup_{w_{1} \in \mathcal{W}_{J}} V_{J, w_{1} w}^{\infty}
$$

Theorem 4.10. The decomposition of $V^{\infty}$ into $G$-orbits is given by

$$
V^{\infty}=\bigcup_{I, w} \mathcal{O}_{I, w}
$$

where the union is taken over all $\lambda_{\iota}$-connected subsets $I \subsetneq \Delta_{\sigma}$ and $w \in \mathcal{W}^{I}$. 
Moreover,

$$
\mathcal{O}_{I, w}=\pi\left(G\left(w v_{0}\right)^{I}\right)
$$

and

$$
\mathcal{O}_{I_{1}, w_{1}} \cap \mathcal{O}_{I_{2}, w_{2}}=\emptyset \quad \text { for } I_{1} \neq I_{2} \text {. }
$$

We shall prove this theorem through a series of auxiliary results.

Proposition 4.13. Let $J \subset \Delta_{\sigma}, w \in \mathcal{W}$, and $I \subset J$ be the largest $\lambda_{\iota}$-connected subset. Then

$$
V_{J, w}^{\infty}=\pi\left(K \exp \left(\mathfrak{a}^{I,+}\right)\left(w v_{0}\right)^{I}\right)=\pi\left(K \exp \left(\mathfrak{a}^{J,+}\right)\left(w v_{0}\right)^{J}\right)=V_{I, w}^{\infty} .
$$

Proof. Recall that $V_{J, w}^{\infty}$ is the set of limit points of the sequences $\pi\left(k \exp \left(a_{n}\right) w v_{0}\right)$ where $k \in K$ and $\left\{a_{n}\right\} \subset \mathfrak{a}^{+}$such that $\alpha\left(a_{n}\right) \rightarrow \infty$ for $\alpha \in \Delta_{\sigma} \backslash J$ and $\alpha\left(a_{n}\right)$ is bounded for $\alpha \in J$. Passing to a subsequence, we may assume that there exists $a \in \mathfrak{a}^{I,+}$ such that $\alpha\left(a_{n}\right) \rightarrow \alpha(a)$ for every $\alpha \in I$. Then for $\lambda=\lambda_{\iota}-\sum_{\alpha \in \Delta_{\sigma}} n_{\alpha}(\lambda) \alpha \in \Phi_{\iota}$,

$$
\sum_{\alpha \in \Delta_{\sigma}} n_{\alpha}(\lambda) \alpha\left(a_{n}\right) \rightarrow \begin{cases}\sum_{\alpha \in \Delta_{\sigma}} n_{\alpha}(\lambda) \alpha(a) & \text { if } \operatorname{supp}(\lambda) \subseteq I, \\ +\infty & \text { if } \operatorname{supp}(\lambda) \nsubseteq J .\end{cases}
$$

Note that by Proposition 3.13, $\operatorname{supp}(\lambda) \subseteq J$ iff $\operatorname{supp}(\lambda) \subseteq I$. By Lemma 4.2, $\left(w v_{0}\right)^{I} \neq 0$. Therefore

$$
\begin{aligned}
\pi\left(\exp \left(a_{n}\right) w v_{0}\right) & =\pi\left(\sum_{\lambda \in \Phi_{\iota}} \exp \left(\lambda\left(a_{n}\right)\right)\left(w \cdot v_{0}\right)^{\lambda}\right) \\
& =\pi\left(\sum_{\lambda \in \Phi_{\iota}} \exp \left(-\sum_{\alpha \in \Delta_{\sigma}} n_{\alpha}(\lambda) \alpha\left(a_{n}\right)\right)\left(w v_{0}\right)^{\lambda}\right) \\
& \stackrel{n \rightarrow \infty}{\longrightarrow} \pi\left(\sum_{\lambda: \operatorname{supp}(\lambda) \subset I} \exp \left(-\sum_{\alpha \in \Delta_{\sigma}} n_{\alpha}(\lambda) \alpha(a)\right)\left(w v_{0}\right)^{\lambda}\right) \\
& =\pi\left(\exp (a)\left(w v_{0}\right)^{I}\right) .
\end{aligned}
$$

This shows that $V_{J, w}^{\infty} \subset \pi\left(K \exp \left(\mathfrak{a}^{I,+}\right)\left(w v_{0}\right)^{I}\right)$. On the other hand, given $a \in \mathfrak{a}^{I,+}$, one can find a sequence $\left\{a_{n}\right\} \subset \mathfrak{a}^{+}$such that $\alpha\left(a_{n}\right)=\alpha(a)$ for $\alpha \in I, \alpha\left(a_{n}\right)$ is bounded for $\alpha \in J \backslash I$, and $\alpha\left(a_{n}\right) \rightarrow+\infty$ for $\alpha \in \Delta_{\sigma} \backslash J$. This completes the proof of the first equality.

By Proposition $3.13,\left(w v_{0}\right)^{J}=\left(w v_{0}\right)^{I}$, and using that $\mathfrak{a}^{J,+} \subset \mathfrak{a}_{I}+\mathfrak{a}^{I,+}$, we deduce that

$$
\exp \left(\mathfrak{a}^{J,+}\right)\left(w v_{0}\right)^{I} \subset \mathbb{R}^{+} \cdot \exp \left(\mathfrak{a}^{I,+}\right)\left(w v_{0}\right)^{I} .
$$

This implies the second equality.

The third equality is a consequence of the first two equalities.

Using the same argument as in the proof Proposition 4.13, we also deduce 
Proposition 4.14. For every $w \in \mathcal{W}$ and $J \subset \Delta_{\sigma}$,

$$
\overline{V_{J, w}^{\infty}}=\bigcup_{\lambda_{\iota} \text {-connected } I \subset J} V_{I, w}^{\infty} .
$$

Note that Proposition 4.14implies that the orbit $\mathcal{O}_{I, w}$ is open iff $I \subsetneq \Delta_{\sigma}$ is a maximal $\lambda_{\iota}$-connected set. In this case, $|I|=\left|\Delta_{\sigma}\right|-1$.

4.3. Notation and basic facts. For $g \in G$ let $c_{g}$ denote the inner conjugation by $g$. For $w \in \mathcal{W}$, we define the involutive automorphism $\sigma_{w}:=c_{w} \circ \sigma \circ c_{w}^{-1}$. Since $\theta(w)=w$, and $\sigma$ and $\theta$ commute, we have that $\theta$ and $\sigma_{w}$ also commute. Let

$$
\mathfrak{b}_{0}:=\{X \in \mathfrak{b}: \sigma(X)=X\}=\mathfrak{b} \cap \mathfrak{h}=\mathfrak{b} \cap \mathfrak{a}^{\perp} .
$$

Since $\operatorname{Ad} w(\mathfrak{a})=\mathfrak{a}$ and $\operatorname{Ad} w(\mathfrak{b})=\mathfrak{b}$, and $\operatorname{Ad} w$ preserves the Killing form, we have Ad $w\left(\mathfrak{b}_{0}\right)=\mathfrak{b}_{0}$. Therefore

$$
\sigma_{w}(\mathfrak{b})=\mathfrak{b}, \mathfrak{b}_{0}=\left\{X \in \mathfrak{b}: \sigma_{w}(X)=X\right\}, \mathfrak{a}=\left\{X \in \mathfrak{b}: \sigma_{w}(X)=-X\right\} .
$$

Parabolic subalgebra $\mathfrak{p}_{J}$ and and a decomposition of its Levi subalgebra. Let $J \subset \Delta_{\sigma}$. Since $\sigma_{w}(\alpha)=-\alpha$ for all $\alpha \in \Sigma_{\sigma}$, we have that $\sigma_{w}\left(\mathfrak{a}_{J}\right)=\mathfrak{a}_{J}$. Since $\sigma_{w}$ preserves the Killing form on $\mathfrak{g}$, we have that $\sigma_{w}\left(\mathfrak{a}^{J}\right)=\mathfrak{a}^{J}$. Let $\mathfrak{z}_{\mathfrak{g}}\left(\mathfrak{a}_{J}\right)$ denote the centralizer of $\mathfrak{a}_{J}$ in $\mathfrak{g}$. Let

$$
\Sigma_{J}=\left\{\beta \in \Sigma_{\sigma}: \beta=\sum_{\alpha \in J} n_{\alpha} \alpha, n_{\alpha} \in \mathbb{Z}\right\} \quad \text { and } \quad \Sigma_{J}^{+}=\Sigma_{J} \cap \Sigma_{\sigma}^{+} .
$$

Define

$$
\begin{aligned}
& \mathfrak{n}_{J}:=\sum_{\beta \in \Sigma_{\sigma}^{+} \backslash \Sigma_{J}} \mathfrak{g}_{\beta}, \quad \text { and } \\
& \mathfrak{p}_{J}:=\mathfrak{z}_{\mathfrak{g}}\left(\mathfrak{a}_{J}\right) \oplus \mathfrak{n}_{J},
\end{aligned}
$$

which is a parabolic subalgebra of $\mathfrak{g}$. We define

$$
\begin{aligned}
\mathfrak{m}_{0} & =\left[\mathfrak{g}_{0}, \mathfrak{g}_{0}\right], \quad \text { where } \mathfrak{g}_{0}=\mathfrak{z}_{\mathfrak{g}}(\mathfrak{a}) \text { as before, } \\
\mathfrak{m}_{J} & =\sum_{\beta \in \Sigma_{J}^{+}} \mathfrak{g}_{-\beta}+\left[\mathfrak{g}_{-\beta}, \mathfrak{g}_{\beta}\right]+\mathfrak{g}_{\beta} .
\end{aligned}
$$

Then

$$
\left[\mathfrak{z}_{\mathfrak{g}}\left(\mathfrak{a}_{J}\right), \mathfrak{z}_{\mathfrak{g}}\left(\mathfrak{a}_{J}\right)\right]=\mathfrak{m}_{0}+\mathfrak{m}_{J}
$$

Note that $\left[\mathfrak{m}_{0}, \mathfrak{m}_{J}\right]=\mathfrak{m}_{J}$ and $\left[\mathfrak{m}_{J}, \mathfrak{m}_{J}\right] \subset \mathfrak{m}_{J}$. Since $\left[\mathfrak{z}_{\mathfrak{g}}\left(\mathfrak{a}_{J}\right), \mathfrak{z}_{\mathfrak{g}}\left(\mathfrak{a}_{J}\right)\right]$ is semisimple, its ideal $\mathfrak{m}_{J}$ is semisimple.

Since $\mathfrak{m}_{0}$ is semisimple for the ideal

$$
\mathfrak{m}_{\lambda_{\iota}}:=\left\{x \in \mathfrak{m}_{0}: x W^{\lambda_{\iota}}=0\right\},
$$

there exists an ideal $\mathfrak{m}_{c}$ such that

$$
\mathfrak{m}_{0}=\mathfrak{m}_{c} \oplus \mathfrak{m}_{\lambda_{\iota}} \text {. }
$$

Also, there exist ideals $\mathfrak{m}_{c}^{J}$ and $\mathfrak{m}_{\lambda_{\iota}}^{J}$ of $\mathfrak{m}_{c}$ and $\mathfrak{m}_{\lambda_{\iota}}$ respectively, such that

$$
\mathfrak{m}_{c}+\mathfrak{m}_{J}=\mathfrak{m}_{c}^{J} \oplus \mathfrak{m}_{J} \quad \text { and } \quad \mathfrak{m}_{\lambda_{\iota}}+\mathfrak{m}_{J}=\mathfrak{m}_{\lambda_{\iota}}^{J} \oplus \mathfrak{m}_{J}
$$


By Remark 3.5, $\mathfrak{b}_{0}=\operatorname{span}\left\{b_{\tilde{\delta}}: \tilde{\delta} \in \Delta_{0}\right\} \subset \mathfrak{m}_{0}$. Since $\mathfrak{m}_{0} \subset \mathfrak{g}_{0}$ is semisimple, we have $\mathfrak{a} \cap \mathfrak{m}_{0}=\{0\}$. Therefore

$$
\mathfrak{b}_{0}=\mathfrak{m}_{0} \cap \mathfrak{b} .
$$

Since $\sigma_{w}\left(\mathfrak{g}_{\beta}\right)=\mathfrak{g}_{-\beta}$, we conclude that $\mathfrak{m}_{J}$ is $\sigma_{w}$-invariant. Similarly, $\mathfrak{m}_{J}$ is $\theta$ invariant. Therefore

$$
\mathfrak{m}_{J}=\left(\mathfrak{k} \cap \mathfrak{m}_{J}\right) \oplus\left(\mathfrak{p} \cap(\operatorname{Ad} w) \mathfrak{q} \cap \mathfrak{m}_{J}\right) \oplus\left((\operatorname{Ad} w) \mathfrak{h} \cap \mathfrak{m}_{J}\right) .
$$

Note that $\mathfrak{a}$ is a maximal abelian subalgebra of $\mathfrak{p} \cap(\operatorname{Ad} w) \mathfrak{q}$. Next we show that

$$
\mathfrak{a} \cap \mathfrak{m}_{J}=\mathfrak{a}^{J} .
$$

For each $\beta \in J$, let $a_{\beta} \in \mathfrak{a}$ be such that $\left\langle a_{\beta}, a\right\rangle=\beta(a)$ for all $a \in \mathfrak{a}$. Then $\left\{a_{\beta}: \beta \in J\right\}$ is a basis of $\mathfrak{a}^{J}$. Hence by Remark 3.9 and (4.20), we have $\mathfrak{a}^{J} \subset \mathfrak{m}_{J}$. For any $\beta \in \Sigma_{J}^{+}$, if $Y_{ \pm} \in \mathfrak{g}_{ \pm \beta}$, and $X \in \mathfrak{a}_{J}$, then

$$
\left\langle X,\left[Y_{-}, Y_{+}\right]\right\rangle=\left\langle\left[X, Y_{-}\right], Y_{+}\right\rangle=-\beta(X)\left\langle Y_{-}, Y_{+}\right\rangle=0 .
$$

Therefore, by (4.20), $\left(\mathfrak{a} \cap \mathfrak{m}_{J}\right) \perp \mathfrak{a}_{J}$; that is, $\mathfrak{a} \cap \mathfrak{m}_{J} \subset \mathfrak{a}^{J}$. This justifies (4.24).

Note that center of $\mathfrak{z}_{\mathfrak{g}}\left(\mathfrak{a}_{J}\right)$ is contained in the center of $\mathfrak{z}_{\mathfrak{g}}(\mathfrak{b})$, which in turn is contained in $\mathfrak{c}=(\mathfrak{k} \cap \mathfrak{c})+\mathfrak{b}$. As $\mathfrak{b}=\mathfrak{a}+\mathfrak{b}_{0}$, for $\mathfrak{c}_{J}:=\operatorname{Center}\left(\mathfrak{z}_{\mathfrak{g}}(\mathfrak{a} J)\right) \cap \mathfrak{k}$,

$$
\mathfrak{z} \mathfrak{g}\left(\mathfrak{a}_{J}\right)=\mathfrak{c}_{J}+\mathfrak{b}+\mathfrak{m}_{0}+\mathfrak{m}_{J}=\mathfrak{c}_{J} \oplus \mathfrak{a}_{J} \oplus \mathfrak{m}_{c}^{J} \oplus \mathfrak{m}_{\lambda_{\iota}}^{J} \oplus \mathfrak{m}_{J}
$$

Let $P_{J}$ denote the parabolic subgroup of $G$ associated to $\mathfrak{p}_{J}$. Let $M_{J}$ and $N_{J}$ denote the analytic subgroups of $P_{J}$ associated to the subalgebras $\mathfrak{m}_{J}$ and $\mathfrak{n}_{J}$, respectively. In the course of the above discussion, we have also proved the following:

Proposition 4.26. Let $J \subset \Delta_{\sigma}$ and $w \in \mathcal{W}$. Then the semisimple group $M_{J}$ is invariant under $\theta$ and $\sigma_{w}$, and $\mathfrak{a}^{J}$ is a Cartan subalgebra of $M_{J}$ for the pair $\left(\theta, \sigma_{w}\right)$.

Note that $\mathfrak{a}^{J}$ has the system of simple roots $J$ with the Weyl group $\mathcal{W}_{J}$, and we have the decomposition

$$
M_{J}=\left(M_{J} \cap K\right) \exp \left(\mathfrak{a}^{J,+}\right) \mathcal{W}_{J}\left(w H w^{-1} \cap M_{J}\right) .
$$

Lemma 4.28. Let $J \subset \Delta_{\sigma}$. Then the following assertions hold:

(i) $W^{J}=\left\{v \in W: a v=\lambda_{\iota}(a) v, \forall a \in \mathfrak{a}_{J}\right\}$.

(ii) $Z_{G}\left(A_{J}\right) \cdot W^{J} \subset W^{J}$.

(iii) $\left(Z_{G}\left(A_{J}\right) \cap w H w^{-1}\right)\left(w v_{0}\right)^{J}=\left(w v_{0}\right)^{J}$ for any $w \in \mathcal{W}$.

(iv) $N_{J}$ acts trivially on $W^{J}$.

Proof. If $\lambda \in \Phi_{\iota}$ such that $\operatorname{supp} \lambda \subset J$ and $a \in \mathfrak{a}_{J}$, then by definition of $\operatorname{supp} \lambda$, we have that $\lambda(a)=\lambda_{\iota}(a)$. Therefore $a v=\lambda_{\iota}(a) v$ for all $v \in W^{\lambda}$.

Since an open subset of $\mathfrak{a}_{J}$ is contained in the boundary of $\mathfrak{a}^{+}$, there exits $X \in \mathfrak{a}_{J}$ such that $\alpha(X)>0$ for all $\alpha \in \Delta_{\sigma} \backslash J$. Therefore if $\lambda \in \Phi_{\iota}$ such that $\operatorname{supp} \lambda \not \subset J$, then $\lambda(X)<\lambda_{\iota}(X)$. Therefore (i) follows from the above two observations and the definition of $W^{J}$.

Since the centralizer preserves the isotypical components, we obtain (ii).

Let $X \in \mathfrak{a}_{J}$ be as above. Then

$$
\lim _{t \rightarrow \infty} e^{-t \lambda_{\iota}(X)} \exp (t X)\left(w v_{0}\right)=\left(w v_{0}\right)^{J} .
$$


Since $Z_{G}\left(A_{J}\right) \cap w H w^{-1}$ fixes $w v_{0}$, it acts trivially on the $\mathbb{R}$-span of $A_{J}\left(w v_{0}\right)$, which contains $\left(w v_{0}\right)^{J}$ by (4.29). Therefore (iii) holds.

Let $\lambda \in \Phi_{\iota}$ be such that $\operatorname{supp} \lambda \subset J$; that is, $\lambda=\lambda_{\iota}-\sum_{\alpha \in J} n_{\alpha} \alpha$, where all $n_{\alpha} \geq 0$. Suppose that $\gamma=\sum_{\alpha \in \Delta_{\sigma}} m_{\alpha} \alpha$, where all $m_{\alpha} \geq 0$, is such that $\lambda+\gamma \in \Phi_{\iota}$. Since $\lambda_{\iota}$ is the highest weight in $\Phi_{\iota}$, we have

$$
\lambda_{\iota}-(\lambda+\gamma) \in \Sigma_{\sigma}^{+}
$$

Then $m_{\alpha}=0$ for all $\alpha \in \Delta_{\sigma} \backslash J$; that is, $\gamma \in \Sigma_{J}^{+}$. This shows that if $\gamma \in \Sigma_{\sigma}^{+} \backslash \Sigma_{J}$, then

$$
\mathfrak{g}_{\gamma} W^{\lambda} \subset W^{\lambda+\gamma}=0 .
$$

Therefore $\mathfrak{n}_{J} W^{J}=0$. Thus (iv) holds.

Proposition 4.30. $\mathfrak{z}_{J}:=\mathfrak{z}_{\mathfrak{g}}\left(\mathfrak{a}_{J}\right)$ acts irreducibly on $W^{J}$.

Proof. With notation as in the proof of Lemma 4.1, we have $U_{0}(\mathfrak{n}) W^{\lambda_{\iota}}=0$ and $U_{0}\left(\mathfrak{n}^{-}\right) W^{\lambda_{\iota}} \subset W^{\prime}$. This easily implies that $\mathfrak{g}_{0}$ acts irreducibly on $W^{\lambda_{\iota}}$.

Since $\mathfrak{n}=\left(\mathfrak{m}_{J} \cap \mathfrak{n}\right)+\mathfrak{n}_{J}$, and $\mathfrak{n}_{J} \cdot W^{J}=0$, it follows from Lemma 4.1

$$
W^{\lambda_{\iota}}=\left\{v \in W^{J}:\left(\mathfrak{m}_{J} \cap \mathfrak{n}\right) v=0\right\} .
$$

Since $\mathfrak{z}_{J}$ is reductive, $W^{J}$ is a direct sum of irreducible $\mathfrak{z}_{J}$-modules, and each of them admits a nonzero subspace which is annihilated by $\mathfrak{m}_{J} \cap \mathfrak{n}$ (Engel's theorem). Hence by (4.31) each of the $\mathfrak{z} J$-submodules contains a nonzero subspace of $W^{\lambda_{\iota}}$ which in turn is invariant under $\mathfrak{g}_{0} \subset \mathfrak{z} J$. Since $\mathfrak{g}_{0}$ acts irreducibly on $W^{\lambda_{\iota}}$, we conclude that $\mathfrak{z} J$ acts irreducibly on $W^{J}$.

Lemma 4.32. $\mathfrak{m}_{c} \subset \mathfrak{k} \cap \mathfrak{z}_{\mathfrak{g}}(\mathfrak{b})$.

Proof. Let $\tilde{\mathfrak{m}}_{0}$ be maximal noncompact ideal of $\mathfrak{m}_{0}$. It follows from [Sc, Lemma 7.1.4] that $\tilde{\mathfrak{m}}_{0} \subset(\operatorname{Ad} w)(\mathfrak{h})$. Hence, by Lemma $4.28\left(\right.$ iii), $\tilde{\mathfrak{m}}_{0} \subset \mathfrak{m}_{\lambda_{\iota}}$. Since by Remark 3.5, $\mathfrak{b}_{0}=\operatorname{span}\left\{b_{\tilde{\delta}}: \tilde{\delta} \in \Delta_{0}\right\} \subset \tilde{\mathfrak{m}}_{0}$, we deduce that $\mathfrak{m}_{c} \subset \mathfrak{z}_{\mathfrak{g}}(\mathfrak{b})$. This implies the lemma.

Definition 4.33. For a $\lambda_{\iota}$-connected $I$ of $\Delta_{\sigma}$, let $I^{\prime}=\left(I \cup\left\{\lambda_{\iota}\right\}\right)^{\perp} \cap \Delta_{\sigma}$; that is, the set of roots in $\Delta_{\sigma}$ which are orthogonal to $\lambda_{\iota}$ and all roots in $I$. We define

$$
J(I):=I \cup I^{\prime} .
$$

We note the following:

(1) $J(I)$ uniquely determines $I$, as $I$ is the maximal $\lambda_{\iota}$-connected subset of $J(I)$.

(2) If $\beta, \gamma \in \Sigma_{\sigma}^{+}$and $\beta \perp \gamma$, then $\beta+\gamma \notin \Sigma_{\sigma}^{+}$. Therefore $\left[\mathfrak{m}_{I}, \mathfrak{m}_{I^{\prime}}\right]=0$, and hence $M_{J(I)}=M_{I} M_{I^{\prime}}$ is an almost direct product. Also $\Sigma_{J(I)}=\Sigma_{I} \cup \Sigma_{I^{\prime}}$.

(3) $W^{J(I)}=W^{I}$.

Proposition 4.34. Let $I$ and $J=J(I)$ be as above. Then

$$
\left(\mathfrak{m}_{\lambda_{\iota}}^{J}+\mathfrak{m}_{I^{\prime}}\right) W^{I}=0 \quad \text { and } \quad c w=\Lambda_{\iota}(c) w, \quad \forall c \in \mathfrak{c}_{J}, w \in W^{I} .
$$

The Lie algebra $\mathfrak{m}_{c}^{I} \oplus \mathfrak{m}_{I}$ acts irreducibly and faithfully on $W^{I}$ over $\mathbb{K}$. 
Proof. By the definition of $I^{\prime}$ and Proposition [3.13, for any $\gamma \in \Sigma_{I^{\prime}}$ and $\lambda \in \Phi_{\iota}$ with $\operatorname{supp} \lambda \subset I$, we have $\lambda+\gamma \notin \Phi_{\iota}$. Therefore, $\mathfrak{m}_{I^{\prime}} W^{J}=0$. Since $\mathfrak{m}_{\lambda_{\iota}}^{J}$ is a semisimple ideal in $\mathfrak{z}_{\mathfrak{g}}\left(\mathfrak{a}_{J}\right)$, by Proposition 4.30 and (4.21), we conclude that $\mathfrak{m}_{\lambda_{\iota}}^{J} W^{I}=0$. By Proposition 4.30 and (4.25), $\mathfrak{c}_{J} \oplus \mathfrak{m}_{c}^{J} \oplus \mathfrak{m}_{I}$ acts irreducibly on $W^{J}$ over $\mathbb{K}$. If $\mathbb{K}=\mathbb{R}$ then $W_{\mathbb{C}}^{\Lambda_{\iota}} \cap W$ is a one-dimensional $\mathfrak{c}_{J}$-invariant subspace. Since $\exp \left(\mathfrak{c}_{J}\right) \subset K$, we conclude that $\mathfrak{c}_{J}\left(W_{\mathbb{C}}^{\Lambda_{\iota}} \cap W\right)=0$. Hence, by irreducibility, $\mathfrak{c}_{J} W^{J}=0$. Suppose if $\mathbb{K}=\mathbb{C}$, then $\mathfrak{c}_{J}$ being central in $\mathfrak{c}_{J} \oplus \mathfrak{m}_{c}^{J} \oplus \mathfrak{m}_{I}$, by the irreducibility we conclude that $\mathfrak{c}_{J}$ acts via $\mathbb{K}$-scalars on $W^{J}$. This proves the first claim.

It follows from above that $\mathfrak{m}_{c}^{J} \oplus \mathfrak{m}_{I}$ act irreducibly on $W^{J}$ over $\mathbb{K}$. Since $\mathfrak{m}_{0} \cap \mathfrak{m}_{I^{\prime}} \subset$ $\mathfrak{m}_{\lambda_{\iota}}$, we have that $\mathfrak{m}_{c}^{J}=\mathfrak{m}_{c}^{I}$. This proves irreducibility.

By (4.21) and (4.22), $\mathfrak{m}_{c}^{I}$ acts faithfully on $W^{\lambda_{\iota}}$, and hence on $W^{I}$. We observe that any nonzero ideal of $\mathfrak{m}_{I}$ contains $a^{I_{1}}$ for some $\emptyset \neq I_{1} \subset I$ such that $I_{1} \perp\left(I \backslash I_{1}\right)$. Since $I$ is $\lambda_{\iota}$-connected, $I_{1}$ is $\lambda_{\iota}$-connected, and hence $\mathfrak{a}^{I_{1}} \not \subset \operatorname{ker}\left(\lambda_{\iota}\right)$. But then $\mathfrak{a}^{I_{1}} v_{0}^{\lambda_{\iota}}=$ $\lambda_{\iota}\left(\mathfrak{a}^{I_{1}}\right) v_{0}^{\lambda_{\iota}} \neq 0$. Thus, $\mathfrak{m}_{I}$ acts faithfully on $W^{I}$.

Proposition 4.35. For a $\lambda_{\iota}$-connected subset $I$ of $\Delta_{\sigma}$ and $w \in \mathcal{W}$, we have

$$
\pi\left(G\left(w v_{0}\right)^{I}\right)=\pi\left(K \exp \left(\mathfrak{a}^{I,+}\right) \mathcal{W}_{I}\left(w v_{0}\right)^{I}\right)=\pi\left(K \exp \left(\mathfrak{a}^{I,+}\right)\left(\mathcal{W}_{I} w v_{0}\right)^{I}\right) .
$$

Proof. By the Iwasawa decomposition we have $G=K P_{I}=K \exp \left(\mathfrak{z}_{\mathfrak{g}}\left(\mathfrak{a}_{I}\right)\right)\left(\mathfrak{a}_{I}\right) N_{I}$. Now $N_{I}$ acts trivially on $W^{I}$. Therefore, in view of Proposition 4.34,

$$
\pi\left(G\left(w v_{0}\right)^{I}\right)=\pi\left(K M_{I}\left(w v_{0}\right)^{I}\right) .
$$

Now the first equality follows from Lemma 4.28(iii), and (4.27).

Since the weight spaces $W^{\lambda}, \lambda \in \Phi_{\iota}$, are orthogonal with respect to a $K$-invariant scalar product, and by Lemma 4.28, $\mathcal{W}_{I} \subset M_{I} \cap K$ preserves $W^{I}$, it follows that $\mathcal{W}_{I}$ preserves the orthogonal complement of $W^{I}$, and hence

$$
w \cdot v^{I}=(w v)^{I} \quad \text { for all } w \in \mathcal{W}_{I} \text { and } v \in W .
$$

This justifies the second equality in the proposition.

4.4. Disjointness of the $G$-orbits in the boundary and stabilizers of $\left(w_{0} v_{0}\right)^{I}$.

Lemma 4.36. Let $I$ be a $\lambda_{\iota}$-connected subset of $\Delta_{\sigma}$ and let $J=J(I)$. Then

$$
\operatorname{Stab}_{G}\left(W^{I}\right):=\left\{g \in G: g W^{I}=W^{I}\right\}=P_{J} .
$$

Proof. Let $Q=\operatorname{Stab}_{G}\left(W^{I}\right)$. It follows from Lemma 4.28 that $Q \supset P_{J}$. Hence, $Q=P_{S}$ with $J \subset S \subset \Delta_{\sigma}$. Since $\mathfrak{z}_{S}:=\mathfrak{z}_{\mathfrak{g}}\left(\mathfrak{a}_{S}\right) \subset P_{S}$, we have $\mathfrak{z}_{S} W^{I}=W^{I}$. By Proposition 4.30 $\mathfrak{z} S$ acts irreducibly on $W^{S}$. Therefore $W^{I}=W^{S}$. Since $I$ is $\lambda_{\iota}$-connected, by Proposition $3.13 I$ is the maximal $\lambda_{\iota}$-connected component of $S$. Hence by Definition 4.33 $S \subset J(I)$. Hence $J=S$.

Let $I$ be a $\lambda_{\iota}$-connected subset of $\Delta_{\sigma}, J=J(I)$, and $w_{0} \in \mathcal{W}$. We consider the group $L=\left\{g \in G: g\left(w_{0} v_{0}\right)^{I}=\left(w_{0} v_{0}\right)^{I}\right\}$ with the Lie algebra $\mathfrak{l}=\left\{X \in \mathfrak{g}: X\left(w_{0} v_{0}\right)^{I}=\right.$ $0\}$. Note that $\mathfrak{a}_{J}$ normalizes $\mathfrak{l}$, because $\mathfrak{a}_{J} \subset \mathfrak{a}_{I}$, and by Lemma $4.28\left(w_{0} v_{0}\right)^{I}$ is an eigenvector for each element of $\mathfrak{a}_{I}$. Moreover by Lemma 4.28, we have $\mathfrak{n}_{J} \subset \mathfrak{l}$. Therefore

$$
\mathfrak{l}=\mathfrak{n}_{J}+\mathfrak{l} \cap \mathfrak{z}_{\mathfrak{g}}\left(\mathfrak{a}_{J}\right)+\mathfrak{l} \cap \sum_{\beta \in \Sigma_{\sigma}^{+} \backslash \Sigma_{J}} \mathfrak{g}_{-\beta} .
$$


By (4.25) and Proposition 4.34

$$
\mathfrak{l} \cap \mathfrak{z}_{\mathfrak{g}}\left(\mathfrak{a}_{J}\right)=\left(\mathfrak{c}_{J} \cap \operatorname{ker} \Lambda_{\iota}\right) \oplus\left(\mathfrak{a}_{J} \cap \operatorname{ker} \lambda_{\iota}\right) \oplus \mathfrak{m}_{\lambda_{\iota}}^{J} \oplus \mathfrak{m}_{I^{\prime}} \oplus\left(\left(\mathfrak{m}_{c}^{I} \oplus \mathfrak{m}_{I}\right) \cap \mathfrak{l}\right) .
$$

Proposition 4.39. We have

$$
\left(\mathfrak{m}_{c}^{I} \oplus \mathfrak{m}_{I}\right) \cap \mathfrak{l}=\left(\mathfrak{m}_{c}^{I} \oplus \mathfrak{m}_{I}\right) \cap\left(\operatorname{Ad} w_{0}\right) \mathfrak{h}=\left(\mathfrak{m}_{c}^{I} \cap\left(\operatorname{Ad} w_{0}\right) \mathfrak{h}\right) \oplus\left(\mathfrak{m}_{I} \cap\left(\operatorname{Ad} w_{0}\right) \mathfrak{h}\right) .
$$

In particular, the orthogonal projection of $\mathfrak{l}$ on $\mathfrak{a}^{I}$ is trivial.

Proof. By Proposition 4.26, applied to $I$ in place of $J$ and $w_{0}$ in place of $w, \mathfrak{h}_{I, w_{0}}:=$ $\left(\mathfrak{m}_{c}^{I}+\mathfrak{m}_{I}\right) \cap \operatorname{Ad} w_{0}(\mathfrak{h})$ is a symmetric subalgebra of $\mathfrak{m}_{c}^{I}+\mathfrak{m}_{I}$. By Lemma 4.28(iii), $\mathfrak{h}_{I, w_{0}}\left(w_{0} v_{0}\right)^{I}=0$. Let $M_{c}^{J}$ denote the analytic subgroup of $G$ associated to $\mathfrak{m}_{c}^{I}$. Due to Proposition 4.34, we can apply Corollary 4.7 to $M_{c}^{I} M_{I}$ in place of $G$, and $W^{I}$ in place of $E$, to obtain the first equality. The second equality holds because $\mathfrak{m}_{c}^{I}$ and $\mathfrak{m}_{I}$ are invariant under $\sigma$. The last conclusion follows from Proposition 4.26 because $\mathfrak{a}^{I}$ is orthogonal to $\operatorname{Ad} w_{0}(\mathfrak{h})$.

Proposition 4.40. $\mathfrak{l} \subset \mathfrak{p}_{J}$.

Proof. Suppose that $\mathfrak{l} \not \subset \mathfrak{p}_{J}$. Then by (4.37) there exists $\beta \in \Sigma_{\sigma}^{+} \backslash \Sigma_{J}, \tilde{\beta} \in \Sigma^{+}$with $\left.\tilde{\beta}\right|_{\mathfrak{a}}=\beta$, and $0 \neq X \in \mathfrak{l}$ such that

$$
X=X_{-\tilde{\beta}}+Y \quad \text { where } 0 \neq X_{-\tilde{\beta}} \in \mathfrak{g}_{-\tilde{\beta}}(\mathfrak{b}) \text { and } Y \in \sum_{\tilde{\gamma} \in \Sigma \backslash\{0\}:\left.\tilde{\gamma}\right|_{\mathfrak{a}_{J}}=0} \mathfrak{g}_{-\tilde{\beta}+\tilde{\gamma}} .
$$

Replacing $X$ by a scalar multiple from the beginning, without loss of generality we may assume that $B\left(X_{-\tilde{\beta}}, X_{-\tilde{\beta}}\right)=1$ (see (3.3) $)$. Since $-\theta\left(X_{\tilde{\beta}}\right) \in \mathfrak{g}_{\tilde{\beta}}(\mathfrak{b}) \subset \mathfrak{g}_{\beta} \subset \mathfrak{l}$, we have $\left[-\theta\left(X_{-\tilde{\beta}}\right), X\right]=b_{\tilde{\beta}}+Z \in \mathfrak{l}$, where

$$
b_{\tilde{\beta}}=\left[-\theta\left(X_{-\tilde{\beta}}\right), X_{-\tilde{\beta}}\right] \in \mathfrak{b} \quad \text { and } \quad Z=\left[-\theta\left(X_{-\tilde{\beta}}\right), Y\right] \in \sum_{\tilde{\gamma} \in \Sigma \backslash\{0\}} \mathfrak{g}_{\tilde{\gamma}}(\mathfrak{b}) \cap \mathfrak{z}_{\mathfrak{g}}\left(\mathfrak{a}_{J}\right) .
$$

Therefore $b_{\tilde{\beta}}+Z \in \mathfrak{l} \cap \mathfrak{p}_{J}$ and its projection on $\mathfrak{b}$ equals $b_{\tilde{\beta}}$. By (4.37), (4.38) and Proposition 4.39 ,

$$
b_{\tilde{\beta}} \in \mathfrak{b}_{0}+\left(\mathfrak{a}_{I} \cap \operatorname{ker} \lambda_{\iota}\right) .
$$

If $a_{\tilde{\beta}}$ is the projection of $b_{\tilde{\beta}}$ on $\mathfrak{a}$, then

$$
a_{\tilde{\beta}} \in \mathfrak{a}_{I} \cap \operatorname{ker} \lambda_{\iota} .
$$

Since $\mathfrak{b}_{0} \perp \mathfrak{a}$, by Remark 3.5, for all $a \in \mathfrak{a}$,

$$
\left\langle a, a_{\tilde{\beta}}\right\rangle=\left\langle a, b_{\tilde{\beta}}\right\rangle=\tilde{\beta}(a)=\beta(a) .
$$

Therefore, $\beta \perp\left(I \cup\left\{\lambda_{\iota}\right\}\right)$. Using that scalar products of simple roots are nonpositive, we deduce that $\beta \in\left\langle I^{\prime}\right\rangle \subset J$, which is a contradiction.

From (4.37), (4.38), Proposition 4.39 and Proposition 4.40 we deduce the following:

Corollary 4.41. We have

$\mathfrak{l}=\left(\mathfrak{c}_{J} \cap \operatorname{ker} \Lambda_{\iota}\right) \oplus\left(\mathfrak{a}_{J} \cap \operatorname{ker} \lambda_{\iota}\right) \oplus \mathfrak{m}_{\lambda_{\iota}}^{J} \oplus \mathfrak{m}_{I^{\prime}} \oplus\left(\mathfrak{m}_{c}^{J} \cap \operatorname{Ad} w_{0}(\mathfrak{h})\right) \oplus\left(\mathfrak{m}_{I} \cap \operatorname{Ad} w_{0}(\mathfrak{h})\right) \oplus \mathfrak{n}_{J}$. 
In particular,

$$
\text { Unipotent Radical }(L)=N_{J} \quad \text { and } \quad L \subset P_{J}
$$

Proof of Theorem 4.10. Consider a divergent sequence

$$
v_{n}=k_{n} \exp \left(a_{n}\right) w_{n} v_{0} \in V
$$

where $k_{n} \in K, a_{n} \in \mathfrak{a}^{+}, w_{n} \in \mathcal{W}$. After passing to a subsequence if necessary, we can assume that $k_{n} \rightarrow k \in K, w_{n}=w \in \mathcal{W}$, and that there exists $J \subsetneq \Delta_{\sigma}$ such that $\alpha\left(a_{n}\right)$ is bounded for $\alpha \in J$ and $\alpha\left(a_{n}\right) \rightarrow \infty$ for $\alpha \in \Delta_{\sigma} \backslash J$. Then the limit points of the sequence $v_{n}$ are in $V_{J, w}^{\infty}$. This proves that

$$
V^{\infty}=\bigcup_{J \subsetneq \Delta_{\sigma}, w \in \mathcal{W}} V_{J, w}^{\infty} .
$$

Moreover, by Proposition 4.13, it suffices to take the union over $\lambda_{\iota}$-connected subsets only. Then (4.11) follows from Proposition 4.35.

By Corollary 4.41 the unipotent radical of the stabilizer of $g_{i}\left(w_{i} v_{0}\right)^{I_{i}}$ is $g_{i} N_{J_{i}} g_{i}^{-1}$. Therefore if the $G$-orbits of $\left(w_{i} v_{0}\right)^{I_{i}}$ are same, then $g_{1} N_{J_{1}} g_{1}^{-1}=g_{2} N_{J_{2}} g_{2}^{-1}$. Since $P_{J_{i}}=N_{G}\left(N_{J_{i}}\right)$, we have $g_{1} P_{J_{1}} g_{1}^{-1}=g_{2} P_{J_{2}} g_{2}^{-1}$. Hence $J_{1}=J_{2}$ and $I_{1}=I_{2}$. Thus (4.12) follows.

Note that Theorem 1.22 follows from Theorem 4.10, Proposition 4.13 and Proposition 4.14.

Recall that for $\Omega \subset S(W)$, we have defined

$$
\Theta_{\Omega}=\left\{I \subset \Delta_{\sigma}: I \text { is } \lambda_{\iota} \text {-connected and } \bar{\Omega} \cap V_{I}^{\infty} \neq \emptyset\right\} .
$$

We denote by $\Theta_{\Omega}^{\min }$ the set of minimal elements in $\Theta_{\Omega}$ with respect to inclusion.

Corollary 4.42 (Tube lemma). For any compact set $\Omega \subset S(W)$, there exists a collection $\left\{U_{J} \subset \mathfrak{a}^{J,+}: J \in \Theta_{\Omega}^{\min }\right\}$ of compact sets such that

$$
\Omega \cap V_{I}^{\infty} \subset \bigcup_{J \in \Theta_{\Omega}^{\min }} \pi\left(K \exp \left(U_{J}+\mathfrak{a}_{J}^{+}\right)\left(\mathcal{W} v_{0}\right)^{I}\right) \quad \text { for every } I \subseteq \Delta_{\sigma} .
$$

Proof. Suppose that the corollary fails. Then for any choice of compact sets $U_{J}$, there exists $v=\pi\left(k \exp (a)\left(w v_{0}\right)^{I}\right) \in \Omega$ with $k \in K, w \in W$, and $a \in \mathfrak{a}^{+}, a \notin U_{J}+\mathfrak{a}_{J}^{+}$for every $J \in \Theta_{\Omega}^{\min }$. Therefore, there exists $v_{n}=\pi\left(k_{n} \exp \left(a_{n}\right)\left(w_{n} v_{0}\right)^{I}\right) \in \Omega$ with $k_{n} \in K$, $a_{n} \in \mathfrak{a}^{+}, w_{n} \in W$ such that for every $J \in \Theta_{\Omega}^{\min }, \alpha\left(a_{n}\right) \rightarrow \infty$ for at least one $\alpha$ in $J$. Passing to a subsequence, we may assume that for some $I \subset \Delta_{\sigma}, \alpha\left(a_{n}\right) \rightarrow \infty$ if $\alpha \in \Delta_{\sigma} \backslash I$ and $\alpha\left(a_{n}\right)$ is bounded if $\alpha \in I$. Then by Proposition 4.13, the limit points of the sequence $\left\{v_{n}\right\}$ are in $V_{I_{0}, w_{0}}^{\infty}, w_{0} \in \mathcal{W}$, where $I_{0}$ is the largest $\lambda_{\iota}$-connected subset of $I$. Then $\Omega \cap V_{I_{0}}^{\infty} \neq \emptyset$ and $I_{0} \in \Theta_{\Omega}$. On the other hand, $I_{0} \nsupseteq J$ for every $J \in \Theta_{\Omega}^{\min }$. This gives a contradiction and proves the corollary. 


\section{INVARIANT MEASURES AT INFINITY}

In the previous section, we have shown that

$$
V^{\infty}=\bigsqcup_{\lambda_{\iota} \text {-connected } I \subsetneq \Delta_{\sigma}} V_{I}^{\infty}
$$

where

$$
\mathbb{R}^{+} \cdot V_{I}^{\infty}=\bigcup_{w \in \mathcal{W}} G\left(w v_{0}\right)^{I}
$$

In this section we describe an algebraic condition on $I$ so that $V_{I}^{\infty}$ admits a $G$-invariant measure, and give a formula for the measure. We also provide a natural class of $I$ for which the condition holds. The results of this section are obtained mainly for the the sake of more complete description of the boundary. They are not essential for the proofs of the main results stated in the introduction.

Theorem 5.1. Let $I$ be a $\lambda_{\iota}$-connected subset of $\Delta_{\sigma}$. Then for any $w_{0} \in \mathcal{W}$, there exists a $G$-invariant measure on $G\left(w_{0} v_{0}\right)^{I}$ if and only if

$$
\mathfrak{a}_{J} \cap \operatorname{ker} \rho=\mathfrak{a}_{J} \cap \operatorname{ker} \lambda_{\iota},
$$

where $J=J(I)$ is as in Definition 4.33 .

If (5.2) hold, then the $G$-invariant measure on $G\left(w_{0} v_{0}\right)^{I}$, say $\nu_{I, w_{0}}$, is given by (up to a constant multiple)

$$
\begin{aligned}
& \int_{W} f d \nu_{I, w_{0}} \\
& =\int_{K} d k \int_{a \in \mathfrak{a}^{I,+}} d a \int_{\bar{b} \in \mathfrak{a}_{J} / \mathfrak{a}_{J} \cap \operatorname{ker} \lambda_{\iota}} \sum_{w \in \mathcal{W}_{I}} f\left(k \exp (a+b) w\left(w_{0} v_{0}\right)^{I}\right) \delta_{I}(a) e^{2 \rho(b)} d \bar{b}
\end{aligned}
$$

for all $f \in C_{c}\left(G\left(w_{0} v_{0}\right)^{I}\right)$, where $d k, d a$ and $d \bar{b}$ denote the Haar integrals on $K, \mathfrak{a}^{I}$, and $\mathfrak{a}_{J} / \mathfrak{a}_{J} \cap \operatorname{ker} \rho$, respectively, and

$$
\delta_{I}(a):=\prod_{\alpha \in \Sigma_{I}^{+}}(\sinh \alpha(a))^{l_{\alpha}^{+}}(\cosh \alpha(a))^{l_{\alpha}^{-}}, \forall a \in \mathfrak{a}^{I} .
$$

Proof. Let $w_{0} \in \mathcal{W}$. Since $G$ admits no nontrivial positive real characters, there exists a $G$-invariant measure on $G \cdot\left(w_{0} v_{0}\right)^{I}$ if and only if $L:=\operatorname{Stab}_{G}\left(\left(w_{0} v_{0}\right)^{I}\right)$ is unimodular. By Corollary 4.41, $N_{J}$ is the unipotent radical of $L$. Therefore $L$ is unimodular if and only if $\left|\operatorname{det}\left(\left.\operatorname{Ad} g\right|_{\mathfrak{n}_{J}}\right)\right|=1$ for all $g \in L$, if and only if $\operatorname{tr}\left(\left.\operatorname{ad}(x)\right|_{\mathfrak{n}_{J}}\right)=0$ for all $x \in \mathfrak{l}$.

Note that $\mathfrak{c}_{J} \subset \mathfrak{k}$, and $\mathfrak{m}_{c}^{J} \oplus \mathfrak{m}_{\lambda_{\iota}}^{J} \oplus \mathfrak{m}_{J}$ is semisimple. Also each of them normalizes $\mathfrak{n}_{J}$. Therefore

$$
\operatorname{tr}\left(\left.(\operatorname{ad} x)\right|_{\mathfrak{n}_{J}}\right)=0 \quad \text { for all } x \in \mathfrak{c}_{J}+\mathfrak{m}_{c}^{J}+\mathfrak{m}_{\lambda_{\iota}}^{J}+\mathfrak{m}_{J} .
$$

Therefore by Corollary 4.41, $L$ is unimodular if and only if

$$
2 \rho(b)=\sum_{\alpha \in \Sigma_{\sigma}^{+} \backslash\langle J\rangle} \operatorname{tr}\left(\left.\operatorname{ad}(b)\right|_{\mathfrak{g}_{\alpha}}\right)=0, \quad \forall b \in \mathfrak{a}_{J} \cap \operatorname{ker} \lambda_{\iota}
$$


This equation is equivalent to ker $\lambda_{\iota} \cap \mathfrak{a}_{J} \subset \operatorname{ker} \rho \cap \mathfrak{a}_{J}$. If $\mathfrak{a}_{J} \neq\{0\}$, then $\mathfrak{a}_{J} \not \subset \operatorname{ker} \rho$, and hence $\operatorname{ker} \rho \cap \mathfrak{a}_{J}$ is of codimension 1 in $\mathfrak{a}_{J}$. Also ker $\lambda_{\iota} \cap \mathfrak{a}_{J}$ is of codimension at most one in $\mathfrak{a}_{J}$. Therefore (5.5) is equivalent to (5.2). This proves the first part of the theorem.

Now to obtain the formula for the Haar integral on $G$, we suppose that (5.2) holds. In view of (4.18) and (4.25), let $\tilde{M}_{J}$ be the closed subgroup of $P_{J}$ associated to the Lie subalgebra $\mathfrak{c}_{J}+\mathfrak{m}_{c}^{J}+\mathfrak{m}_{\lambda_{\iota}}^{J}+\mathfrak{m}_{J}$ such that $P_{J}=\tilde{M}_{J} A_{J} N_{J}$, where $Z_{G}\left(A_{J}\right)=\tilde{M}_{J} A_{J}$ is a direct product. A right Haar integral on $P_{J}$ can be given by

$$
f \mapsto \int_{\tilde{M}_{J}} d m \int_{\mathfrak{a}_{J}} d b \int_{N_{J}} f(m \exp (b) n) e^{2 \rho(b)} d n, \quad \forall f \in C_{c}\left(P_{J}\right),
$$

where $d m$ and $d n$ denote Haar integrals on $\tilde{M}_{J}$ and $N_{J}$, respectively, and $d b$ denotes the Lebesgue integral on $\mathfrak{a}_{J}$.

Note that

$$
\tilde{M}_{J}=\left(K \cap P_{J}\right) \tilde{M}_{J}^{0}=\left(K \cap P_{J}\right) M_{\lambda_{\iota}}^{J} M_{J},
$$

where $M_{\lambda_{\iota}}^{J}$ is the analytic subgroup of $G$ associated to the subalgebra $\mathfrak{m}_{\lambda_{\iota}}^{J}$. By Corollary 4.41 ,

$$
\left(M_{\lambda_{\iota}}^{J} M_{J}\right) /\left(M_{\lambda_{\iota}}^{J} M_{J}\right) \cap L \cong M_{I} / M_{I} \cap w_{0} H w_{0}^{-1} .
$$

By (4.27) and (3.7) a left invariant integral on $M_{I} / M_{I} \cap w_{0} H w_{0}^{-1}$ is given by

$$
f \mapsto \int_{K \cap M_{I}} d k \int_{a \in \mathfrak{a}^{I,+}} \sum_{w \in \mathcal{W}_{I}} f\left(k a w\left(M_{I} \cap w_{0} H w_{0}^{-1}\right)\right) \delta_{I}(a) d a,
$$

where $f \in C_{c}\left(M_{I} / M_{I} \cap w_{0} H w_{0}^{-1}\right)$, and $d k$ denotes a Haar integral on $K \cap M_{I}$.

Combining (5.6), (5.7), (5.8), and (5.9) we obtain that for all $f \in C_{c}\left(P_{J}\right)$,

$$
f \mapsto \int_{K \cap P} d k \int_{a \in \mathfrak{a}^{I,+}} d a \int_{\bar{b} \in \mathfrak{a}_{J} / \mathfrak{a}_{J} \cap \operatorname{ker} \lambda_{\iota}} d \bar{b} \sum_{w \in \mathcal{W}_{I}} \int_{L} f(k \exp (b) \exp (a) w l) \delta_{I}(a) \exp (2 \rho(b)) d l,
$$

defines a right Haar integral, say $d p$, on $P_{J}$, were $d k$ and $d l$ denote Haar integrals on $K \cap P_{J}$ and $L$, respectively.

Note that a Haar integral on $G$ is given by

$$
f \mapsto \int_{K} d k \int_{P_{J}} f(k p) d p, \quad f \in C_{c}(G),
$$

where $d k$ denotes a Haar integral on $K$. Combining (5.10) and (5.11), and the fact that $L$ is the stabilizer of $\left(v_{0} w_{0}\right)^{I}$ in $G$, we obtain that the formula (5.3) indeed gives a $G$-invariant measure on $G\left(w_{0} v_{0}\right)^{I}$.

It turns out that the sets $I_{\iota}(I)$ satisfy the condition of the above theorem; see (1.16) for the definition. To show this we need the following:

Proposition 5.12. If $I \subset \Delta_{\sigma}$ is $\lambda_{\iota}$-connected, then $I_{\iota}(I)$ is $\lambda_{\iota}$-connected. In fact, any $J \subset \Delta_{\sigma}$ containing $I_{\iota}(I)$ is $\lambda_{\iota}$-connected. 
Proof. Let $I_{0} \supset I$ be the largest $\lambda_{\iota}$-connected subset of $J$, and let $S=J \backslash I_{0}$. Then $S \subset\left(I_{0} \cup\left\{\lambda_{\iota}\right\}\right)^{\perp}$. Therefore $\mathfrak{a}^{S} \subset \mathfrak{a}_{I_{0}} \cap \operatorname{ker} \lambda_{\iota}$. Note that $\mathfrak{a}_{I_{0}}=\mathfrak{a}_{J} \oplus \mathfrak{a}_{\Delta_{\sigma} \backslash S}$. Now given $a \in \mathfrak{a}^{S,+}$, we write $a=x+y$ with $x \in \mathfrak{a}_{\Delta_{\sigma} \backslash S}$ and $y \in \mathfrak{a}_{J}$. Then for any $\alpha \in S \subset J$ we have $\alpha(a) \geq 0$ by (4.9), and $\alpha(y)=0$, and hence $\alpha(x) \geq 0$. And for any $\beta \in \Delta_{\sigma} \backslash S$, we have $\beta(x)=0$. Therefore $x \in \mathfrak{a}^{+}$; in other words,

$$
\mathfrak{a}^{S,+} \subset\left(\mathfrak{a}_{\Delta_{\sigma} \backslash S} \cap \mathfrak{a}^{+}\right)+\mathfrak{a}_{J} .
$$

Therefore, since $I \subset I_{0} \subset \Delta_{\sigma} \backslash S$ and $I_{\iota}(I) \subset J$, we get

$$
\mathfrak{a}^{S,+} \subset\left(\mathfrak{a}_{I} \cap \mathfrak{a}^{+}\right)+\mathfrak{a}_{I_{\iota}(I)} .
$$

By the definition of $I_{\iota}(I)$ as in (1.16), exists $C=a_{\iota}(I)>0$ such that

$$
\begin{aligned}
& \rho(x) \leq C \lambda_{\iota}(x), \quad \forall x \in \mathfrak{a}_{I} \cap \mathfrak{a}^{+}, \quad \text { and } \\
& \rho(y)=C \lambda_{\iota}(y), \quad \forall y \in \mathfrak{a}_{I_{\iota}(I)} .
\end{aligned}
$$

Combining (5.13), (5.14), (5.15), and since $\mathfrak{a}^{S} \subset$ ker $\lambda_{\iota}$, we conclude that

$$
\rho(a) \leq C \lambda_{\iota}(a)=0 \quad \forall a \in \mathfrak{a}^{S,+} .
$$

Since $S \perp I_{0}$, we have $\Sigma_{J}^{+}=\Sigma_{I_{0}}^{+} \cup \Sigma_{S}^{+}$(cf. Definition 4.33). Therefore given $a \in$ $\mathfrak{a}^{S,+} \subset \mathfrak{a}_{I_{0}}$, we have

$$
2 \rho(a)=\operatorname{tr}\left(\left.\operatorname{ad}(a)\right|_{\mathfrak{n}_{J}}\right)+\sum_{\alpha \in \Sigma_{S}^{+}} \operatorname{tr}\left(\left.\operatorname{ad}(a)\right|_{\mathfrak{g}_{\alpha}}\right) .
$$

Recall that $\mathfrak{a}^{S} \subset \mathfrak{m}_{S} \subset \mathfrak{m}_{J}, \mathfrak{m}_{J}$ is semisimple, and $\left[\mathfrak{m}_{J}, \mathfrak{n}_{J}\right] \subset \mathfrak{n}_{J}$. Therefore

$$
\operatorname{tr}\left(\left.\operatorname{ad}(a)\right|_{\mathfrak{n}_{J}}\right)=0 \text {. }
$$

Note that

$$
\operatorname{tr}\left(\left.\operatorname{ad}(a)\right|_{\mathfrak{g}^{\alpha}}\right)=\left(\operatorname{dim} \mathfrak{g}_{\alpha}\right) \alpha(a) \geq 0, \quad \forall \alpha \in \Sigma_{S}^{+} .
$$

Now by (5.17), we get $\rho(a) \geq 0$. Therefore (5.16), we get $\rho(a)=0$. Hence by (5.18),

$$
\left(\operatorname{dim} \mathfrak{g}_{\alpha}\right) \alpha(a)=0, \quad \forall \alpha \in S, \forall a \in \mathfrak{a}^{S,+} .
$$

Now if $S \neq \emptyset$, then for any $\alpha \in S$ : we have $\operatorname{dim} \mathfrak{g}_{\alpha} \geq 1$; and since $\mathfrak{a}^{S} \perp \mathfrak{a}_{S}$, we have $\alpha(a) \neq 0$ for any $0 \neq a \in \mathfrak{a}^{S}$. This contradicts (5.19). Hence $S=\emptyset$; that is, $J$ is $\lambda_{\iota}$-connected.

Corollary 5.20. Let $I \subset \Delta_{\sigma}$ be $\lambda_{\iota}$-connected. Then for any $w_{0} \in \mathcal{W}$, the orbit $G\left(w_{0} v_{0}\right)^{I_{\iota}(I)}$ admits a $G$-invariant measure, say $\nu_{I_{\iota}(I), w_{0}}$, such that for any $f \in$ $C_{c}\left(G\left(w_{0} v_{0}\right)^{I_{L}(I)}\right)$,

$$
\int_{W} f d \nu_{I_{\iota}(I), w_{0}}=\int_{K} d k \int_{\bar{a} \in \mathfrak{c}^{+}} \sum_{w \in \mathcal{W}_{I_{\iota}(I)}} f\left(k \exp (a) w\left(w_{0} v_{0}\right)^{I_{\iota}(I)}\right) \xi_{I_{\iota}(I)}(a) d \bar{a},
$$

where

$$
\begin{aligned}
\xi_{I_{\iota}(I)}(a) & :=\delta_{I_{\iota}(I)}(a) \exp \left(\operatorname{tr}\left(\left.\operatorname{ad} a\right|_{\mathfrak{n}_{I_{\iota}(I)}}\right)\right), \\
\mathfrak{e}^{+} & =\left\{\bar{a} \in \mathfrak{a} / \mathfrak{a}_{I_{\iota}(I)} \cap \operatorname{ker} \lambda_{\iota}: \alpha(a) \geq 0, \forall \alpha \in I_{\iota}(I)\right\},
\end{aligned}
$$


and $d \bar{a}$ denotes the Lebesgue integral on $\mathfrak{e}^{+}$.

Proof. By Proposition $5.12 J\left(I_{\iota}(I)\right)=I_{\iota}(I)$. By (5.15) for any $y \in \mathfrak{a}_{I_{\iota}(I)}, \rho(y)=0$ if and only if $\lambda_{\iota}(y)=0$. Therefore by Theorem 5.1, $G\left(w_{0} v_{0}\right)^{I}$ admits a $G$-invariant measure.

Put $\mathfrak{E}=\mathfrak{a}_{I_{\iota}(I)} \cap \operatorname{ker} \lambda_{\iota}$. Then the map $\mathfrak{a}^{I_{\iota}(I)} \oplus \mathfrak{a}_{I_{\iota}(I)} / \mathfrak{E} \rightarrow \mathfrak{a} / \mathfrak{E}$, given by

$$
(a, b+\mathfrak{E}) \mapsto(a+b)+\mathfrak{E}, \quad \forall a \in \mathfrak{a}^{I_{L}(I)}, \forall b \in \mathfrak{a}_{I_{\iota}(I)},
$$

is an isomorphism. Note that $\delta_{I_{\iota}(I)}(b)=1$ and $\operatorname{tr}\left(\left.\operatorname{ad} a\right|_{\mathfrak{n}_{I_{\ell}(I)}}\right)=0$. Therefore $\xi_{I_{\iota}(I)}$ is well defined on $\mathfrak{a} / \mathfrak{E}$. Moreover $(a+b)+\mathfrak{E} \in \mathfrak{e}^{+}$if and only if $a \in \mathfrak{a}^{I_{\iota}(I),+}$. Therefore (5.21) follows from (5.3).

\section{Volume Asymptotics}

In this section we derive some formulas for the volume asymptotics (see also GW] and $[\mathrm{M}]$ for a similar computation).

6.1. Basic asymptotic formula. Consider a space $\mathfrak{a} \simeq \mathbb{R}^{r}$ and a map

$$
\phi: \mathfrak{a} \rightarrow W: a \mapsto \sum_{i=1}^{k} e^{\lambda_{i}(a)} w_{i} .
$$

where $W$ is a finite-dimensional vector space, $w_{1}, \ldots, w_{k} \in W$ are linearly independent vectors, and $\lambda_{1}, \ldots, \lambda_{k}$ are (additive) characters.

Fix a basis $\Delta$ of the dual space $\mathfrak{a}^{*}$ and set

$$
\mathfrak{a}^{+}=\{a \in \mathfrak{a}: \alpha(a) \geq 0 \quad \text { for } \alpha \in \Delta\} .
$$

We assume that

(1) $\lambda_{1}=\sum_{\alpha \in \Delta} m_{\alpha} \alpha$ with $m_{\alpha}>0$.

(2) $\lambda_{i} \leq \lambda_{1}$ for all $i$, that is, $\lambda_{1}-\lambda_{i} \in \sum_{\alpha \in \Delta} m_{i, \alpha} \alpha$. with $m_{i, \alpha} \geq 0$.

Let

$$
\operatorname{supp}\left(\lambda_{i}\right)=\left\{\alpha \in \Delta: m_{i, \alpha}>0\right\}
$$

For

$$
\chi=\sum_{\alpha \in \Delta} v_{\alpha}(\chi) \alpha \in \mathfrak{a}^{*}
$$

we set

$$
a_{\chi}:=\max \left\{\frac{v_{\alpha}(\chi)}{m_{\alpha}}: \alpha \in \Delta\right\}, I_{\chi}:=\left\{\alpha \in \Delta: \frac{v_{\alpha}(\chi)}{m_{\alpha}}<a_{\chi}\right\}, b_{\chi}:=\#\left(\Delta-I_{\chi}\right) .
$$

Define $\operatorname{ker} I_{\chi}:=\cap_{\alpha \in I_{\chi}} \operatorname{ker} \alpha$. Then

$$
\begin{gathered}
\chi(a)=a_{\chi} \cdot \lambda_{1}(a), \quad \forall a \in \operatorname{ker} I_{\chi}, \\
\lambda_{i}(a)=\lambda_{1}(a), \quad \forall i: \operatorname{supp} \lambda_{i} \subset I_{\chi}, \quad \forall a \in \operatorname{ker} I_{\chi}, \\
\mathfrak{d}_{0}:=\operatorname{ker} \chi \cap \operatorname{ker} I_{\chi}=\operatorname{ker} \lambda_{i} \cap \operatorname{ker} I_{\chi}, \quad \forall i: \operatorname{supp} \lambda_{i} \subset I_{\chi} .
\end{gathered}
$$


Therefore we can define

$$
\begin{aligned}
\mathfrak{d}^{+} & :=\left\{\bar{a} \in \mathfrak{a} / \mathfrak{d}_{0}: \alpha(a) \geq 0, \forall \alpha \in I_{\chi}\right\}, \\
\psi(\bar{a}) & :=\sum_{i: \operatorname{supp} \lambda_{i} \subseteq I_{\chi}} e^{\lambda_{i}(a)} w_{i}, \quad \forall \bar{a} \in \mathfrak{a} / \mathfrak{d}_{0}, \quad \text { and } \\
L_{\chi}(f) & :=\int_{\mathfrak{d}^{+}} f(\psi(\bar{a})) e^{\chi(a)} d \bar{a}, \quad \forall f \in C_{c}(W),
\end{aligned}
$$

where $d \bar{a}$ denotes the Lebesgue measure on $\mathfrak{a} / \mathfrak{d}_{0}$.

The main result of this subsection is the following theorem

Theorem 6.1. For $\chi \in \mathfrak{a}^{*}$ and $f \in C_{c}(W)$,

$$
\lim _{T \rightarrow \infty} \frac{1}{T^{a_{\chi}(\log T)^{b_{\chi}-1}}} \int_{\mathfrak{a}^{+}} f(\phi(a) / T) e^{\chi(a)} d a=\kappa_{\chi} \cdot L_{\chi}(f)<\infty,
$$

where

$$
\kappa_{\chi}=\operatorname{Vol}\left(\mathfrak{a}^{+} \cap \operatorname{ker}\left(I_{\chi}\right) \cap\left\{\lambda_{1}=1\right\}\right) .
$$

We start the proof with a lemma:

Lemma 6.2. $\quad$ (a) For $T>0$, let $\mathfrak{a}_{T}^{+}:=\left\{a \in \mathfrak{a}^{+}: e^{\lambda_{1}(a)} \leq T\right\}$. Then

$$
\int_{\mathfrak{a}_{T}^{+}} e^{\chi(a)} d a \ll T^{a_{\chi}}(\log T)^{b_{\chi}-1} .
$$

(b) For $\lambda_{i}$ such that $\operatorname{supp} \lambda_{i} \nsubseteq I_{\chi}$ and $T, \delta>0$, set

$$
\mathfrak{a}_{T}^{+}(i, \delta)=\left\{a \in \mathfrak{a}_{T}^{+}: e^{\lambda_{i}(a)} \geq \delta T\right\} .
$$

Then for some constant $C_{\delta}>1$ depending on $\delta$,

$$
\int_{\mathfrak{a}_{T}^{+}(i, \delta)} e^{\chi(a)} d a \leq C_{\delta} T^{a_{\chi}}(\log T)^{b_{\chi}-2} .
$$

Proof. To prove (a), we use induction on $\left|I_{\chi}\right|$. If $I_{\chi}=\emptyset$, then $\chi=a_{\chi} \cdot \lambda_{1}$ and

$$
\begin{aligned}
\int_{a \in \mathfrak{a}^{+}: \lambda_{1}(a) \leq \tau} e^{\chi(a)} d a & =\int_{0}^{\tau} \operatorname{Vol}\left(\mathfrak{a}^{+} \cap\left\{\lambda_{1}=s\right\}\right) e^{a_{\chi} s} d s \\
& =\int_{0}^{\tau}\left(c s^{r-1}\right) e^{a_{\chi} s} d s=O\left(\tau^{r-1} e^{a_{\chi} \tau}\right) .
\end{aligned}
$$

Let $\alpha \in I_{\chi}$ and $\mathfrak{b}^{+}=\mathfrak{a}^{+} \cap \operatorname{ker}(\alpha)$. Then by the inductive assumption,

$$
\begin{aligned}
& \int_{a \in \mathfrak{a}^{+}: \lambda_{1}(a) \leq \tau} e^{\chi(a)} d a=\int_{0}^{\tau / m_{\alpha}} e^{v_{\alpha}(\chi) s}\left(\int_{b \in \mathfrak{b}^{+}: \lambda_{1}(b) \leq \tau-m_{\alpha} s} e^{\chi(b)} d b\right) d s \\
\ll & \int_{0}^{\tau / m_{\alpha}} e^{v_{\alpha}(\chi) s}\left(\tau-m_{\alpha} s\right)^{b_{\chi}-1} e^{a_{\chi}\left(\tau-m_{\alpha} s\right)} d s \ll e^{a_{\chi} \tau} \tau^{b_{\chi}-1},
\end{aligned}
$$

where $C>1$ is a constant. This proves (a). 
To prove (b), we write $\lambda_{i}=\lambda_{1}-\sum_{\alpha \in \operatorname{supp}\left(\lambda_{i}\right)} m_{i, \alpha} \alpha$ with $m_{i, \alpha}>0$. For $a \in \mathfrak{a}_{T}^{+}(i, \delta)$, we have

$$
\sum_{\alpha \in \operatorname{supp} \lambda_{i}} m_{i, \alpha} \alpha(a) \leq-\log \delta .
$$

Setting $\mathfrak{c}=\operatorname{ker}\left(\operatorname{supp} \lambda_{i}\right)$ and $\mathfrak{c}_{T}^{+}=\mathfrak{a}_{T}^{+} \cap \mathfrak{c}$, we get

$$
\int_{\mathfrak{a}_{T}^{+}(i, \delta)} e^{\chi(a)} d a \leq C_{\delta} \int_{\mathfrak{c}_{T}^{+}} e^{\chi(c)} d c
$$

for some constant $C_{\delta}>1$ depending on $\delta$. Since $\operatorname{supp} \lambda_{i} \nsubseteq I_{\chi}, b_{\left.\chi\right|_{\mathfrak{c}}} \leq b_{\chi}-1$, and (b) follows from (a).

Proof of Theorem 6.1. There exists $c=c(f)>0$ such that if $f(\phi(a) / T) \neq 0$ or $f(\psi(a) / T) \neq 0$, then $a \in \mathfrak{a}_{c T}^{+}$.

For $\delta>0$, set

$$
\mathfrak{a}_{T}^{+}(\delta)=\bigcup_{i: \operatorname{supp}\left(\lambda_{i}\right) \nsubseteq I_{\chi}} \mathfrak{a}_{T}^{+}(i, \delta) .
$$

where $\mathfrak{a}_{T}^{+}(i, \delta)$ is defined as in Lemma 6.2. By uniform continuity, for every $\varepsilon>0$, there exists $\delta>0$ such that for $a \in \mathfrak{a}_{c T}^{+}-\mathfrak{a}_{c T}^{+}(\delta)$,

$$
|f(\phi(a) / T)-f(\psi(a) / T)|<\varepsilon .
$$

Hence, by Lemma 6.2,

$$
\begin{aligned}
& \left|\int_{\mathfrak{a}^{+}} f(\phi(a) / T) e^{\chi(a)} d a-\int_{\mathfrak{a}^{+}} f(\psi(a) / T) e^{\chi(a)} d a\right| \\
\leq & \int_{\mathfrak{a}_{c T}^{+}-\mathfrak{a}_{c T}^{+}(\delta)} \varepsilon \cdot e^{\chi(a)} d a+\int_{\mathfrak{a}_{c T}^{+}(\delta)}\left(2\|f\|_{\infty}\right) \cdot e^{\chi(a)} d a \\
= & O_{f}\left(\varepsilon \cdot T^{a_{\chi}}(\log T)^{b_{\chi}-1}\right)+O_{f, \delta}\left(T^{a_{\chi}}(\log T)^{b_{\chi}-2}\right) .
\end{aligned}
$$

This shows that

$$
\int_{\mathfrak{a}^{+}} f(\phi(a) / T) e^{\chi(a)} d a=\int_{\mathfrak{a}^{+}} f(\psi(a) / T) e^{\chi(a)} d a+o\left(T^{a_{\chi}}(\log T)^{b_{\chi}-1}\right) .
$$

Let

$$
\mathfrak{s}^{+}=\mathfrak{a}^{+} \cap \operatorname{ker}\left(I_{\chi}\right) \quad \text { and } \quad \mathfrak{t}^{+}=\mathfrak{a}^{+} \cap \operatorname{ker}\left(\Delta-I_{\chi}\right) .
$$

There exists $c=c(f)>0$ such that if $f\left(\psi(t) e^{u}\right) \neq 0$ for some $t \in \mathfrak{t}^{+}$and $u \in \mathbb{R}$, then $e^{\lambda_{1}(t)} \leq c e^{-u}$ and $e^{u} \leq c$. Using that for some $\varepsilon>0$,

$$
\left.\chi\right|_{\mathfrak{t}^{+}} \leq\left.\left(a_{\chi}-\varepsilon\right) \cdot \lambda_{1}\right|_{\mathfrak{t}^{+}},
$$


we deduce that

$$
\begin{aligned}
\int_{\mathfrak{t}^{+}} \int_{-\infty}^{\infty} & f\left(\psi(t) e^{u}\right) e^{\chi(t)+a_{\chi} u}|u|^{l} d u d t \\
& \ll \int_{u=-\infty}^{\log c} d u \int_{\left\{t \in \mathfrak{t}^{+}: \lambda_{1}(t) \leq \log c-u\right\}} e^{\left(a_{\chi}-\varepsilon\right) \lambda_{1}(t)+a_{\chi} u}|u|^{l} d t \\
& \ll \int_{-\infty}^{\log c} e^{\varepsilon u}(\log c-u)^{r-b_{\chi}}|u|^{l} d u<\infty
\end{aligned}
$$

for every $l \in \mathbb{N} \cup\{0\}$. In particular, putting $l=0$ we get

$$
L_{\chi}(f)=\int_{\mathfrak{d}^{+}} f(\psi(\bar{a})) e^{\chi(a)} d \bar{a}=\int_{t \in \mathfrak{t}^{+}} \int_{u=-\infty}^{\infty} f\left(\psi(t) e^{u}\right) e^{\chi(t)+a_{\chi} u} d t d u<\infty .
$$

Therefore applying (6.3), we conclude that as $T \rightarrow \infty$,

$$
\begin{aligned}
\int_{\mathfrak{a}^{+}} f(\psi(a) / T) e^{\chi(a)} d a & =\int_{\mathfrak{t}^{+}} \int_{\mathfrak{s}^{+}} f\left(\psi(t) e^{\lambda_{1}(s)} / T\right) e^{\chi(t)+a_{\chi} \lambda_{1}(s)} d s d t \\
& =\int_{\mathfrak{t}^{+}} \int_{0}^{\infty} f\left(\psi(t) e^{u} / T\right) e^{\chi(t)+a_{\chi} u} \cdot \operatorname{Vol}\left(\mathfrak{s}^{+} \cap\left\{\lambda_{1}=u\right\}\right) d u d t \\
& =\int_{\mathfrak{t}^{+}} \int_{-\log T}^{\infty} f\left(\psi(t) e^{u}\right) e^{\chi(t)+a_{\chi} u} T^{a_{\chi}} \cdot \kappa_{\chi}(u+\log T)^{b_{\chi}-1} d u d t \\
& =\kappa_{\chi} \cdot L_{\chi}(f) \cdot T^{a_{\chi}}(\log T)^{b_{\chi}-1}+o\left(T^{a_{\chi}} \cdot(\log T)^{b_{\chi}-1}\right) .
\end{aligned}
$$

This completes the proof.

6.2. Volume of symmetric space. Let $G$ be a connected noncompact semisimple Lie group with finite center, $H$ its symmetric subgroup, and $\iota: G \rightarrow \mathrm{GL}(W)$ be an almost faithful irreducible over $\mathbb{R}$ representation. We assume that $H=\operatorname{Stab}_{G}\left(v_{0}\right)$ for some $v_{0} \in W$. We use notation from Section 3. In particular, $\mu$ denotes an invariant measure on $G / H$ and

$$
\lambda_{\iota}=\sum_{\alpha \in \Delta_{\sigma}} m_{\alpha} \alpha \in \mathfrak{a}^{*}, \quad m_{\alpha} \in \mathbb{Q}^{+},
$$

is the highest weight of $\iota$. Let $a_{\iota}, b_{\iota}, I_{\iota}$ be defined as in (1.10).

Theorem 6.4. For every $f \in C_{c}(W)$,

$$
\lim _{T \rightarrow \infty} \frac{1}{T^{a_{\iota}}(\log T)^{b_{\iota}-1}} \int_{G / H} f\left(g v_{0} / T\right) d \mu(g)=\int_{W} f d \nu_{\iota},
$$

where $\nu_{\iota}$ is a locally finite $G$-invariant measure on $W$ concentrated on $\mathbb{R}^{+} \cdot V_{I_{\iota}}^{\infty}$.

Moreover when considered as a measure on $\mathbb{R}^{+} \cdot V_{I_{\iota}}^{\infty}, \nu_{\iota}$ is a linear combination of measures $\nu_{I_{\iota}, w}, w \in \mathcal{W}^{I_{\iota}}$, given in (5.21).

Proof. For $v \in W$, set

$$
\Phi_{\iota}(v)=\left\{\lambda \in \Phi_{\iota}: v^{\lambda} \neq 0\right\}
$$


By (3.7),

$$
\begin{aligned}
\int_{G / H} f\left(g v_{0} / T\right) d \mu(g) & =\int_{K} \sum_{w \in \mathcal{W}} \int_{\mathfrak{a}^{+}} f\left(k \exp (a) w v_{0} / T\right) \xi(a) d a d k \\
& =\int_{K} \sum_{w \in \mathcal{W}} \int_{\mathfrak{a}^{+}} f\left(k \cdot \sum_{\lambda \in \Phi_{\iota}\left(w v_{0}\right)} e^{\lambda(a)}\left(w v_{0}\right)^{\lambda} / T\right) \xi(a) d a d k
\end{aligned}
$$

where

$$
\xi(a)=\prod_{\alpha \in \Sigma^{+}}(\sinh \alpha(a))^{l_{\alpha}^{+}}(\cosh \alpha(a))^{l_{\alpha}^{-}}=\sum_{\chi \in \Xi} t_{\chi} e^{\chi(a)}
$$

for some $t_{\chi} \neq 0$ and $\Xi \subset \mathfrak{a}^{*}$. Let $a_{\iota}, b_{\iota}$, and $I_{\iota}$ be as defined in (1.10). Let

$$
\Xi^{\prime}=\left\{\chi \in \Xi: a_{\chi}=a_{\iota}, b_{\chi}=b_{\iota}\right\} \quad \text { and } \quad \Xi^{\prime \prime}=\Xi-\Xi^{\prime} \text {. }
$$

Note that for $\chi \in \Xi^{\prime \prime}$, we have $a_{\chi} \leq a_{\iota}=a_{2 \rho}$ and if $a_{\chi}=a_{\iota}$, then $b_{\chi}<b_{\iota}=b_{2 \rho}$ and $I_{\chi} \supset I_{\iota}=I_{2 \rho}$.

Since by Lemma 4.2, $\lambda_{\iota} \in \Phi_{\iota}\left(w v_{0}\right)$, the assumptions of Section 6.1 are satisfied and applying Theorem 6.1 together with the dominated convergence theorem, we deduce that (6.5) holds with the measure $\nu_{\iota}$ given by the formula

$$
\int_{W} f d \nu_{\iota}=\kappa_{2} \rho \int_{K} \sum_{w \in \mathcal{W}} \int_{\mathfrak{d}^{+}} f\left(k \exp (a)\left(w v_{0}\right)^{I_{\iota}}\right) \xi_{I_{\iota}}(a) d a d k
$$

where

$$
\begin{aligned}
\mathfrak{d}^{+} & =\left\{a \in \mathfrak{a} /\left(\operatorname{ker}\left(I_{\iota}\right) \cap \operatorname{ker}(\rho)\right): \alpha(a) \geq 0, \alpha \in I_{\iota}\right\}, \\
\xi_{I_{\iota}}(a) & =\sum_{\chi \in \Xi^{\prime}} t_{\chi} e^{\chi(a)} .
\end{aligned}
$$

Note that by Theorem 6.1 the limit in (6.5) is finite (i.e., $\nu_{\iota}$ is locally finite). Also, it is clear from (6.5) that $\nu_{\iota}$ is $G$-invariant and homogeneous of degree $a_{\iota}$. It follows from Proposition 4.35 that

$$
G\left(w v_{0}\right)^{I_{\iota}}=K \exp \left(\mathfrak{d}^{+}\right) \mathcal{W}_{I_{\iota}}\left(w v_{0}\right)^{I_{\iota}}=\mathbb{R}^{+} \cdot K \exp \left(\mathfrak{a}^{I_{\iota},+}\right) \mathcal{W}_{I_{\iota}}\left(w v_{0}\right)^{I_{\iota}} .
$$

Note that

$$
V_{I_{\iota}}^{\infty}=\cup_{w \in \mathcal{W}} \pi\left(G\left(w v_{0}\right)^{I_{\iota}}\right)=\cup_{w \in \mathcal{W}^{I_{\iota}}} \pi\left(G\left(w v_{0}\right)^{I_{\iota}}\right) .
$$

Since for $\chi \in \Xi$, we have

$$
\chi \in \Xi^{\prime} \quad \text { if and only if } \quad \chi \in 2 \rho+\left\langle I_{\iota}\right\rangle,
$$

it follows that the formula for (6.9) for $\xi_{I_{\iota}}$ is same as the formula (5.22) of Corollary 5.20 for $I_{\iota}=I_{\iota}(\emptyset)$. Note that each $G$-orbit $G\left(w v_{0}\right)^{I_{\iota}}$ is a closed subset of $\mathbb{R}^{+} \cdot V_{I_{\iota}}^{\infty}$ and hence $f \in C_{c}\left(\mathbb{R}^{+} \cdot V_{I_{\iota}}^{\infty}\right)$ implies the restriction of $f$ to $G\left(w v_{0}\right)^{I_{\iota}}$ belongs to $C_{c}\left(G\left(w v_{0}\right)^{I_{\iota}}\right)$. Hence (6.8) is in agreement with (5.21) for $f \in C_{c}\left(\mathbb{R}^{+} \cdot V_{I_{\iota}}^{\infty}\right)$.

This shows that $\nu_{\iota}$, considered as a measure on $\mathbb{R}^{+} \cdot V_{I_{\iota}}^{\infty}$, is a linear combination of the measures $\nu_{I_{\iota}, w}, w \in \mathcal{W}^{I_{\iota}}$, given in (5.21). It follows that $\nu_{\iota}$ is concentrated on $\mathbb{R}^{+} \cdot V_{I_{\iota}}^{\infty}$. 
We can easily deduce the following volume asymptotic of balls from the above theorem:

Corollary 6.10. For any norm $\|\cdot\|$ on $W$,

$$
\operatorname{Vol}(\{v \in V:\|v\|<T\}) \sim c \cdot T^{a_{\iota}}(\log T)^{b_{\iota}} \quad \text { as } T \rightarrow \infty
$$

where Vol denotes a $G$-invariant measure on $V$ and $c>0$.

Remark 6.11. Theorem 6.4 holds for a representation $\iota$ which is not irreducible. Let

$$
\begin{aligned}
& \mathcal{P}=\left\{a \in \mathfrak{a}^{+}: \lambda(a) \leq 1, \lambda \in \Phi_{\iota}\right\}, \\
& a_{\iota}=\max \{2 \rho(a): a \in \mathcal{P}\}, \\
& b_{\iota}=\operatorname{dim} \mathcal{P} \cap\left\{2 \rho=a_{\iota}\right\} .
\end{aligned}
$$

Then for every $f \in C_{c}(W)$,

$$
\lim _{T \rightarrow \infty} \frac{1}{T^{a_{\iota}}(\log T)^{b_{\iota}-1}} \int_{G / H} f\left(g v_{0} / T\right) d \mu(g)=\int_{W} f d \nu_{\iota},
$$

where $\nu_{\iota}$ is a $G$-invariant measure concentrated on a union of finitely many $G$-orbits. To adapt the proof to this case, we decompose the polyhedron $\mathcal{P}$ into a finite union of symplicial polyhedra $\mathcal{P}_{i}$. the asymptotics for the integral over $K \exp \left(\mathcal{P}_{i}\right) \mathcal{W} H$ can be computed from Theorem 6.1. Using the argument from Section 7, we also get the asymptotics for integral points.

For $f \in C_{c}(W \backslash\{0\})$ with $\pi(\operatorname{supp} f) \cap V^{\infty} \neq \emptyset$, we define $a_{\iota}(f), b_{\iota}(f), \Theta_{\iota}(f)$ as in (1.17) with $\Omega=\pi(\operatorname{supp} f)$. Similarly, we define $\Theta_{f}$ and $\Theta_{f}^{\min }$.

Theorem 6.12. For every $f \in C_{c}(W \backslash\{0\})$ with $\pi(\operatorname{supp} f) \cap V^{\infty} \neq \emptyset$,

$$
\lim _{T \rightarrow \infty} \frac{1}{T^{a_{\iota}(f)}(\log T)^{b_{\iota}(f)-1}} \int_{G / H} f\left(g v_{0} / T\right) d \mu(g)=\int_{W} f d \nu_{\Theta_{\iota}(f)},
$$

where $\nu_{\Theta_{\iota}(f)}$ is a G-invariant measure on $W$ which is concentrated on and locally finite on

$$
\cup_{I \in \Theta_{\iota}(f)} \mathbb{R}^{+} \cdot V_{I}^{\infty}
$$

In particular, $\nu_{\Theta_{\iota}(f)}(\operatorname{supp} f)<\infty$.

Proof. Define $\tilde{W}=\mathbb{R}^{+} \cdot \overline{\pi(V)}$ and $S(\tilde{W}):=S(W) \cap \tilde{W}=\pi(\tilde{W})$, where $S(W)$ and $\pi: W \rightarrow S(W)$ are defined as in (1.5). Since $f \in C_{c}(W \backslash\{0\}),\left.f\right|_{\tilde{W}} \in C_{c}(\tilde{W})$. Hence it suffices to prove the theorem for $f \in C_{c}(\tilde{W})$. For $I \subset \Delta_{\sigma}$, we set

$$
\mathcal{O}_{I}=\bigcup_{\lambda_{\iota} \text {-connected } J \supset I} V_{J}^{\infty} \text {. }
$$

It follows from Proposition 4.14 that $\mathcal{O}_{I}$ is open in $S(\tilde{W})$. We take a partition of unity $\phi_{I} \in C(S(\tilde{W})), I \in \Theta_{f}^{\min }$, associated to the cover

$$
\pi(\operatorname{supp} f) \subset \bigcup_{I \in \Theta_{f}^{\min }} \mathcal{O}_{I} .
$$


It suffices to prove the theorem for the functions $f_{I}(v)=f(v) \phi_{I}(\pi(v)), I \in \Theta_{f}^{\min }$. Hence, we may assume that $\Theta_{f}^{\min }=\{I\}$ for some $\lambda_{\iota}$-connected $I \subset \Delta_{\sigma}$. Then

$$
\left(a_{\iota}(f), b_{\iota}(f)\right)=\left(a_{\iota}(I), b_{\iota}(I)\right) \text { and } \Theta_{\iota}(f)=\left\{I_{\iota}(I)\right\} .
$$

By Corollary 4.42, there exists a compact set $U \subset \mathfrak{a}^{I,+}$ such that

$$
\begin{aligned}
& \pi(\operatorname{supp} f) \cap V^{\infty} \subset \bigcup_{I \in \Theta_{f}} \pi\left(K \exp \left(U+\mathfrak{a}_{I}^{+}\right)\left(\mathcal{W} v_{0}\right)^{I}\right), \\
& \pi(\operatorname{supp} f) \cap \pi(V) \subset \pi\left(K \exp \left(U+\mathfrak{a}_{I}^{+}\right) \mathcal{W} v_{0}\right) .
\end{aligned}
$$

Hence, as in (6.6), we have

$$
\begin{aligned}
& \int_{G / H} f\left(g v_{0} / T\right) d \mu(g) \\
& =\int_{K} \sum_{w \in \mathcal{W}} \int_{U} \int_{\mathfrak{a}_{I}^{+}} f\left(k \cdot \sum_{\lambda \in \Phi_{\iota}\left(w v_{0}\right)} e^{\lambda(a)} e^{\lambda(u)}\left(w v_{0}\right)^{\lambda} / T\right) \xi(a+u) d a d u d k .
\end{aligned}
$$

We apply Theorem 6.1 to the integral over $\mathfrak{a}_{I}^{+}$in place of $\mathfrak{a}$. For $\chi=\sum_{\alpha \in \Delta_{\sigma}} v_{\alpha} \alpha$, we set

$$
\begin{aligned}
& a_{\chi}:=\max \left\{\frac{v_{\alpha}}{m_{\alpha}}: \alpha \in \Delta_{\sigma} \backslash I\right\}, \\
& I_{\chi}:=\left\{\alpha \in \Delta_{\sigma} \backslash I: \frac{v_{\alpha}}{m_{\alpha}}<a_{\chi}\right\}, \\
& b_{\chi}:=\#\left(\left(\Delta_{\sigma} \backslash I\right) \backslash I_{\chi}\right) .
\end{aligned}
$$

We write

$$
\xi(a)=\sum_{\chi \in \Xi} t_{\chi} e^{\chi(a)}
$$

for some $t_{\chi} \neq 0$ and $\Xi \subset \mathfrak{a}^{*}$. Let

$$
\Xi^{\prime}=\left\{\chi \in \Xi: a_{\chi}=a_{2 \rho}, b_{\chi}=b_{2 \rho}\right\} \quad \text { and } \quad \Xi^{\prime \prime}=\Xi-\Xi^{\prime}
$$

Note that for $\chi \in \Xi^{\prime \prime}$, we have $a_{\chi} \leq a_{2 \rho}=a_{\iota}(I)$ and if $a_{\chi}=a_{2 \rho}$, then $b_{\chi}<b_{2 \rho}=b_{\iota}(I)$ and $I_{\chi} \supset I_{2 \rho}$ where $I_{2 \rho} \cup I=I_{\iota}(I)$. By Theorem 6.1 and the dominated convergence theorem,

$$
\begin{aligned}
& \lim _{T \rightarrow \infty} \frac{1}{T^{a_{2 \rho}(\log T)^{b_{2 \rho}-1}}} \int_{G / H} f\left(g v_{0} / T\right) d \mu(g) \\
= & \kappa \int_{K} \sum_{w \in \mathcal{W}} \int_{U} \int_{\mathfrak{d}^{+}} f\left(k \exp (u+a)\left(w v_{0}\right)^{I_{\iota}(I)}\right) \xi_{I_{\iota}(I)}(u+a) d a d u d k \\
= & \kappa \int_{K} \sum_{w \in \mathcal{W}} \int_{\mathfrak{e}^{+}} f\left(k \exp (a)\left(w v_{0}\right)^{I_{\iota}(I)}\right) \xi_{I_{\iota}(I)}(a) d a d k
\end{aligned}
$$


where

$$
\begin{aligned}
\kappa & =\operatorname{Vol}\left(\mathfrak{a}_{I}^{+} \cap \operatorname{ker}\left(I_{2 \rho}\right) \cap\left\{\lambda_{\iota}=1\right\}\right), \\
\mathfrak{d}^{+} & =\left\{a \in \mathfrak{a}_{I} /\left(\operatorname{ker}\left(I_{2 \rho}\right) \cap \operatorname{ker}(\rho)\right): \alpha(a) \geq 0, \alpha \in I_{2 \rho}\right\}, \\
\mathfrak{e}^{+} & =\left\{a \in \mathfrak{a} /\left(\operatorname{ker}\left(I_{\iota}(I)\right) \cap \operatorname{ker}(\rho)\right): \alpha(a) \geq 0, \alpha \in I_{\iota}(I)\right\}, \\
\xi_{I_{\iota}(I)}(a) & =\sum_{\chi \in \Xi^{\prime}} t_{\chi} e^{\chi(a)} .
\end{aligned}
$$

Therefore (6.13) holds with the measure $\nu_{\Theta_{\iota}(f)}$ given by the formula

$$
\int_{W} f d \nu_{\Theta_{\iota(f)}}=\kappa \int_{K} \sum_{w \in \mathcal{W}} \int_{\mathfrak{e}^{+}} f\left(k \exp (a)\left(w v_{0}\right)^{I_{\iota}(I)}\right) \xi_{I_{\iota}(I)}(a) d a d k
$$

It is clear that $\nu_{\Theta_{\iota}(f)}$ is $G$-invariant and homogeneous of degree $a_{\iota}(f)$. Also, it follows from Proposition 4.35 that

$$
K \exp \left(\mathfrak{e}^{+}\right) \mathcal{W}_{I_{\iota}(I)}\left(w v_{0}\right)^{I_{\iota}(I)}=\mathbb{R}^{+} \cdot K \exp \left(\mathfrak{a}^{I_{\iota}(I),+}\right) \mathcal{W}_{I_{\iota}(I)}\left(w v_{0}\right)^{I_{\iota}(I)}
$$

is a single $G$-orbit. It follows from Theorem 6.1 that $\nu_{\Theta_{\iota}(f)}$ is locally finite on $\mathbb{R}^{+} \cdot V_{I_{\iota}(I)}^{\infty}$.

Since $\chi \in \Xi^{\prime}$ if and only if $\chi \in 2 \rho+\Sigma_{I_{\iota}(I)}$, the formula (6.15) for $\xi_{I_{\iota}(J)}$ is same as (5.22) in Corollary 5.20, Hence (5.21) agrees with (6.14). Since $\xi_{I_{\iota}(I)} \neq 0$ on a set of full Lebesgue measure on $\mathfrak{d}^{+}$, the limit measure is strictly positive on nonempty open subsets of $G\left(\mathcal{W} v_{0}\right)^{I_{\iota}(I)}$. This shows that $\nu_{\Theta_{\iota}(f)}$ is concentrated on $\mathbb{R}^{+} \cdot V_{I_{\iota}(I)}^{\infty}$, proving the theorem.

Remark 6.16. For any $f \in C_{c}(W \backslash\{0\})$ and a $\lambda_{\iota}$-connected $I \subset \Delta_{\sigma}$ satisfying the conditions of Theorem 5.1, if

$$
\left(a_{\iota}(f), b_{\iota}(f)\right)<\left(a_{\iota}(I), b_{\iota}(I)\right) \leq(a, b),
$$

with respect to the lexicographic order on the pairs, then by Theorem 6.12,

$$
\lim _{T \rightarrow \infty} \frac{1}{T^{a}(\log T)^{b-1}} \int_{G / H} f\left(g v_{0} / T\right) d g=0=\int_{W} f d \nu_{I},
$$

where $\nu_{I}$ is a $G$-invariant measure on $W$ concentrated on $\mathbb{R}^{+} \cdot V_{I}^{\infty}$.

\section{Distribution of integral POINTS}

Let $G$ be a connected noncompact semisimple Lie group with finite center, $H$ a symmetric subgroup $G$, and $\iota: G \rightarrow \mathrm{GL}(W)$ an almost faithful irreducible over $\mathbb{R}$ representation of $G$ such that for some $v_{0} \in W, \operatorname{Stab}_{G}\left(v_{0}\right)=H$.

Let $\Gamma$ be an irreducible lattice in $G$ such that $H \cap \Gamma$ is a lattice in $H$. We choose Haar measures $d g d h, d \mu$ on $G, H, G / H$ respectively such that

$$
\int_{G} f d g=\int_{G / H} \int_{H} f(g h) d h d \mu(g), \quad f \in C_{c}(G) .
$$

It is convenient to normalize the measures so that

$$
\operatorname{Vol}(G / \Gamma)=\operatorname{Vol}(H /(H \cap \Gamma))=1 .
$$

The following result was proved in [EM] (see also [DRS]): 
Theorem 7.1. For every $\phi \in C_{c}(G / \Gamma)$,

$$
\int_{H /(H \cap \Gamma)} \phi(v h) d h \rightarrow \int_{G / \Gamma} \phi d g \quad \text { as } v \rightarrow \infty \text { in } G / H .
$$

Remark 7.2. The condition that the lattice $\Gamma$ is irreducible in $G$ can be relaxed. In fact, it suffices to assume that $G=G_{1} \cdots G_{r}$ for noncompact normal subgroups $G_{i}$ 's such that $\Gamma \cap G_{i}$ is an irreducible lattice in $G_{i}$ and $G=G_{i} H$ for all $i$. If this is the case, then for $v=g_{1} \cdots g_{r} H \rightarrow \infty$, we have $g_{i} \rightarrow \infty$ for all $i$ and Theorem 7.1 holds (see [Sh, Corollary 1.2]).

For $T>0$ and $f \in C_{c}(W)$, define

$$
F_{T}(g)=\sum_{\gamma \in \Gamma /(\Gamma \cap H)} f\left(g \gamma v_{0} / T\right), \quad g \in G / \Gamma .
$$

Proposition 7.4. Let $\phi \in C_{c}(G / \Gamma)$ such that $\int_{G / \Gamma} \phi d g=1$ and $f \in C_{c}(W \backslash\{0\})$ with $\pi(\operatorname{supp} f) \cap V^{\infty} \neq \emptyset$. Then

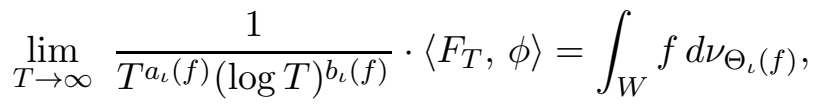

where $\nu_{\Theta_{\iota}(f)}$ is as given by Theorem 6.12. Furthermore,

$$
\lim _{T \rightarrow \infty} \int_{G / H} f\left(g v_{0} / T\right) d \mu(g)=\infty \Longrightarrow\left\langle F_{T}, \phi\right\rangle \sim \int_{G / H} f\left(g v_{0} / T\right) d \mu(g) \quad \text { as } T \rightarrow \infty .
$$

Proof. We have

$$
\begin{aligned}
\left\langle F_{T}, \phi\right\rangle & =\int_{G / \Gamma} \sum_{\gamma \in \Gamma / H \cap \Gamma} f\left(g \gamma v_{0} / T\right) \phi(g) d g=\int_{G / H \cap \Gamma} f\left(g v_{0} / T\right) \phi(g) d g \\
& =\int_{G / H} f\left(g v_{0} / T\right)\left(\int_{H / H \cap \Gamma} \phi(g h) d h\right) d \mu(g) .
\end{aligned}
$$

By Theorem 7.1, for every $\varepsilon>0$, there exists a compact set $D \subset G / H$ such that

$$
\left|\int_{H /(H \cap \Gamma)} \phi(g h) d h-1\right|<\varepsilon
$$

for $g \in G / H \backslash D$. Then

$$
\begin{aligned}
& \left|\left\langle F_{T}, \phi\right\rangle-\int_{G / H} f\left(g v_{0} / T\right) d \mu(g)\right| \\
& \quad \leq \varepsilon\left|\int_{G / H \backslash D} f\left(g v_{0} / T\right) d \mu(g)\right|+\mu(D)\|f\|_{\infty}\left(\|\phi\|_{\infty}+1\right) .
\end{aligned}
$$

The second part of the proposition now follows immediately. And the first part of the proposition follows from Theorem 6.12, 
Theorem 7.6. For every $f \in C_{c}(W \backslash\{0\})$ with $\pi(\operatorname{supp} f) \cap V^{\infty} \neq \emptyset$,

$$
\lim _{T \rightarrow \infty} \frac{1}{T^{a_{\iota}(f)}(\log T)^{b_{\iota}(f)-1}} \sum_{\gamma \in \Gamma /(\Gamma \cap H)} f\left(\gamma v_{0} / T\right)=\int_{W} f d \nu_{\Theta_{\iota}(f)},
$$

where $\nu_{\Theta_{\iota}(f)}$ is as in Theorem 6.12.

Proof. Without loss of generality, we may assume that $f \geq 0$. Given $\varepsilon>0$ there exists a compact symmetric neighborhood $\mathcal{O}_{\varepsilon}$ of $e$ in $G$ such that

$$
|f(g v)-f(v)|<\varepsilon \quad \forall g \in \mathcal{O}_{\varepsilon}, \forall v \in W,
$$

We can assume that $\mathcal{O}_{\varepsilon} \subset \mathcal{O}_{1}$. Define $f_{\varepsilon}^{ \pm} \in C_{c}(W)$ by

$$
f_{\varepsilon}^{+}(v):=\max _{g \in \mathcal{O}_{\varepsilon}} f(g \cdot v) \quad \text { and } \quad f_{\varepsilon}^{-}(v)=\min _{g \in \mathcal{O}_{\varepsilon}} f(g \cdot v), \quad \forall v \in W .
$$

For any $T>0$, let $F_{T}$ and $F_{T}^{ \pm}$be defined as in (17.3) corresponding to $f$ and $f_{\varepsilon}^{ \pm}$, respectively. Then

$$
F_{T}^{-}(g) \leq F_{T}(e) \leq F_{T}^{+}(g), \quad \forall g \in \mathcal{O}_{\varepsilon} .
$$

Hence, if $\phi \in C_{c}(G / \Gamma)$, with $\phi \geq 0, \operatorname{supp} \phi \subset \mathcal{O}_{\varepsilon}$ and $\int_{G / \Gamma} \phi d g=1$, then

$$
\left\langle F_{T}^{-}, \phi\right\rangle \leq \sum_{\gamma \in \Gamma /(\Gamma \cap H)} f\left(\gamma v_{0} / T\right)=F_{T}(e) \leq\left\langle F_{T}^{+}, \phi\right\rangle .
$$

Since each $\mathbb{R}^{+} \cdot V_{I}^{\infty}$ is $G$-invariant, we have

$$
\begin{gathered}
\Theta_{\iota}\left(f_{\varepsilon}^{+}\right)=\Theta_{\iota}(f) \supset \Theta_{\iota}\left(f_{\varepsilon}^{-}\right) \\
\left(a_{\iota}\left(f_{\varepsilon}^{+}\right), b_{\iota}\left(f_{\varepsilon}^{+}\right)\right)=\left(a_{\iota}(f), b_{\iota}(f)\right) \geq\left(a_{\iota}\left(f_{\varepsilon}^{-}\right), b_{\iota}\left(f_{\varepsilon}^{-}\right)\right) .
\end{gathered}
$$

In view of Remark 6.16, by Proposition 7.4, we get

$$
\lim _{T \rightarrow \infty} \frac{\left\langle F_{T}^{ \pm}, \phi\right\rangle}{T^{a_{\iota}(f)}(\log T)^{b_{\iota}(f)-1}}=\int_{W} f_{\varepsilon}^{ \pm} d \nu_{\Theta_{\iota}(f)} .
$$

Combining (7.8) and (7.11) we conclude that

$$
\begin{aligned}
\int_{W} f_{\varepsilon}^{-} d \nu_{\Theta_{\iota}(f)} & \leq \liminf _{T \rightarrow \infty} \frac{F_{T}(e)}{T^{a_{\iota}(f)}(\log T)^{b_{\iota}(f)-1}} \\
& \leq \limsup _{T \rightarrow \infty} \frac{F_{T}(e)}{T^{a_{\iota}(f)}(\log T)^{b_{\iota}(f)-1}} \leq \int_{W} f_{\varepsilon}^{+} d \nu_{\Theta_{\iota}(f) .}
\end{aligned}
$$

By (7.7),

$$
\int_{W} f_{\varepsilon}^{ \pm} d \nu_{\Theta_{\iota}(f)}-\int_{W} f d \nu_{\Theta_{\iota}(f)} \leq \varepsilon \cdot \nu_{\Theta_{\iota}(f)}\left(\operatorname{supp} f_{\varepsilon}^{+}\right) \leq \varepsilon \cdot \nu_{\Theta_{\iota}\left(f_{1}^{+}\right)}\left(\operatorname{supp} f_{1}^{+}\right) .
$$

By Theorem 6.12 and (17.9),$\nu_{\Theta_{\iota}\left(f_{1}^{+}\right)}\left(\operatorname{supp} f_{1}^{+}\right)<\infty$. Since $\epsilon>0$ can be chosen arbitrarily small, (7.16) follows from (17.12) and (7.14).

Note that for any $f \in C_{c}(W \backslash\{0\})$, we have $\left(a_{\iota}, b_{\iota}\right) \geq\left(a_{\iota}(f), b_{\iota}(f)\right)$. Therefore using Remark 6.16, from Theorem 7.6 and Theorem 6.4 we can deduce the following. 
Theorem 7.15. For every $f \in C_{c}(W)$,

$$
\lim _{T \rightarrow \infty} \frac{1}{T^{a_{\iota}}(\log T)^{b_{\iota}-1}} \sum_{\gamma \in \Gamma /(\Gamma \cap H)} f\left(\gamma v_{0} / T\right)=\int_{W} f d \nu_{\iota},
$$

where $\nu_{\iota}$ is as in Theorem 6.4.

Proof of Theorem 1.12, Let $\Gamma \subset G(\mathbb{Q})$ be an arithmetic subgroup that preserves the integral structure on $W(\mathbb{Z})$. Since $\mathbf{G}$ and $\mathbf{H}$ admit no nontrivial $\mathbb{Q}$-characters, by $[\mathbf{B H}]$, $\Gamma$ is an irreducible lattice in $G, \Gamma \cap H$ is a lattice in $H$, and $V(\mathbb{Z})$ is a union of finitely many orbits of $\Gamma$ :

$$
V(\mathbb{Z})=\bigcup_{i=1}^{n} \Gamma g_{i} v_{0}
$$

For any $T>0$, consider a locally finite measure $\tau_{T}$ on $W$ defined by

$$
\tau_{T}(f)=\frac{1}{T^{a_{\iota}}(\log T)^{b_{\iota}-1}} \sum_{v \in V(\mathbb{Z})} f(v / T), \quad f \in C_{c}(W) .
$$

Then

$$
\begin{aligned}
\tau_{T}(f) & =\frac{1}{T^{a_{\iota}}(\log T)^{b_{\iota}-1}} \sum_{i=1}^{n} \sum_{\gamma \in \Gamma /\left(\Gamma \cap g i H g_{i}^{-1}\right)} f\left(\gamma g_{i} v_{0} / T\right) \\
& =\frac{1}{T^{a_{\iota}}(\log T)^{b_{\iota}-1}} \sum_{i=1}^{n} \sum_{\gamma \in g_{i}^{-1} \Gamma g_{i} /\left(g_{i}^{-1} \Gamma g_{i} \cap H\right)} f\left(g_{i} \gamma v_{0} / T\right) .
\end{aligned}
$$

Note that $g_{i} \Gamma g_{i} \subset G(\mathbb{Q})$ is an arithmetic subgroup of $G$, and $\left(g_{i} \Gamma g_{i}^{-1}\right) \cap H$ is a lattice in $H$. It follows from Theorem 7.15 that the limit

$$
\tau=\lim _{T \rightarrow \infty} \tau_{T}
$$

exists in the weak* topology, and $\tau$ is the $G$-invariant measure concentrated on $G(\mathcal{W}$. $\left.v_{0}\right)^{I_{\iota}}$ which is given by

$$
\tau=\left(\sum_{i=1}^{n} \frac{\operatorname{Vol}\left(H /\left(g_{i}^{-1} \Gamma g_{i} \cap H\right)\right)}{\operatorname{Vol}(G / \Gamma)}\right) \nu_{\iota}
$$

where $\nu_{\iota}$ is as in Theorem 6.4.

Let $\phi \in C(S(W)), \phi \geq 0$, and let $\psi$ be the characteristic function of $[1 / 2,1)$. Take $c>1$, close to 1 , and $\psi^{-}, \psi^{+} \in C_{c}((1 / 4,2))$ such that

$$
0 \leq \psi^{ \pm} \leq 1, \quad \psi^{-} \leq \psi \leq \psi^{+},\left.\quad \psi^{-}\right|_{\left[c / 2, c^{-1}\right]}=1, \quad \operatorname{supp}\left(\psi^{+}\right) \subset\left[c^{-1 / 2, c] .}\right.
$$

Then for $f_{\psi}(v):=\phi(\pi(v)) \psi(\|v\|), \forall v \in W$, we have

$$
\begin{gathered}
\tau_{T}\left(f_{\psi^{-}}\right) \leq \tau_{T}\left(f_{\psi}\right) \leq \tau_{T}\left(f_{\psi^{+}}\right), \\
\tau\left(f_{\psi^{+}}\right) \leq \tau\left(f_{\psi}\left(c^{-1} v\right)\right)=c^{a_{\iota}} \tau\left(f_{\psi}\right), \\
\tau\left(f_{\psi^{-}}\right) \leq \tau\left(f_{\psi}\left(c^{-1} v\right)\right)=c^{-a_{\iota}} \tau\left(f_{\psi}\right) .
\end{gathered}
$$


Taking $c \rightarrow 1$, this implies that

$$
\lim _{T \rightarrow \infty} \frac{1}{T^{a_{\iota}}(\log T)^{b_{\iota}-1}} \sum_{v \in V(\mathbb{Z}): T / 2 \leq\|v\|<T} \phi(\pi(v))=\lim _{T \rightarrow \infty} \tau_{T}\left(f_{\psi}\right)=\tau\left(f_{\psi}\right) .
$$

Using that

$$
\#\left(V(\mathbb{Z}) \cap B_{T}\right) \ll T^{a_{\iota}}(\log T)^{b_{\iota}-1},
$$

the proof can be completed by an easy geometric series argument.

We also compute an explicit formula for the limit measure $\mu_{\iota}$. Let $\phi \in C(S(W)), \chi$ be the characteristic function of $(0,1)$, and define

$$
f_{\chi}(v)=\phi(\pi(v)) \psi(\|v\|), \quad \text { for all } v \in W .
$$

It follows from (6.8) that for some $c_{1}, c_{2}>0$,

$$
\begin{aligned}
\int_{S(W)} \phi d \mu_{\iota} & =c_{1} \int_{K} \sum_{w \in \mathcal{W}} \int_{\mathfrak{a}^{I_{\iota},+}} \int_{\mathbb{R}} f_{\chi}\left(k \exp (a) e^{t}\left(w v_{0}\right)^{I_{\iota}}\right) \xi_{I_{\iota}}(a) e^{a_{\iota} t} d t d a d k \\
& =c_{2} \int_{K} \sum_{w \in \mathcal{W}} \int_{\mathfrak{a}^{I_{\iota},+}} \phi\left(\pi\left(k \exp (a)\left(w v_{0}\right)^{I_{\iota}}\right)\right) \frac{\xi_{I_{\iota}}(a)}{\left\|k \exp (a)\left(w v_{0}\right)^{I_{\iota}}\right\|^{a_{\iota}}} d a d k .
\end{aligned}
$$

Proof of Theorem 1.11. Theorem 1.11 follows from Theorem 1.12 approximating the characteristic function of the cone by continuous functions.

Proofs of Theorems 1.18 and 1.19 . Proofs are based on Theorem 6.12 and Theorem 7.6 and are similar to the proofs of Theorem 1.11 and Theorem 1.12. We skip details. It follows from Theorem 6.12 that the measure $\mu_{\Theta_{\iota}(\phi)}$ is given by the formula

$$
\begin{aligned}
& \int_{S(W)} \phi d \mu_{\Theta_{\iota}(\phi)} \\
= & c \sum_{I \in \Theta_{\iota}(\phi)} \int_{K} \sum_{w \in \mathcal{W}} \int_{\mathfrak{a}^{I,+}} \phi\left(\pi\left(k \exp (a)\left(w v_{0}\right)^{I}\right)\right) \frac{\xi_{I}(a)}{\left\|k \exp (a)\left(w v_{0}\right)^{I}\right\|^{a_{\iota}(\phi)}} d a d k
\end{aligned}
$$

where $\phi \in C(S(W))$ and $c>0$.

7.1. Proof of Corollary 1.7. Take any $\varepsilon>0$ and Consider the cone

$$
\mathcal{C}=\left\{w \in W \backslash\{0\}:\left\|\pi(w)-v_{0}\right\|<\varepsilon\right\} .
$$

Since $V^{\infty}$ has only finitely many orbits of $G$ the cone is generic for sufficiently small $\varepsilon>0$.

Suppose $\partial \mathcal{C}$, the boundary of $\mathcal{C}$, has strictly positive measure with respect to the smooth measure class on a $G$-orbit, say $\mathcal{O}_{1}$, contained in $V^{\infty}$. Since $\mathcal{O}_{1}$ and $\partial \mathcal{C}$ are real analytic varieties, we conclude that $\mathcal{O}_{1} \subset \partial \mathcal{C}$. Since $V^{\infty}$ has only finitely many distinct $G$-orbits, and $\partial \mathcal{C}$ are disjoint for distinct $\varepsilon>0$, we conclude that $\mathcal{C}$ is admissible for sufficiently small $\varepsilon>0$. Now the corollary follows from Theorem 1.6 . 


\section{Comparison with Chambert-Loir-Tschinkel Conjecture}

Recently, Chambert-Loir and Tschinkel proposed a general conjecture about asymptotics of the number of integral points on algebraic varieties. A weaker version of this conjecture appeared in [HT]. To facilitate a comparison, we state some of our results using the language of arithmetic algebraic geometry.

Let $\mathbf{G}$ be a connected $\mathbb{Q}$-simple adjoint algebraic group, which is isotropic over $\mathbb{R}$, and $\mathbf{X}$ the wonderful compactification of $\mathbf{G}$. The wonderful compactification was constructed over $\mathbb{C}$ in $[\mathrm{CP}$ and over arbitrary field of odd characteristic in [CS]. It is a smooth projective variety defined over $\mathbb{Q}$ such that $\mathbf{G}$ is contained densely in $\mathbf{X}$, and $D:=\mathbf{X} \backslash \mathbf{G}$ is a divisor with normal crossings and smooth irreducible components. Given a field $k \supset \mathbb{Q}$, we set $\mathbf{X}_{k}=\mathbf{X} \times_{\mathbb{Q}} k$. Let $\operatorname{Pic}\left(\mathbf{X}_{\mathbb{C}}\right)$ be the absolute Picard group, $\Lambda_{\text {eff }}\left(X_{\mathbb{C}}\right) \subset \operatorname{Pic}\left(\mathbf{X}_{\mathbb{C}}\right) \otimes \mathbb{R}$ the effective cone, and $K_{\mathbf{X}}$ the canonical class. We denote by $\Delta_{\mathbb{C}}$ the system of simple roots of $\mathbf{G}$. It was shown in [CP] (cf. [BK, Sec. 6.1]) that there is an isomorphism $\lambda \mapsto\left[L_{\lambda}\right]$ between the weight lattice of $\mathbf{G}$ and the Picard group $\operatorname{Pic}\left(\mathbf{X}_{\mathbb{C}}\right)$ such that the irreducible components of the boundary divisor $D$ correspond to $L_{\alpha}, \alpha \in \Delta_{\mathbb{C}}$. Note that these irreducible components generate a finite index subgroup in $\operatorname{Pic}\left(\mathbf{X}_{\mathbb{C}}\right)$ (the root lattice). Given $v \in \mathbf{X}(\mathbb{C})$ and $[L]=\sum_{\alpha \in \Delta_{\mathbb{C}}} q_{\alpha}\left[L_{\alpha}\right] \in \operatorname{Pic}\left(\mathbf{X}_{\mathbb{C}}\right)$, we set

$$
I(v)=\left\{\alpha \in \Delta_{\mathbb{C}}: v \in \operatorname{supp} L_{\alpha}\right\} \quad \text { and } \quad[L]_{v}=\sum_{\alpha \in I(v)} q_{\alpha}\left[L_{\alpha}\right] .
$$

We define a metric on the real projective space:

$$
d([x],[y])=\frac{\|x \wedge y\|}{\|x\| \cdot\|y\|},
$$

where $\|\cdot\|$ is the standard Euclidean norm.

Theorem 8.1. Let $\mathcal{G}$ be a group scheme over $\operatorname{Spec}(\mathbb{Z})$ with generic fiber $\mathbf{G}$. Then there exists $k \in \mathbb{N}$ such that for every ample metrized line bundle $\mathcal{L}=\left(L, H_{\mathcal{L}}\right)$ on $\mathbf{X}$ defined over $\mathbb{Q}$, every $v \in(\mathbf{X} \backslash \mathbf{G})(\mathbb{R})$, and every sufficiently small $\varepsilon=\varepsilon(v)>0$,

$$
\#\left\{z \in \mathcal{G}\left(\frac{1}{k} \mathbb{Z}\right): d(z, v)<\varepsilon, H_{\mathcal{L}}(z)<T\right\} \sim_{T \rightarrow \infty} c \cdot T^{a}(\log T)^{b-1},
$$

where

$$
\begin{aligned}
& c=c(v, \mathcal{L}, \varepsilon)>0, \\
& a=a(v, L)=\inf \left\{r: r[L]_{v}+\left[K_{\mathbf{X}}+D\right]_{v} \in \Lambda_{\mathrm{eff}}\left(\mathbf{X}_{\mathbb{C}}\right)_{v}\right\}, \\
& b=b(v, L)=\left\{\begin{array}{l}
\text { the co-dimension of the face of } \Lambda_{\mathrm{eff}}\left(\mathbf{X}_{\mathbb{C}}\right)_{v} \\
\text { containing } a[L]_{v}+\left[K_{\mathbf{X}}+D\right]_{v}
\end{array}\right\} .
\end{aligned}
$$

Remark 8.2. Theorem 8.1 holds with $k=1$ if we take $v \in \overline{\mathcal{G}(\mathbb{Z}) \mathbf{G}(\mathbb{R})^{\circ}}$. In particular, we can take $k=1$ when $\mathbf{G}(\mathbb{R})=\mathcal{G}(\mathbb{Z}) \mathbf{G}(\mathbb{R})^{\circ}$. This equality holds assuming that $\mathbf{G}$ is $\mathbb{Q}$-split and $\mathcal{G}$ is the canonical $\mathbb{Z}$-model of $\mathbf{G}$ (see [GaO, Remark in Sec. 2]).

Remark 8.3. Our results also apply to non-smooth compactifications of $\mathbf{G}$ (for example, one can take the closure of the image of $\mathbf{G}$ under the irreducible representation with the highest weight $\sum_{\alpha} n_{\alpha} \omega_{\alpha}$ with some $\left.n_{\alpha}=0\right)$. We expect that an analogue 
of Theorem 8.1 holds with parameters $(a, b)$ computed with respect to the minimal resolution of singularities of the pair $(\mathbf{X}, D)$. A basic example of this type was worked out in $[\mathrm{HT}$.

Proof of Theorem 8.1. We refer to [BK, Sec. 6.1] for standard facts about the wonderful compactification. Recall that the effective cone $\Lambda_{\mathrm{eff}}\left(\mathbf{X}_{\mathbb{C}}\right)$ is generated by $\left[L_{\alpha}\right]$ for $\alpha \in$ $\Delta_{\mathbb{C}}$, the ample cone is generated by $\left[L_{\omega_{\alpha}}\right]$ for $\alpha \in \Delta_{\mathbb{C}}\left(\omega_{\alpha}\right.$ 's are the fundamental weights), and

$$
K_{\mathbf{X}} \sim-L_{2 \rho_{\mathbb{C}}}-\sum_{\alpha \in \Delta_{\mathbb{C}}} L_{\alpha}
$$

where $2 \rho_{\mathbb{C}}$ is the sum of positive roots of $\Delta_{\mathbb{C}}$. The support of $L_{\alpha}$ is isomorphic to the fibration over $\mathbf{G} / \mathbf{P}_{\alpha} \times \mathbf{G} / \mathbf{P}_{\alpha}$, where $\mathbf{P}_{\alpha}$ is the maximal parabolic subgroup corresponding to $\alpha$, with fibers equal to the wonderful compactification of the adjoint form of the Levi subgroup of $\mathbf{P}_{\alpha}$. This implies that the Galois action on $\operatorname{Pic}\left(\mathbf{X}_{\mathbb{C}}\right)$ correspond to the twisted Galois action ( $\star$-action) on $\Delta_{\mathbb{C}}$.

We denote by $\Delta$ the system of restricted roots (with respect to a Cartan involution) chosen so that $r\left(\Delta_{\mathbb{C}}\right)=\Delta \cup\{0\}$ where $r$ is the restriction map. We have the decomposition into disjoint $\mathbf{G}$-orbits:

$$
\mathbf{X}=\bigcup_{I \subset \Delta_{\mathbb{C}}} \mathbf{O}_{I}
$$

where $\mathbf{O}_{\Delta_{\mathbb{C}}}=\mathbf{G}$ and $\mathbf{O}_{I} \subset \operatorname{supp} L_{\alpha}$ iff $\alpha \notin I$. The structure of the set $\mathbf{X}(\mathbb{R})$ was described in [BJ, Ch. 7]. In particular, we have

$$
\mathbf{X}(\mathbb{R})=\bigcup_{I \subset \Delta} \mathbf{O}_{r^{-1}(I)}(\mathbb{R}) .
$$

The set $\mathbf{X}(\mathbb{R})$ is a union of of finitely many Satake compactifications $\bar{V}$ of $\mathbf{G}(\mathbb{R})^{o}$ so that $V_{I}^{\infty} \subset \mathbf{O}_{r^{-1}(I)}(\mathbb{R})$ for every $I \subset \Delta$. It follows from the weak approximation for $\mathbf{G}$ that each connected component of $\mathbf{G}(\mathbb{R})$ contains a rational point. We take $k \in \mathbb{N}$ so that each connected component of $\mathbf{G}(\mathbb{R})$ contains a point from $\mathcal{G}\left(\frac{1}{k} \mathbb{Z}\right)$. By BorelHarish-Chandra theorem, $\mathcal{G}\left(\frac{1}{k} \mathbb{Z}\right)$ is a union of finitely many $\mathcal{G}(\mathbb{Z})$-orbits, and it suffices to compute the asymptotic for each of these orbits. For simplicity, we consider the orbit of the identity.

For $\alpha \in \Delta$, we set

$$
L_{\alpha}:=\sum_{\beta \in \Delta_{\mathbb{C}}} L_{r^{-1}(\alpha)}
$$

Let $J=r(I(v)) \subset \Delta$. Then $v \in V_{\Delta \backslash J}^{\infty}$. If $L \sim L_{\lambda}$ for a dominant weight $\lambda$, we get

$$
[L]_{v}=\sum_{\alpha \in J} \frac{m_{\alpha}}{\# r^{-1}(\alpha)} \cdot\left[L_{\alpha}\right], \quad\left[K_{\mathbf{X}}+D\right]_{v}=\sum_{\alpha \in J} \frac{u_{\alpha}}{\# r^{-1}(\alpha)} \cdot\left[L_{\alpha}\right],
$$

where $m_{\alpha}$ 's and $u_{\alpha}$ 's are given as in (1.9). 
Passing to a tensor power, we may assume that $L \sim L_{\lambda}$ is very ample and linearized, and $\lambda$ is in the root lattice. Let $\iota$ be the $\mathbb{Q}$-representation of $\mathbf{G}$ on $H^{0}(\mathbf{X}, L)$. Then

$$
H_{\mathcal{L}}(z)=H(\iota(z)), \quad z \in \mathcal{G}(\mathbb{Z}),
$$

where $H$ is the standard height function with respect to a lattice $\Lambda \subset H^{0}(\mathbf{X}, L)$. Passing to a finite index subgroup, if necessary, we may assume that $\iota(\mathcal{G}(\mathbb{Z})) \subset \operatorname{Stab}(\Lambda)$. Then

$$
H_{\mathcal{L}}(z)=\|\iota(z)\|, \quad z \in \mathcal{G}(\mathbb{Z}),
$$

where $\|\cdot\|$ is a norm on $H^{0}(\mathbf{X}, L) \otimes \mathbb{R}$. Since the representation $\iota$ has the unique highest weight $\lambda$, the results of Section 1 apply (see Corollary 1.7 and Example 2.4).

\section{REFERENCES}

[BM] V. Batyrev and Y. Manin Sur le nombre des points rationnels de hauteur borné des variétés algébriques, Math. Ann. 286, 1990, 27-43.

[BT] V. Batyrev and Y. Tschinkel Tamagawa numbers of polarized algebraic varieties. Nombre et répartition de points de hauteur bornée (Paris, 1996), Astérisque 251, 1998, 299-340.

[BH] A. Borel and Harish-Chandra Arithmetic subgroups of Algebraic groups, Ann. Math. 75, 1962, 485-535.

[BJ] A. Borel and L. Ji Compactifications of symmetric and locally symmetric spaces, Birkhaüser, Inc., Boston, MA, 2005.

[BK] M. Brion and S. Kumar Frobenius splitting methods in geometry and representation theory, Birkhaüser Inc., Boston, MA, 2005.

[CP] C. De Concini and C. Procesi Complete symmetric varieties. Invariant theory (Montecatini, 1982), 1-44, Lecture Notes in Math., 996, Springer, Berlin, 1983.

[CS] C. De Concini and T. Springer Compactification of symmetric varieties, Transform. Groups 4, 1999, 273-300.

[DM1] S. G. Dani and G. Margulis Asymptotic behavior of trajectories of unipotent flows on homogeneous spaces, Proc. Indian. Acad. Sci. (Math. Sci), Vol 101, No 1, 1991, pp. 1-17.

[DM2] S. Dani, G. Margulis Limit distribution of orbits of unipotent flows and values of quadratic forms, Advances in Soviet Math., 16 (1993) p. 91-137

[DRS] W. Duke, Z. Rudnick and P. Sarnak Density of integer points on affine homogeneous varieties, Duke Math. J. 71, 1993, 181-209.

[EM] A. Eskin and C. McMullen Mixing, counting and equidistribution in Lie groups, Duke Math. J. 71, 1993, 143-180.

[EMS] A. Eskin, S. Mozes, N. Shah Unipotent flows and counting lattice points on homogeneous varieties, Annals of Math. 143 (1996) p.149-159.

[FMT] J. Franke, Y. Manin, Y. Tschinkel Rational points of bounded height on Fano varieties, Invent. Math. 95, 1989, 421-435.

[HT] B. Hassett and Y. Tschinkel Integral points and effective cones of moduli spaces of stable maps, Duke Math. J. 120, 2003, 577-599 (correction: Duke Math. J. 125, 2004, 205-206).

[HS] G. Heckman and H. Schlichtkrull Harmonic analysis and special functions on symmetric spaces, Perspectives in Mathematics, 16. Academic Press, Inc., 1994.

[GaO] W. T. Gan and H. Oh Equidistribution of integer points on a family of homogeneous varieties: a problem of Linnik, Compositio Math. 136, 2003, 325-352.

[GMO] A. Gorodnik, F. Maucourant, H. Oh Manin's and Peyre's conjectures on rational points and adelic mixing, Preprint (2005).

[GO] A. Gorodnik and H. Oh Orbits of discrete subgroups on a symmetric space and Furstenberg boundary, Duke Math. J., Vol 139, 2007, 483-525

[GW] A. Gorodnik and B. Weiss Distribution of lattice orbits on homogeneous varieties, GAFA., Vol 17,2007 
[GJT] Y. Guivarc'h, L. Ji, J. C. Taylor Compactifications of symmetric spaces, Progress in Mathematics, 156. Birkhaüser Boston, Inc., Boston, MA, 1998.

[M] F. Maucourant Homogeneous asymptotic limits of Haar measures of semisimple linear groups and their lattices, Duke Math. J., Vol 136, 2007, 357-399

[OV] A. L. Onishchik and E. B. Vinberg Lie groups and Lie algebras III: Structure of Lie groups and Lie algebras, Encyclopaedia of Mathematical Sciences, 41. Springer-Verlag, Berlin, 1994.

[On] A. Onishchik Lectures on real semisimple Lie algebras and their representations, ESI Lectures in Mathematics and Physics. European Mathematical Society (EMS), Zürich, 2004.

[Os] T. Oshima A realization of semisimple symmetric spaces and construction of boundary value maps, Adv. Studies in Pure Math. vol 14, Representations of Lie groups, 1986, pp. 603-650.

[OS] T. Oshima and J. Sekiguchi The restricted root system of a semisimple symmetric pair, Group representations and systems of differential equations (Tokyo, 1982), 433-497, Adv. Stud. Pure Math., 4, North-Holland, Amsterdam, 1984.

[P] E. Peyre Hauteurs et mesures de Tamagawa sur les variétés de Fano, Duke Math. J. 79, 1995, $101-218$.

[Ra] M. Ratner On Raghunathan's measure conjecture, Ann. of Math., Vol 134, 1991, pp. 545-607.

[Ro] W. Rossmann The structure of semisimple symmetric spaces, Canad. J. Math. 31, 1979, 157-180.

[Sa] I. Satake On representations and compactifications of symmetric Riemannian spaces, Ann. Math. 71, 1960, 77-110.

[Sc] H. Schlichtkrull Hyperfunctions and Harmonic Analysis on Symmetric Spaces, Progress in Mathematics, 49. Birkhaüser Boston, Inc., Boston, MA, 1984.

[Sh] N. Shah Limit distribution of expanding translates of certain orbits on homogeneous spaces, Proc. Indian. Acad. Sci. Math. 58, 2001, 461-471.

[STT] J. Shalika, R. Takloo-Bighash, Y. Tschinkel Rational points on compactifications of semisimple groups, Preprint (2005).

[T] J. Tits Représentations linéaires irréductibles d'un groupe réductif sur un corps quelconque, J. Reine Angew. Math. 247, 1971, 196-220.

[Ts] Y. Tschinkel Geometry over non-closed fields, ICM lecture, 2006

Mathematics 253-37, Caltech, Pasadena, CA 91106

E-mail address: gorodnik@caltech.edu

Mathematics 253-37, Caltech, Pasadena, CA 91106

Current address: Math Department, 151 Thayer St., Brown University, Providence, RI 02912

E-mail address: heeoh@math.brown.edu

School of Mathematics, TifR, 1 Homi Bhabha Road, Mumbai, 400005, India

E-mail address: nimish@math.tifr.res.in 